\title{
Quantitative Analyse von Korallengemeinschaften des Sanganeb-Atolls (mittleres Rotes Meer). I. Die Besiedlungsstruktur hydrodynamisch unterschiedlich exponierter Außen- und Innenriffe
}

\author{
H. Mergner ${ }^{1}$ \& H. Schuhmacher ${ }^{2}$ \\ ${ }^{1}$ Ruhr-Universität Bochum, Lehrstuhl für Spezielle Zoologie; \\ Postfach 1021 48, D-4630 Bochum, Bundesrepublik Deutschland \\ 2 Universität GHS Essen, Lehrstuhl für Hydrobiologie; \\ Postfach 1037 64, D-4300 Essen, Bundesrepublik Deutschland
}

\begin{abstract}
Quantitative analysis of coral communities of Sanganeb-Atoll (central Red Sea). I. The community structure of outer and inner reefs exposed to different hydrodynamic regimes. The Sanganeb-Atoll off Port Sudan is an elongate annular reef which rests on a probably raised block in the fracture zone along the Red Sea-graben. Its gross morphology was most likely formed by subaerial erosion during low sealevel conditions. Features of its topography and hydrography are described. The prevailing wind waves are from NE. Hence, the outer and inner reef slopes are exposed to different hydrodynamic conditions. The sessile benthos was analysed using the quadrat method. Four test quadrats $(5 \times 5 \mathrm{~m}$ each) were selected on the outer and inner slopes at a depth of $10 \mathrm{~m}$ along a SSW-NNE transect across the atoll. Cnidaria were by far the most dominating group; coralline algae, Porifera, Bryozoa and Ascidia, however, counted for just under $3 \%$ living cover. Light and temperature intensities did not differ significantly at the sites studied; water movement, however, decreased in the following order: TQ IV (outer NE side of the reef ring) was exposed to strong swell and surf; TQ II (inner side of the SW-ring) was met by a strong longreef current; TQ I was situated on the outer lee of the SW-atoll ring and TQ III in the inner lee of the NE-side. This hydrodynamic gradient correlates with the composition of the coral communities from predominantly branching Scleractinia (staghorn-like and other Acropora species and Pocillopora) in TQ IV, through a Lobophyllia, Porites and Xenia-dominated community in TQ $\mathrm{II}_{\text {r }}$ and a mixed community with an increasing percentage of xeniid and alcyoniid soft corals in TQ $I$, to a community in TQ III which is dominated by the soft corals Sinularia and Dendronephthya. The cnidarian cover ranged between 42.4 and $56.6 \%$ whereby the two exposed test quadrats had a higher living coverage than the protected ones. In total, 2649 colonies comprising 124 species of stony, soft and hydrocorais were recorded by an elaborate method of accurate in-situ mapping. The 90 scleractinian species found include 3 species new to the Red Sea and 11 hitherto unknown species from its central part. Only 7 cnidarian species covered large areas, forming a Xenia macrospiculata-zone in TQ I, a Lobophyllia corymbosa-zone in TQ II, a Sinularia-Dendronephthya-zone in TQ III and an Acropora-Pocillopora verrucosa-zone in TQ IV. Hydrodynamic exposition and ratio of genus abundance Acropora:Montipora are closely correlated: on the windward side (TQ IV) it is $943: 4$, but on the leeward side (TQ I) 18:208. Apart from the taxonomic and quantitative distribution of species and colonies, types of growth form and categories of the dead substrate are given. Since soft corals do not contribute to the reef framework, the distribution of Scleractinia and Alcyonaria indicates that at Sanganeb-Atoll reef substance is mainly generated on the windward side.
\end{abstract}




\section{EINLEITUNG}

Das Atoll ist von anderen Rifftypen dadurch unterschieden, daß das Riff ringförmig eine Lagune umschließt (Darwin, 1842) und keinerlei Uberwasseranteile des zugrunde liegenden Felssockels aufweist (u. a. Cloud, 1957; McNeil, 1972). Falls Inseln den Wasserspiegel überragen, bestehen sie ausschließlich aus biogenem Riffsediment. Im Gegensatz zum Saumriff, welches Landmassen unmittelbar vorgelagert ist, und auch zum Barriereriff, das sich meist noch im Bereich von Landeinflüssen befindet, bleibt also das Atoll von terrigenen Einwirkungen unberührt. Hochwasserschübe und Schlammzufuhr aus Flußmündungen, gelegentliche oder chronische Landabschwemmungen und andere durch Küstennähe bedingte Faktoren können beim Atoll unberücksichtigt bleiben, wenn die maßgeblichen Umwelteinflüsse für seine Riffgemeinschaften analysiert werden sollen.

Wie in zahlreichen Untersuchungen nachgewiesen, spielen Lage und Ausrichtung eines Riffabschnittes zur vorherrschenden Wasserbewegung eine herausragende Rolle für seine Besiedlung (z. B. Wood-Jones, 1910; Marshall, 1931; Hiatt, 1957; Mergner, 1985; speziell für das Rote Meer: Mergner, 1967; Mergner \& Schuhmacher, 1974). Ein Atoll bietet mit seinen vier unterschiedlich exponierten Riffseiten die Möglichkeit, den Einfluß der Wasserbewegung vergleichend zu studieren. Qualitative Untersuchungen zur Besiedlung der luv- und leeseitigen Riffpartien finden sich daher auch schon unter frühen Riffzonierungsarbeiten (vgl. Wells, 1957; Stoddart, 1969). Sie bezogen sich freilich fast ausnahmslos auf das Riffdach. Nur Wells (1954) schloß in seiner klassischen Arbeit über das Bikini-Atoll auch den Riffaußenhang (inkl. Vorriff) sowie die Lagunenseite mit ein. Ausgenommen blieb lediglich der obere Riffhang unterhalb der Riffaußenkante. Die hier fast immer starke Brandung machte bei Bikini - wie auch an anderen ozeanischen Riffen - diesen Riffabschnitt einer näheren Untersuchung unzugänglich, weshalb er von Wells auch als "mare incognita" bezeichnet wurde.

Die vorliegende Arbeit hat sich zum Ziel gesetzt, gerade diesen wenig bekannten Riffabschnitt hinsichtlich seiner Besiedlungsstrukturen - je nach Lage am Außen- und Innenhang sowie seiner Orientierung zur Luv- und Leeseite - an exemplarischen Ausschnitten quantitativ zu analysieren. Dabei ist noch folgende Überlegung für die Auswahl der Tiefenstufe von etwa $10 \mathrm{~m}$ bedeutsam: Die Besiedlungsabfolgen bisheriger Transektuntersuchungen über das Riffdach lassen sich zwar gut mit einem Gradienten der Wasserbewegung korrellieren; sie sind gleichzeitig aber auch täglichen und saisonalen Temperatur- und Salinitätsschwankungen unterworfen und können außerdem durch gelegentliches Trockenfallen bzw. Aussüßen durch Regenfälle erheblich beeinflußt werden. Kurzum, der Einfluß der Wasserbewegung kann durch unvorhersehbare andere abiotische Einflüsse überlagert und somit nicht mehr eindeutig erkennbar sein. In einer Tiefe von $10 \mathrm{~m}$ ist hingegen die Wahrscheinlichkeit dafür wesentlich geringer; hier darf angenommen werden, daß vorgefundene Besiedlungsstrukturen das langfristig herrschende Wirkungsgefüge aus abiotischen Faktoren, wie Beleuchtung und Wasserbewegung, sowie biotischen Parametern widerspiegeln.

Als zu Europa nächstgelegenes Atoll wurde das Sanganeb-Atoll nordostwärts von Port Sudan für die quantitative Analyse der Besiedlungsstruktur ausgewählt. Damit wird gleichzeitig der Versuch unternommen, zur Kenntnis dieses einzigen Atolls im Roten Meer und seiner Korallengemeinschaften beizutragen.

Die ersten riffbiologischen Untersuchungen vor der sudanesischen Küste wurden 
von Crossland (1907, 1911, 1913) durchgeführt. Mergner (1967) gibt eine Einführung in die topographischen, klimatischen und hydrographischen Verhältnisse dieser Riffregion, während sich eine orientierende Ubersicht über Morphologie und Besiedlung ihrer Riffe durch Korallen und Fische bei Vine \& Vine (1980) findet. Scheer (1964) und Kühlmann (1983) berichten über Korallenaufsammlungen insbesondere vom WingateBarriereriff, und Head (1980) untersucht detailliert die Korallenassoziationen im North Towartit-Bereich. Weitere Untersuchungen aus der sudanesischen Region werden bei den Darstellungen der riffbiologischen Erforschung des Roten Meeres durch Scheer \& Pillai (1983), Mergner (1984), und Scheer (1984) genannt. Ubber einen Vergleich der Korallenbesiedlung in den Sanganeb-Arealen mit der eines Vorriffareals bei Aqaba berichtet der II. Teil der Gesamtarbeit (Schuhmacher \& Mergner, 1985).

\section{UNTERSUCHUNGSREGION UND NATURVERHÄLTNISSE}

Das Rote Meer ist, zusammen mit dem Golf von Suez, $2270 \mathrm{~km}$ lang und maximal 350 $\mathrm{km}$ breit. Seine größte Tiefe liegt bei $2605 \mathrm{~m}$, nach Wong \& Degens (1980) bei $2920 \mathrm{~m}$, und nach Nawab (1984) bei $2850 \mathrm{~m}$. Es ist ein trogartiger Graben mit einem medianen Spaltenbruch (Rift), der sich durch Auseinanderweichen der präkambrischen afrikanischen und arabischen Platten ständig verbreitert. Dabei führt "sea-floor-spreading" zur Bildung junger ozeanischer Kruste; das Rote Meer stellt somit einen Ozean " in statu nascendi" dar (u. a. Krenkel, 1925; Gohar, 1954; Girdler, 1969; Schilling, 1969; zusammenfassende Ubersicht bei Wong \& Degens, 1980).

Der Schelfsockel des Roten Meeres, besonders seine ausgedehnten Flachwassergebiete vor der sudanesischen Küste, haben die Entwicklung und Vielfalt üppiger Korallenriffe begünstigt. Dieses Schelfgebiet ist seit Entstehung des arabisch-ostafrikanischen Bruchgrabensystems und den anschließenden, o. g. Bewegungen der Erdkruste großen tektonischen Spannungen und Verschiebungen ausgesetzt. Dabei wurden offensichtlich Teile des Schelfsockels durch küstenparallele Verwerfungen und querlaufende Klüfte in Schollen zerlegt. Sie wurden teilweise als Horste emporgehoben oder in Senken hinabgedrückt. Anders lassen sich die blockartigen Horste und Rippen, Mulden und Schluchten nicht erklären, die mit fast senkrechten Wänden aneinander grenzen und - sofern sie vor die heutige Schelfkante versetzt sind - aus Tiefen von oft $800 \mathrm{~m}$ unvermittelt bis zur Wasseroberfläche hochragen (Abb. 1). Von Korallenriffen überwachsen, stellen sie sich heute als atollartige Riffe (Sanganeb-Atoll), als mächtige Plattformriffe (Shaab Rumi, Shaab Anber) oder als annähernd küstenparallele Barriereriffe (Wingate-Riff, North Towartit-Riff) dar. Detaillierte geomorphologische Untersuchungen stehen noch aus; doch weisen Brandungskehlen und Abrasionsterrassen darauf hin, daß spätere epirogenetische Bewegungen des Untergrundes sowie eustatische Meeresspiegelschwankungen den Korallenüberzug und Kalkaufbau entsprechend begrenzt oder erweitert haben. Die wenigen geomorphologischen Arbeiten aus diesem Raum werden von Braithwaite (1982) resumiert.

\section{Das Sanganeb-Atoll}

Das Sanganeb-Atoll liegt mit $19^{\circ} 45^{\prime} \mathrm{N}$ und $37^{\circ} 26^{\prime} 0$ etwa $23 \mathrm{~km}$ östlich der sudanesischen Küste, $28 \mathrm{~km}$ nordöstlich der Hafenstadt Port Sudan (Abb. 1). Sein Sockel steigt 

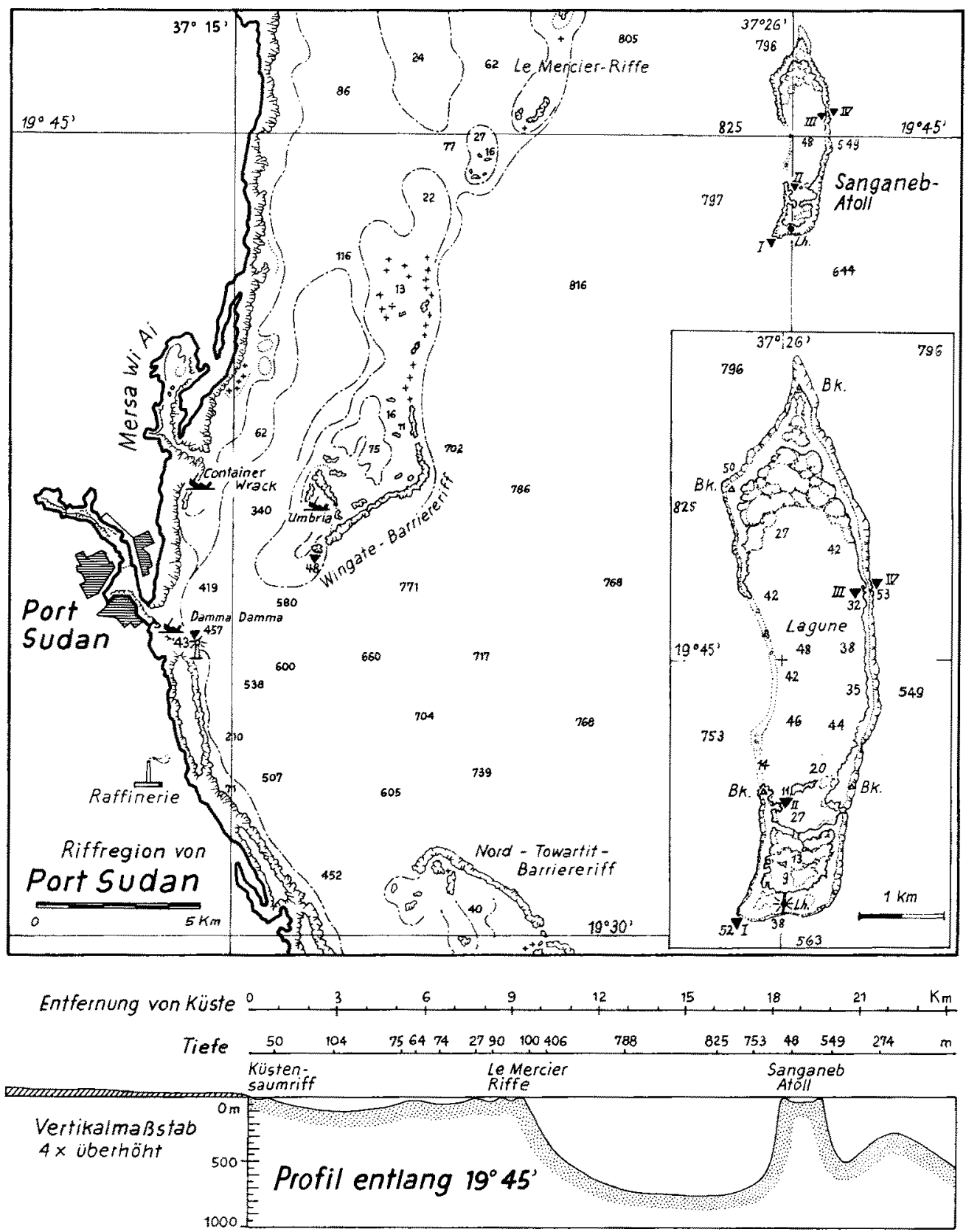

Abb. 1. Karte der Riffregion von Port Sudan. Darunter Profil entlang $19^{\circ} 45^{\prime}$ Nord von der Küste zum Sanganeb-Atoll. Nebenkarte nach Luftbild Abbildung 2 mit den Testarealen I-IV des Sanganeb- 
aus Tiefen von $550-750 \mathrm{~m}$ mit annähernd $60^{\circ}$ Neigung, auf den letzten $20-25 \mathrm{~m}$ mit etwa $80^{\circ}$ bis zur Riffkrone auf. Offensichtlich gründet sich das Riff auf einen einzelstehenden, vor die Schelfkante versetzten Horst. Der Rifftyp Atoll ist definiert durch die eingangs genannten morphologischen Kriterien - seine Genese bleibt hiervon unberührt. Frühere Versuche, den Begriff Atoll nur auf Ringriffe, die nach Darwins Subsidence-Theorie (1842) entstanden sind, anzuwenden (z. B. durch Molengraaff, 1930), haben sich nicht durchgesetzt. Eine evtl. die Atollform beeinflussende Konfiguration des Untergrundes ("Antecedent Karst Theory"; Purdy, 1974) müßte im Falle des Sanganeb-Riffs noch durch Bohrungen erhärtet werden.

Das lang-oval geformte Riff erstreckt sich in nordsüdlicher Richtung über $6 \mathrm{~km}$, in ostwestlicher Richtung maximal über $2 \mathrm{~km}$. Vor allem im Osten und Westen ist der Riffring nur knapp $100 \mathrm{~m}$ breit, während er sich im Süden auf nahezu $300 \mathrm{~m}$ verbreitert (Abb. 1 und 2). Neben einer breiten schiffbaren Passage auf der Westseite führen nur wenige flache Kanäle über das Riffdach, das an keiner Stelle die Wasseroberfläche überragt. Der Verlauf des Riffes verrät sich jedoch, von der offenen Westpassage abgesehen, überall durch die weiße Gischtlinie des Brandungssaumes (Abb. 2). Zur Lagune hin fällt das Riff zunächst unvermittelt über steile Innenränder und dann allmählich über sandigen Untergrund bis auf $48 \mathrm{~m}$ Tiefe ab. Von der Lagune wird durch eine schmale Korallenriffrippe nach Süden hin eine kleine flachere Wanne abgetrennt, die durch einen Bootskanal mit dem nördlichen tiefen Basin verbunden ist.

Der $38 \mathrm{~m}$ hohe Leuchtturm erhebt sich von der Mitte des südlichen breiten Riffdaches (Abb. 3a). Er ist von einer ca. $40 \times 40 \mathrm{~m}$ großen Betonplattform umgeben, von der aus je ein Bootssteg nach Norden zur Lagune und nach Süden zum offenen Meer führt. Auf der Plattform befindet sich die kleine Außenstelle des Institute of Oceanography, Port Sudan, von der aus die Untersuchungen durchgeführt wurden (15. Februar 15. März 1980).

\section{Klima, Wetter und hydrographische Faktoren}

Nach Angaben im Handbuch für das Rote Meer und den Golf von Aden Nr. 2034 (1963) und bei Mergner (1967) liegt die Mittelregion des Roten Meeres im Übergangsbereich zwischen den recht beständigen NW-Winden des nördlichen Meeresteiles und den jahreszeitlich regelmäßig wechselnden Monsunwinden des Südteils, d. h. im Nordwinter dem über der Straße von Bab el Mandeb auf SO abgelenkten NO-Monsun. Die mittleren Windstärken für Februar und März mit 3,2 Bft wurden während des Untersuchungszeitraumes mit durchschnittlich 4,27 Bft deutlich übertroffen. Am SanganebAtoll kam der Wind von Mitte Februar bis Mitte März 1980 aus nördlichen Richtungen (96\% der Beobachtungen) und wechselte nur kurzfristig, wie im langjährigen Mittel, auf SO. Diese allgemeinen Luftbewegungen werden von täglich regelmäßig wechselnden nächtlichen Landwinden aus N bis NW (Nordwinter) und tagsüber von kräftigen Seebrisen aus NO bis $O$ überlagert. Sie alle nehmen einen entscheidenden Einfluß auf die Bewegungen des Oberflächenwassers. Außer den durch Monsune verursachten, großräumigen Bewegungen des Oberflächenwassers zieht ganzjährig eine beständige, annähernd aus SO kommende Oberflächenströmung gegen Port Sudan. Daneben setzen andere starke, aber mehr lokale Strömungen mit Geschwindigkeiten von 0,75 bis $1 \mathrm{sm} \cdot \mathrm{h}^{-1}$ nahe dem Sanganeb-Atoll im Winter süd- und ostwärts, im Sommer in 


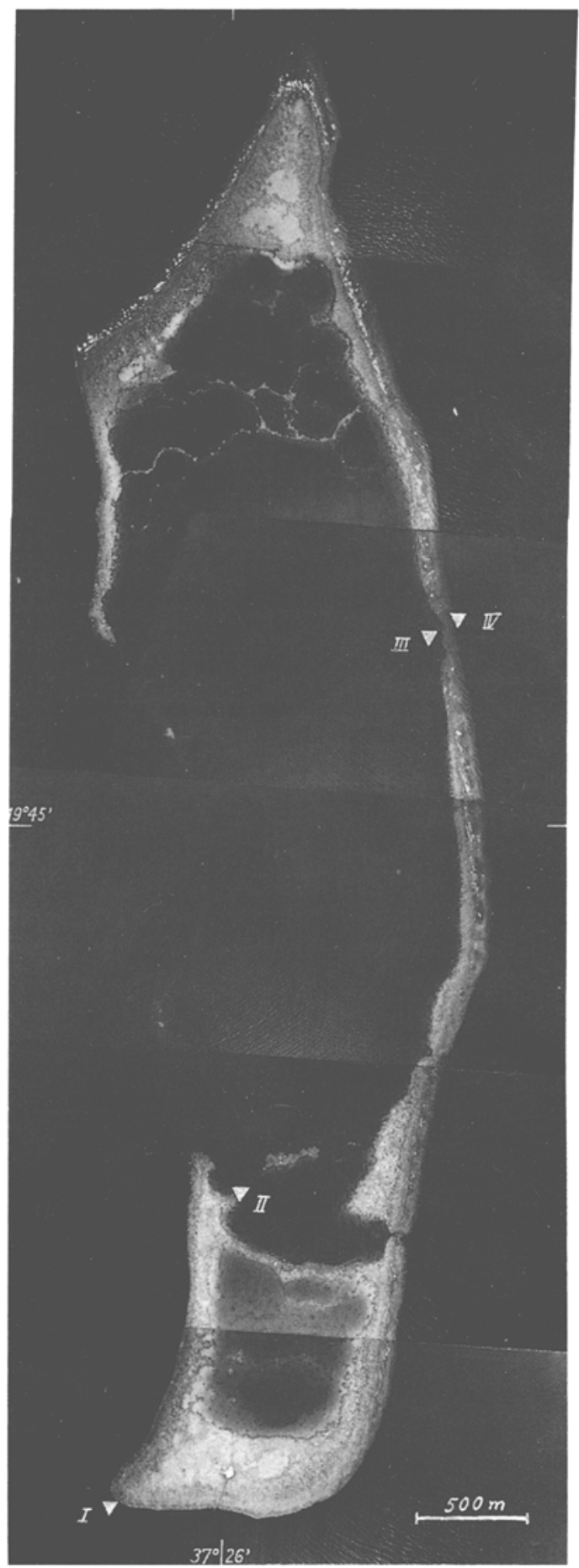

Abb. 2. Luftbild des Sanganeb-Atolls, aus den Einzelaufnahmen Nr. S38, 152 bis 157, der Serie SAgII 2003 88,33 zusammengesetzt. Veröffentlichung mit Genehmigung des Survey Department, Khartoum (Aufnahmen aus $5500 \mathrm{ft}$. [ $1676 \mathrm{~m}$ ] Höhe). I-IV Testareale 

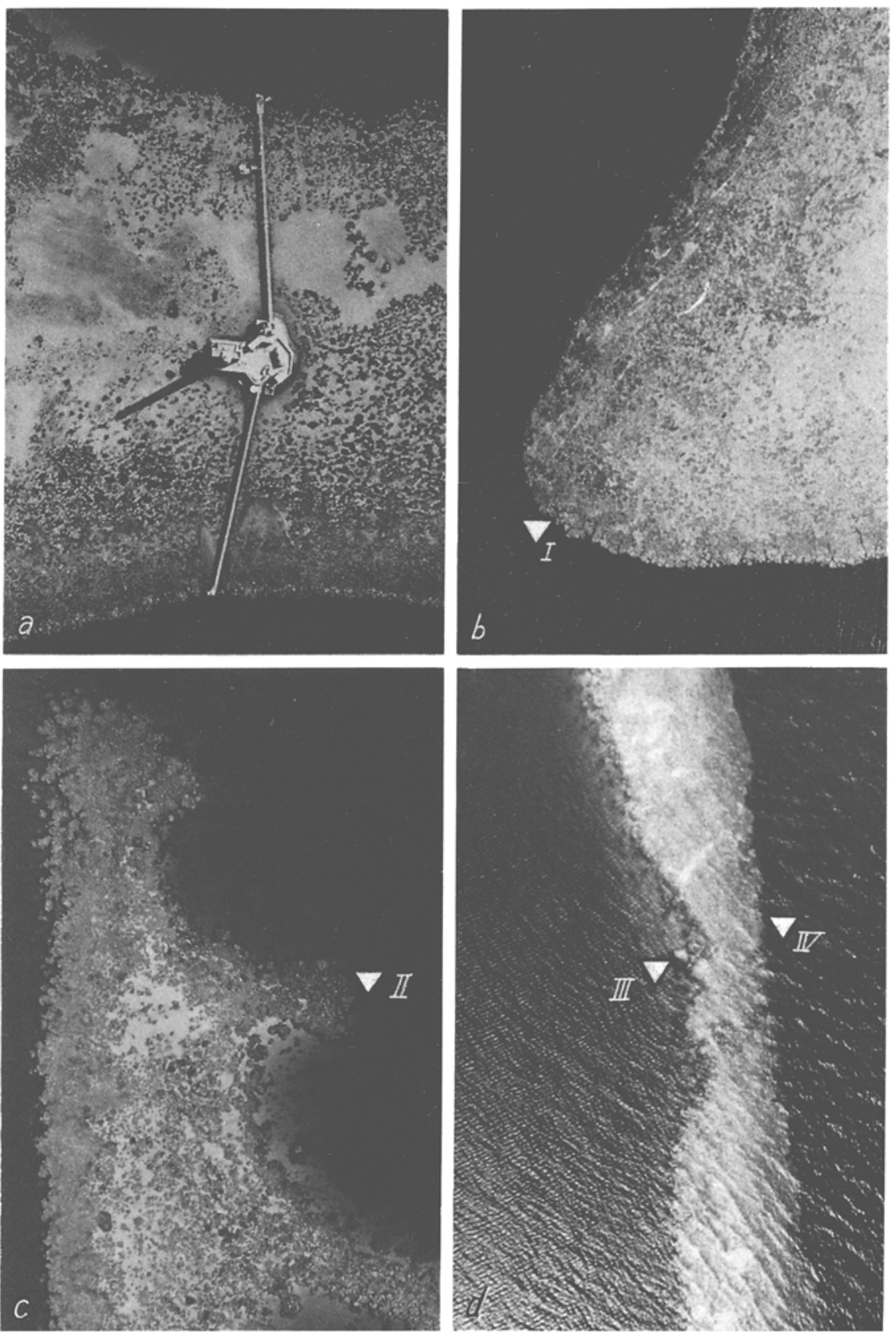

Abb. 3. Einzelluftbilder vom Sanganeb-Atoll mit Lage der Testareale I-IV. Aufnahmeserie SAgll 2003, 88,33; Einzelaufnahmen Nr. S38, 164 und 182 sowie V37, 62, 76 und 80 (Aufnahmen aus $2000 \mathrm{ft}$. [610 m] Höhe). (a) Südliche Riffplattform mit Leuchtturm, Anlegestegen und Außenstelle des Institute of Oceanography, Port Sudan. (b) SW-Ecke des Atolls mit Testareal I am Außenriff. (c) Westriff südlich der Westpassage (oben) mit Testareal II am Innenriff. (d) NO-Riff mit Testareal III am Innenriff und IV am AuBenriff 
umgekehrte Richtung. Beide jahreszeitlichen Varianten drehen dann beim WingateBarriereriff landeinwärts. Dazu verläuft im März eine starke küstenparallele Strömung zwischen Port Sudan und Mersa Wi Ai nordwärts, die für die Entwicklung der dort liegenden Saumriffe von Bedeutung sein dürfte.

Während der Bearbeitung der einzelnen Testareale am Sanganeb-Atoll ergaben sich aus Messungen und Schätzungen die in Tabelle 1 angegebenen Mittelwerte für

Tab. 1. Mittelwerte von Wind, Bewölkung und Oberflächenwasser an den vier Testarealen des Sanganeb-Atolls (16. Febr. -13, März 1980)

\begin{tabular}{|c|c|c|c|c|c|c|c|c|c|}
\hline $\begin{array}{l}\text { TQ- } \\
\text { Nr. }\end{array}$ & $\begin{array}{l}\text { Wir } \\
\text { Richtung } \\
\text { aus }\end{array}$ & $\begin{array}{l}\text { Ge- } \\
\text { schwin- } \\
\text { digkeit } \\
\text { (Bft) }\end{array}$ & $\begin{array}{c}\text { Bewöl- } \\
\text { kung } \\
(\% / 10)\end{array}$ & $\begin{array}{l}\text { Richtung } \\
\text { nach }\end{array}$ & $\begin{array}{l}\text { Obe } \\
\text { Geschw. } \\
\left(\mathrm{cm} \cdot \mathrm{s}^{-1}\right)\end{array}$ & $\begin{array}{l}\text { rf l ä ch } \\
\text { Seegang } \\
\text { (m) }\end{array}$ & $\begin{array}{l}\text { nwas } \\
\text { Bran- } \\
\text { dung }\end{array}$ & $\begin{array}{l}\text { Ur } \\
\text { Uicht } \\
\text { (m) }\end{array}$ & $\begin{array}{l}\text { Tempe- } \\
\text { ratur } \\
\left({ }^{\circ} \mathrm{C}\right)\end{array}$ \\
\hline I & NNW-N & 4,3 & 1,2 & SSO-O & $10-14$ & 0.65 & 2 & 26,3 & 25,6 \\
\hline II & $\overline{\mathrm{NNW}}-\mathrm{N}$ & 5,6 & 1,4 & $\mathrm{SO}$ & $10-14$ & 1,05 & 3 & 9,2 & 25,3 \\
\hline III & NNW- $\bar{N}$ & 3,7 & 0,3 & SO-SSO & $5-9$ & 1,04 & 3 & 17,9 & 25,5 \\
\hline IV & $\bar{N}$ & 2,7 & 2,6 & N-SO & $10-14$ & 1,11 & 3 & 31,7 & 26,0 \\
\hline
\end{tabular}

Wind, Bewölkung und Oberflächenwasser. Es ist dabei zu berücksichtigen, daß das Testquadrat IV an der brandungsexponierten NO-Seite des Atolls nur bei relativ ruhigem Seegang bearbeitet werden konnte. Selbst an diesen Tagen wurde die Seegangshöhe noch auf reichlich $1 \mathrm{~m}$ geschätzt. Fast während der Hälfte des Aufenthaltes waren die Wellen jedoch höher und verwehrten den Zugang mit dem Boot. Während eines kurzen Inspektionstauchganges bei fast sturmartigem Wind und ca. 2,5 $\mathrm{m}$ Seegangshöhe wurde $u$. a. beobachtet, wie noch in $10 \mathrm{~m}$ Tiefe eine $30 \mathrm{~cm}$ lange Fungia echinata regelmäßig von den Oszillationsbewegungen der anlaufenden Wellen umgewendet wurde.

Die Lichtverhältnisse wurden unmittelbar an den Testquadraten jeweils zur Mittagszeit bei wolkenlosem Himmel senkrecht zur Wasseroberfläche gemessen. Im Mittel wurden am TQ I $15 \%$, am TQ II $17 \%$, am TQ III $7 \%$ und am TQ IV $13 \%$ der Helligkeit am Wasserspiegel ermittelt. Der vergleichsweise niedrige Wert am TQ III ist durch die Lage an einer senkrechten, teilweise überhängenden Wand bedingt.

\section{METHODIK}

Zur quantitativen Erfassung von Besiedlungsstrukturen des sessilen Benthos stehen grundsätzlich die Quadrat- und Transektmethode zur Verfügung (Stoddart, 1969). Für eine realitätsgetreue Wiedergabe der Artenzusammensetzung und Kolonieanzahl in einer Zone ist erstere besonders geeignet (Mergner, 1979; Mergner \& Schuhmacher, 1981; Weinberg, 1981). Falls die graphische Wiedergabe der Quadrate naturgetreu erfolgt, kann sie zu jeder Zeit als Grundlage für statistische Erhebungen spezieller Art dienen. So ist auch die hier vorgelegte Kartierung der Testquadrate für weitere Auswertungen vorgesehen.

Bei der Quadratmethode wird gegenüber der Transektmethode gelegentlich der 
hohe Zeitaufwand für die Bestandsaufnahme unter Wasser als nachteilig empfunden (Bouchon, 1981). Daher wurde schon frühzeitig die photographische Erfassung der Artenzusammensetzung und Bedeckungsrate zu Hilfe genommen (Drew: in Barnes et al., 1971; Bohnsack, 1979; Mergner \& Schuhmacher, 1981; Weinberg, 1981) und als schnelle und zuverlässige Methode anerkannt (Littler, 1971). Bei komplizierter Oberflächenstruktur und großer Artenvielfalt ist allerdings die Auswertung allein nach Photographien nicht hinreichend. Von uns wurde daher die photographische Erfassung mit anschließender Kartierung und Kontrolle in situ kombiniert.

Die Quadratmethode projiziert dreidimensionale Wuchsgebilde in eine Ebene; sie gibt also (ebenso wie die Transektmethode) die tatsächliche Oberfläche nicht wieder. Falls diese gefragt ist, muß mit Faktoren - je nach Annäherung der Wuchsform an einfache dreidimensionale geometrische Körper - gerechnet werden (Dahl, 1973; Pichon, 1978a).

Unter den Formen der Wasserbewegung beeinflussen insbesondere die Brandung mit ihren Brechern, Restwellen und Rücklaufströmen, aber auch einseitig gerichtete Rifflängsströmungen die Struktur, Entwicklung und Ausrichtung der Korallengemeinschaften. Wenn also dieser Einfluß analysiert werden soll, müssen zweckmäßigerweise Testflächen teils gegen die gemittelte Hauptrichtung der Wasserbewegung, teils von ihr abgewandt ausgesucht und die jeweiligen Unterschiede der Besiedlungsstruktur verdeutlicht werden.

Ein instruktives Beispiel liefert der Vergleich von Korallengemeinschaften auf den vier Riffhängen eines Atollprofiles, vor allem wenn dieses möglichst genau der gemittelten Hauptrichtung aller wesentlichen Wasserversetzungen folgt. Im Falle des Sanganeb-Atolls ist das die Winkelhalbierende zwischen den Richtungen der monsunbedingten Meeresströmungen, der vorherrschenden Winde und der Tag-Nachtbrise. Sie verläuft von SSW nach NNO. In ihrer Verlängerung zur Küste schneidet sie den SWPfeiler des Wingate-Barriereriffes und trifft auf das Küstensaumriff am Leuchtturm südostwärts der Hafeneinfahrt von Port Sudan (Abb. 1). Dabei werden von der gedachten Linie wechselweise gegen die Hauptwasserbewegung gestellte und von ihr abgekehrte Riffhänge getroffen, auf denen die Testareale ausgewählt wurden. Von den insgesamt sechs Probeflächen wurden für die vorliegende Untersuchung jedoch nur die vier Sanganeb-Areale näher analysiert. Jedes Areal sollte ein charakteristisches Teilstück des betreffenden Riffhanges repräsentieren. Hierzu wurde zunächst ein gewisser Abschnitt des Riffhanges überprüft, dann aber das Areal selbst in ca. $10 \mathrm{~m}$ Tiefe eher zufallsmäßig aus diesem ausgewählt und abgesteckt.

Die Abgrenzung mit Hilfe je eines $5 \times 5 \mathrm{~m}$ Netzes aus Nylonleinen mit 25 "Maschen" à $1 \mathrm{~m}^{2}$ erfolgte wie bei Mergner \& Schuhmacher (1981) beschrieben. Jedes Einzelquadrat wurde zentrisch photographiert (Nikonos III, $28 \mathrm{~mm}$ Objektiv). Neu gegenüber der herkömmlichen photographischen Erfassung war die anschließende Verifizierung vor dem Objekt unter Wasser: Die Aufnahmen der Einzelquadrate wurden auf wasserfestes Papier $(18 \times 18 \mathrm{~cm})$ abgezogen. Ein solcher Papierabzug eines jeden Einzelquadrates wurde zwischen zwei Kunststoffplatten geklemmt (Abb. 6b), von denen die obere als transparente Unterwasserschreibtafel diente. Die Umrisse der Korallenkolonien wurden auf der transparenten Schreibtafel gemäß dem darunterliegenden Photo nachgefahren - so konnten in der Photographie undeutliche Stellen sofort in situ geklärt werden. Gleichzeitig erfolgte die Identifizierung der einzelnen sessilen Organismen. 
H. Mergner \& H. Schuhmacher

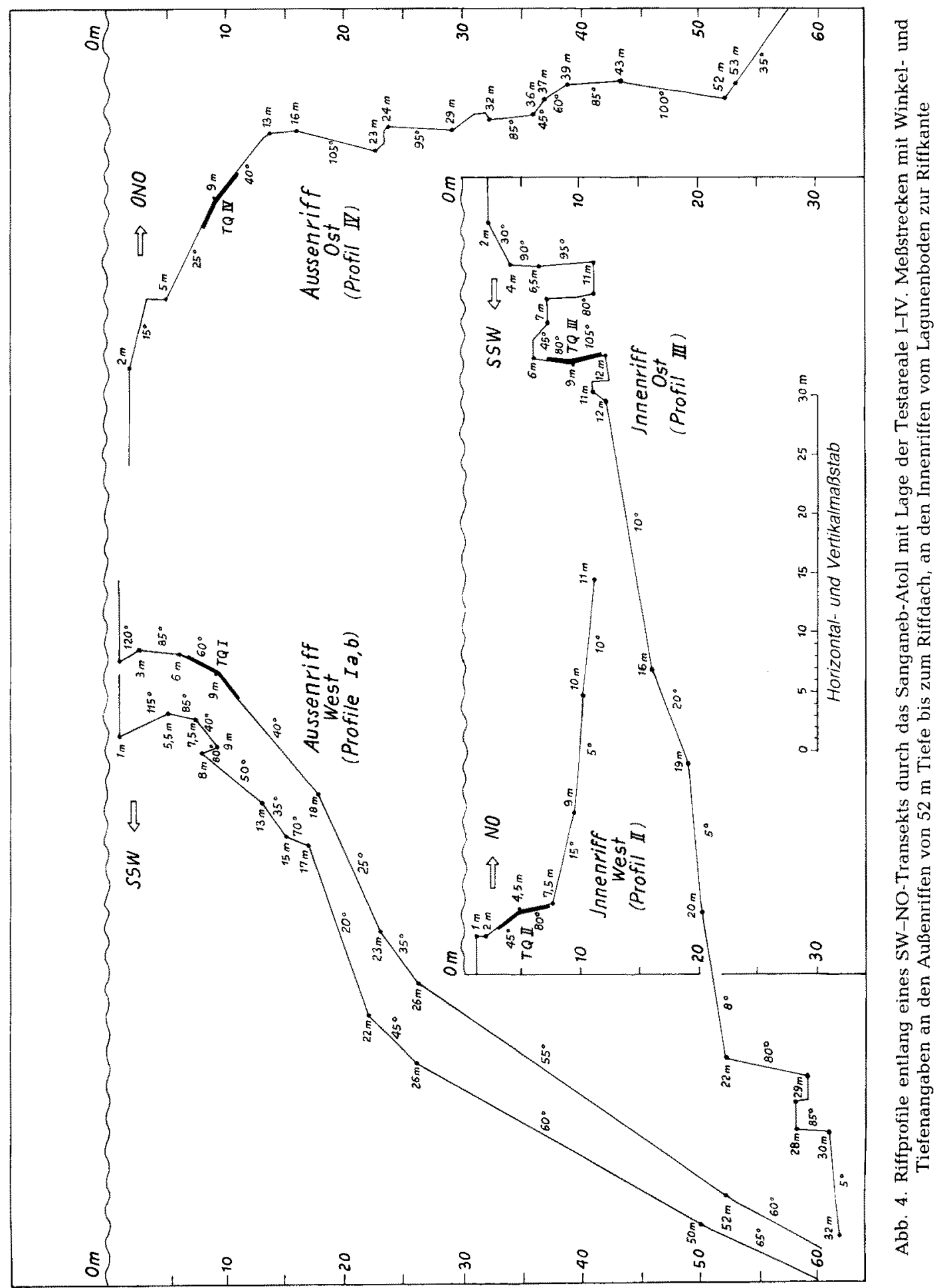


Analyse von Korallengemeinschaften. I.

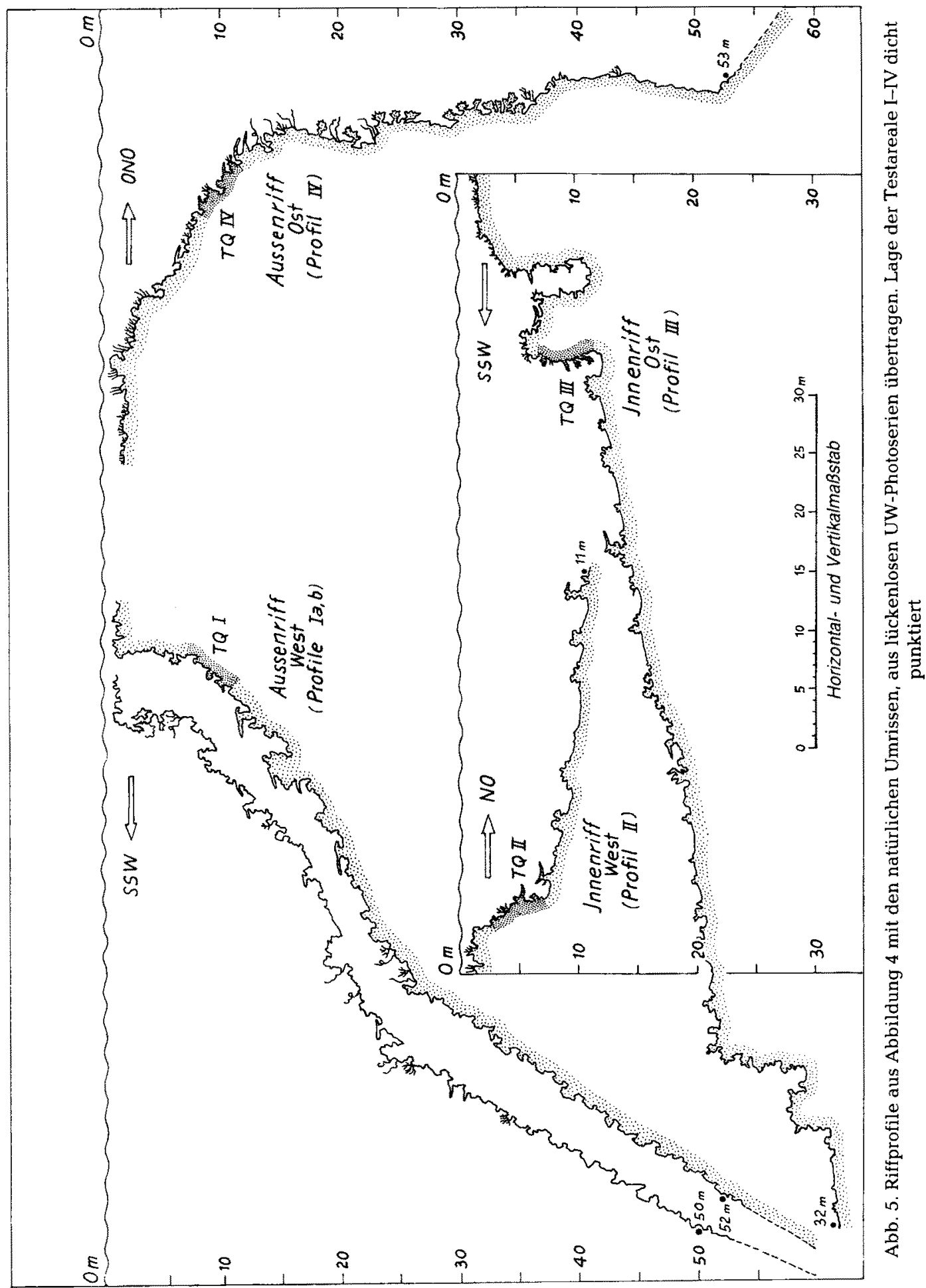


Falls das nicht möglich war, wurden numerierte Proben für eine spätere taxonomische Bearbeitung genommen.

Die auf diese Weise kartierten 25 Einzelquadrate wurden anschließend zeichnerisch zusammengefaßt. Der relative Anteil der jeweiligen sessilen Arten an der projizierten Grundfläche wurde ermittelt, indem in der Kartenzeichnung die Einzelkolonien
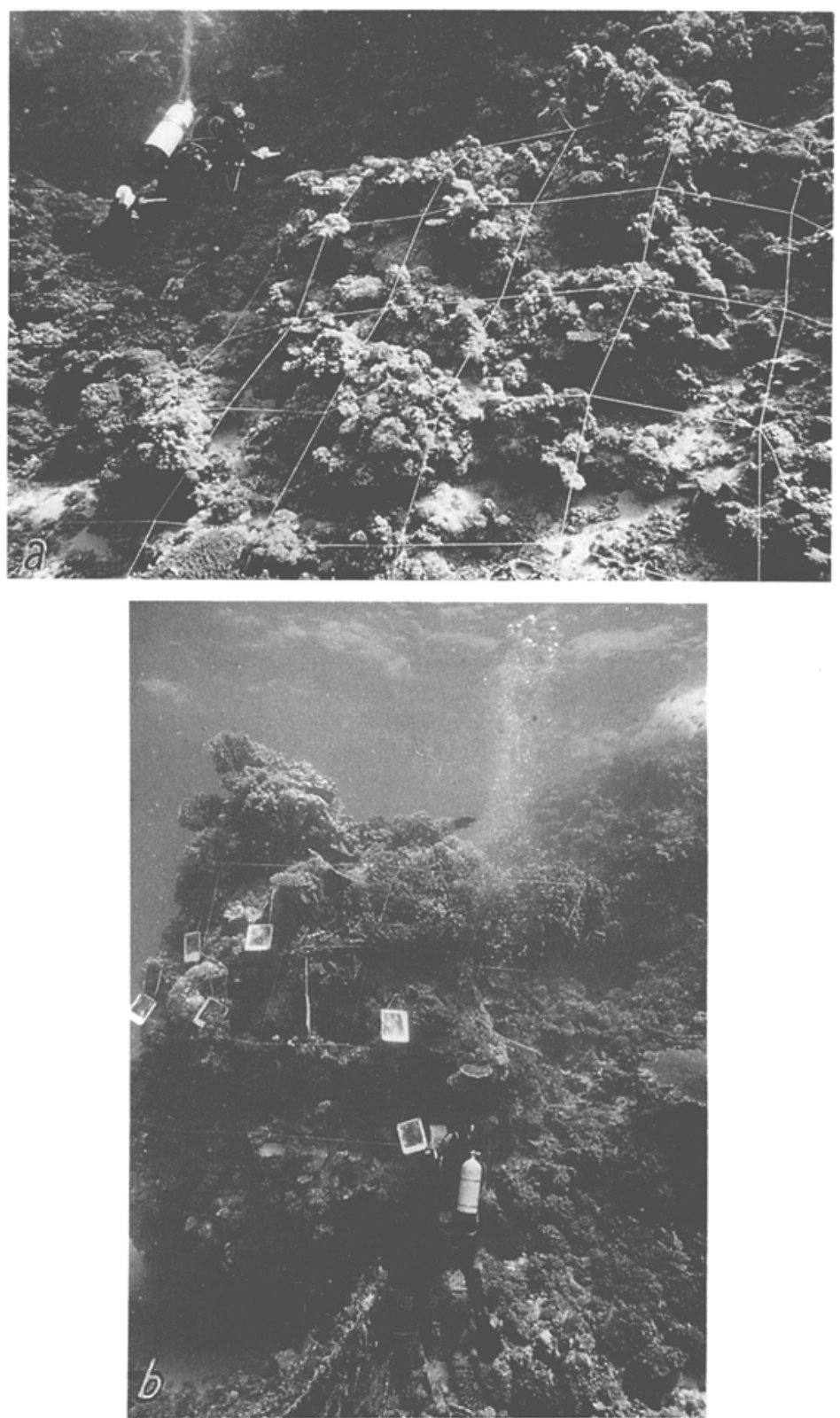

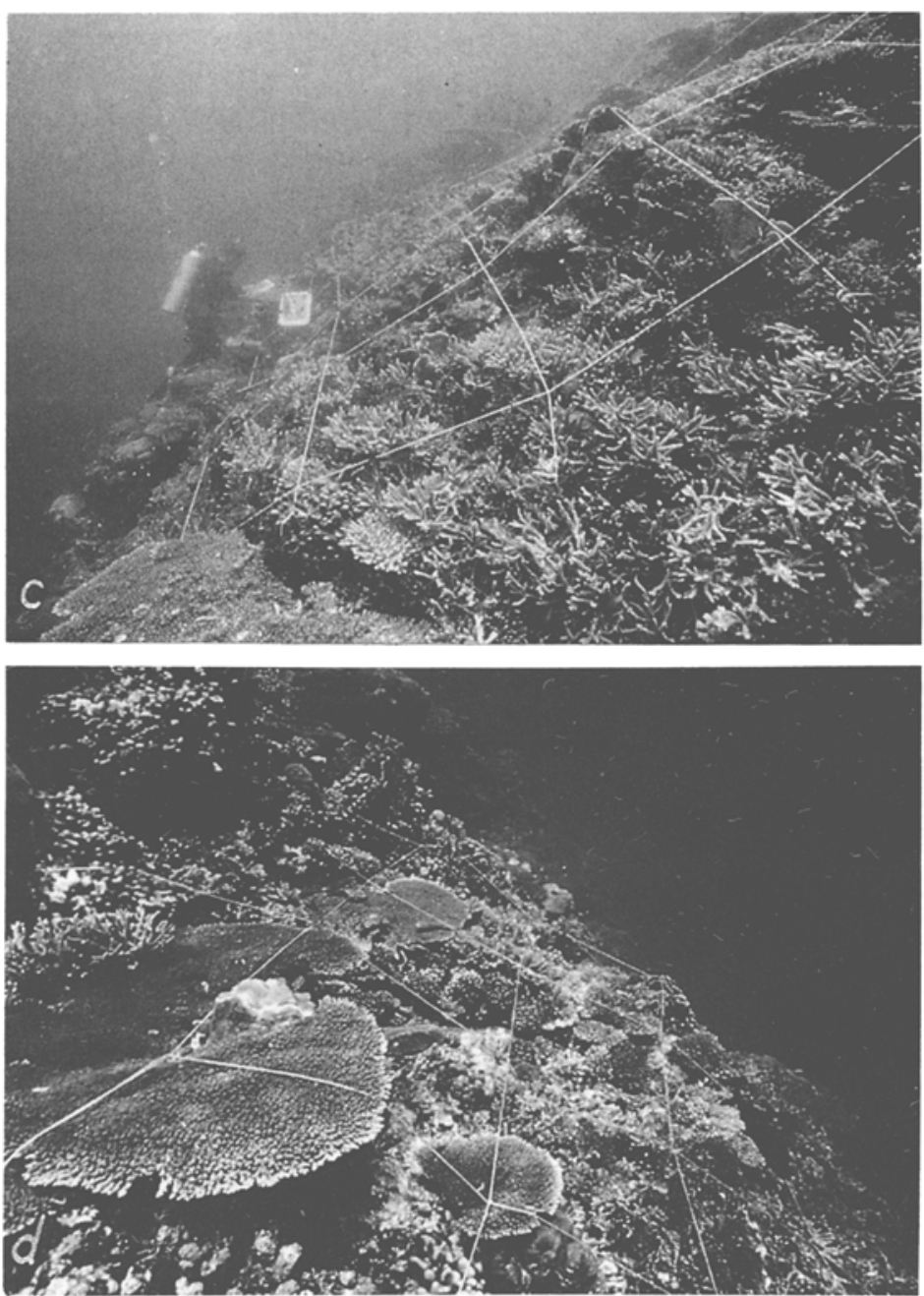

Abb. 6. UW-Photographien der Testareale I, III und IV. (a) TQ I am SW-Außenriff (Blick von SO). (b) TQ III am NO-Innenriff (Blick von S). (c) TQ IV am NO-Außenriff (Blick von N). (d) TQ IV am NO-Außenriff (Blick von S)

bzw. Einzelflächen entlang ihrer Umrisse ausgeschnitten und ihr Gewicht zu dem der Gesamtfläche in Beziehung gesetzt wurde. Die anschließende Auswertung betraf Artenzahl, Koloniezahl je Art und Koloniegröße (Projektion in die Ebene). Die Artendiversität wurde nach dem Index von Shannon und Wiener $\mathrm{H}^{\prime}=-\sum_{i=1}^{N} \mathrm{pi}^{\mathrm{i}} \cdot \ln$ pi und die Eveness nach Pielou (1966) als $I=\frac{H}{\ln N}$ berechnet. 


\section{ERGEBNISSE}

\section{Topographische und strukturelle Charakterisierung der Testquadrate}

Das in nordnordöstlicher Richtung (genau: mit $23^{\circ}$ über Nord) durch das SanganebAtoll gelegte Profil (Abb. 1 und 2) beginnt an der SW-Ecke und zieht zur Mitte der Ostseite. In seinem Verlauf finden sich auf den Außen- und Innenhängen des Atolls nacheinander die vier Testquadrate, deren Aufbau und Struktur im folgenden charakterisiert werden sollen:

T e st quadrat (TQ) I ist nahe der Südwestecke des Atolls in 6,5 bis $11 \mathrm{~m}$ Tiefe (Mittelwert: 8,75 m) auf dem Außenhang gelegen und gegen SSW ausgerichtet (Abb. 3b). Seine Hangneigung beträgt zwischen 6,5 und $9 \mathrm{~m}$ Tiefe etwa $60^{\circ}$, zwischen 9 und 11 m $40^{\circ}$ (Abb. 4 und 5). Darunter verflacht der Riffhang, von kleineren Steilstufen unterbrochen, noch weiter auf 25 bis $20^{\circ}$. Erst bei 23 bis $26 \mathrm{~m}$ Tiefe fällt er mit einem deutlichen Knick und 55 bis $65^{\circ}$ Hangneigung steil in eine auch bei $52 \mathrm{~m}$ nicht mehr einzusehende Tiefe. Die oberen Steilhänge des Riffprofils bestehen vorwiegend aus stark erodiertem Korallenfels, die tiefer gelegenen aus Blockhalden, deren mächtige Korallenfelstrümmer mit zunehmender Tiefe einen immer spärlicheren Lebendwuchs aufweisen. Zwischen die Blöcke sind kleine Sandinseln und Sandrinnen eingesprengt, und nur in den flacheren mittleren Tiefen des Hanges (18 bis $26 \mathrm{~m}$ ) finden sich auch größere Flächen mit Grob- und Mittelsand, in die einzelne große, meist üppig besiedelte Korallenfelshorste und Trümmer eingelagert sind.

Die Ubersichtaufnahme Abbildung 6a zeigt die Lage des Testquadrates I im oberen Bereich dieses Riffprofiles, während Abbildung 7 einen Eindruck von seinem strukturellen Aufbau gibt: Im oberen, steileren Teil des Areals finden sich zahlreiche lichtsuchende und daher in den freien Raum vorkragende Korallenkolonien als lebender Überzug stark gegliederter Korallenfelsbauten, mit denen sie infolge dieser Wuchsweise mehrfach übereinander breite, horizontale Simse bilden. In den tief beschatteten Höhlungen darunter zeigen Kalkrotalgen und Schwämme oft ausgedehnte Bewuchsflächen. Im unteren flacheren Teil des Untersuchungsareals treten dagegen die dicht besiedelten Querbänder etwas zurück zugunsten nunmehr eingesprengter Korallenschutthalden und Sandrutschen. In ihrem Bereich können nur wenige hochragende Korallenkolonien überleben, unter ihnen eine fast einen Quadratmeter große Acropora pharaonis-Kolonie.

T e s t a r e a 1 I, nach NO ausgerichtet, ist in 3,5 bis 7,5 m Tiefe (Mittelwert: $5,5 \mathrm{~m}$ ) auf dem westlichen Innenhang gelegen, dicht südlich der bis $14 \mathrm{~m}$ tiefen Westpassage des Atolls (Abb. 3c). Die Hangneigung in TQ II beträgt im oberen Teil $45^{\circ}$, ab $5 \mathrm{~m}$ Tiefe $80^{\circ}$. Vom Fußende des Areals fällt der Lagunenboden zunächst mit $15^{\circ}$ Neigung und ab $9 \mathrm{~m}$ Tiefe nur noch mit $5^{\circ}$ sehr allmählich gegen die Lagunenmitte hin ab (Abb. 4 und 5). Er besteht unmittelbar unter dem Testareal aus erodiertem, von Sandinseln und Schutt bedecktem Korallenfels, geht aber bald darauf in eine zusammenhängende, tief von Feinsand und Mulm überlagerte, wellige Ebene über. Aus ihr ragen nurmehr vereinzelte Korallenfelshorste bis $2 \mathrm{~m}$ Höhe mit meist üppigem Lebendbewuchs empor. Uber die Nord-Süd-Erstreckung der Atollagune hinweg und durch die offene Westpassage können die meist nördlichen Winde im Untersuchungsbereich eine kräftige Südostströmung und Brandung verursachen. Infolgedessen ist das feinsandige Sediment des hier 


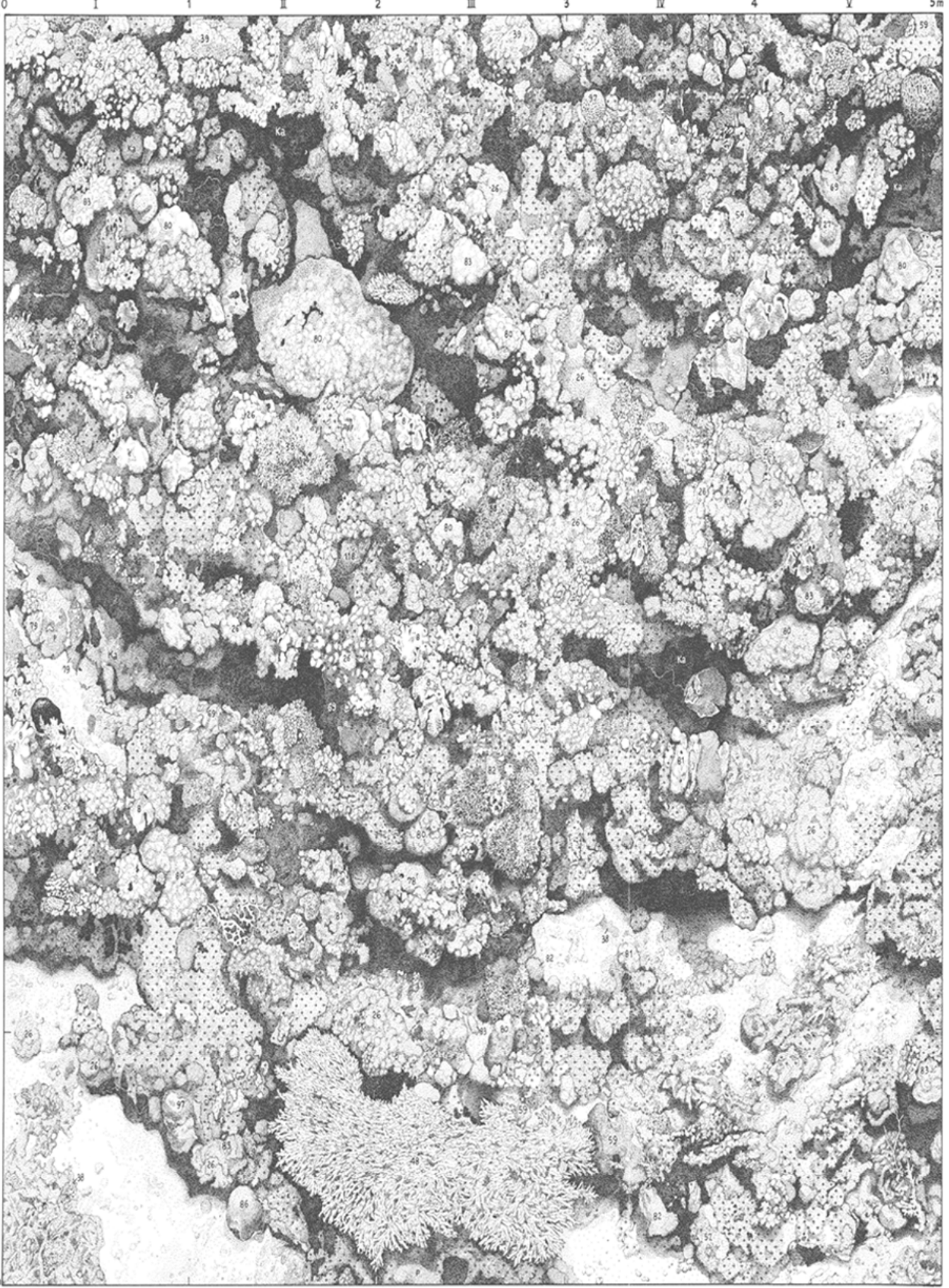

Abb. 7. Struktureller Aufbau der Korallengemeinschaft in Testareal I am Sanganeb-Atoll.

Maßstab 1:25. Die angegebenen Artnummern beziehen sich auf Tab. 2 


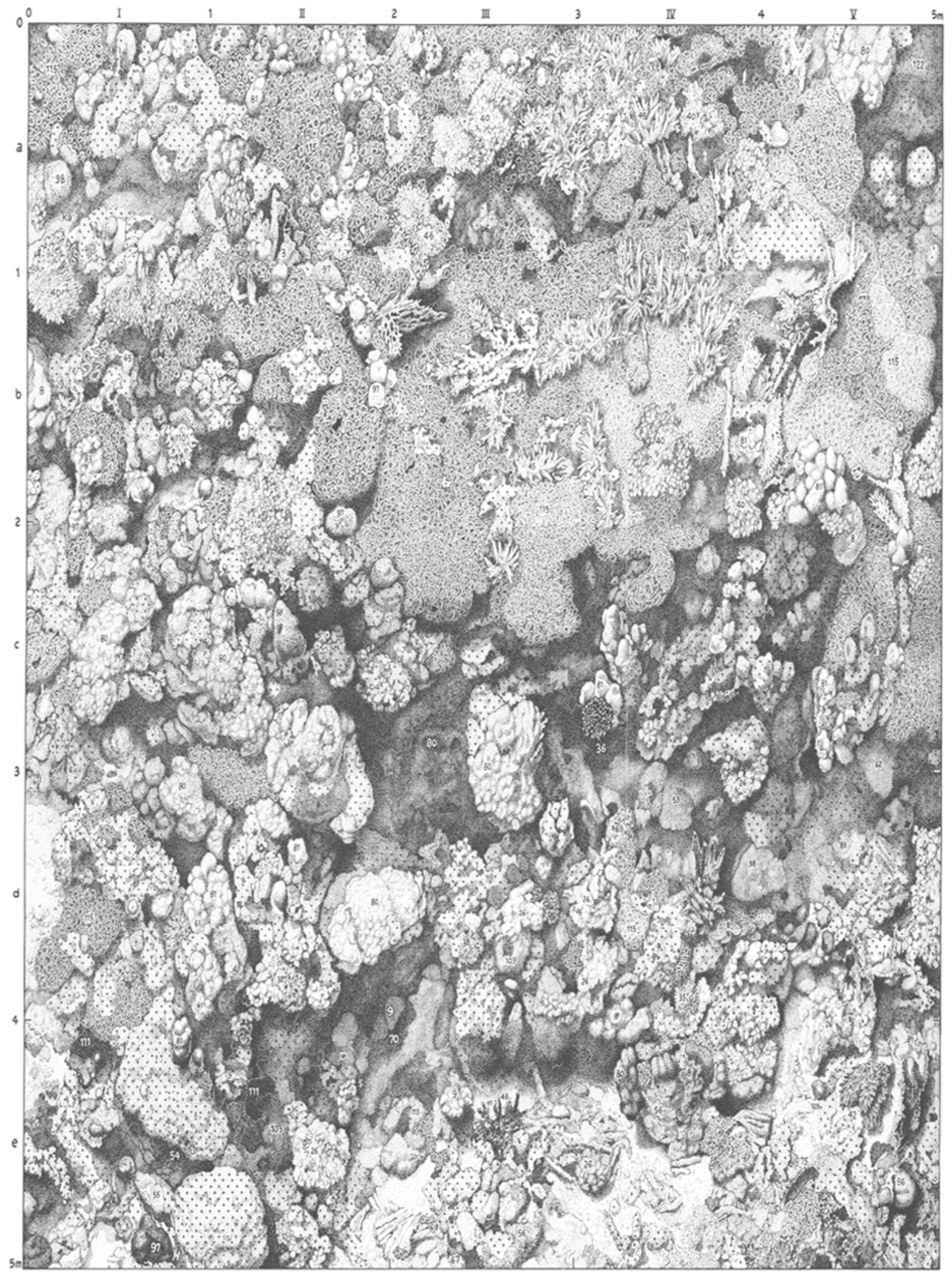

Abb. 8. Struktureller Aufbau der Korallengemeinschaft in Testareal II am Sanganeb-Atoll.

Maßstab 1:25. Die angegebenen Artmummern beziehen sich auf Tab. 2 


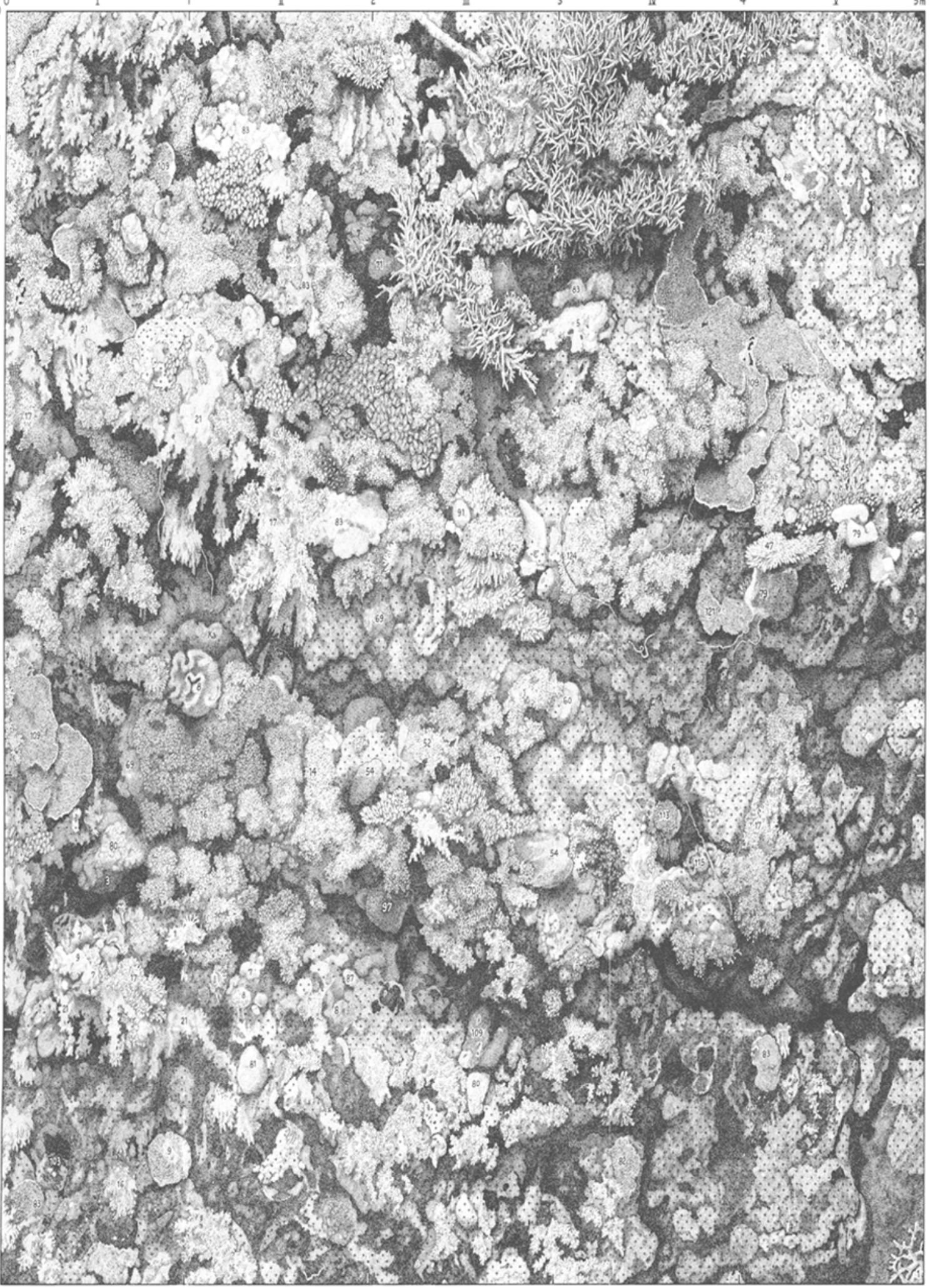

Abb. 9. Struktureller Aufbau der Korallengemeinschaft in Testareal III am Sanganeb-Atoll.

Maßstab 1:25. Die angegebenen Artnummern beziehen sich auf Tab. 2 


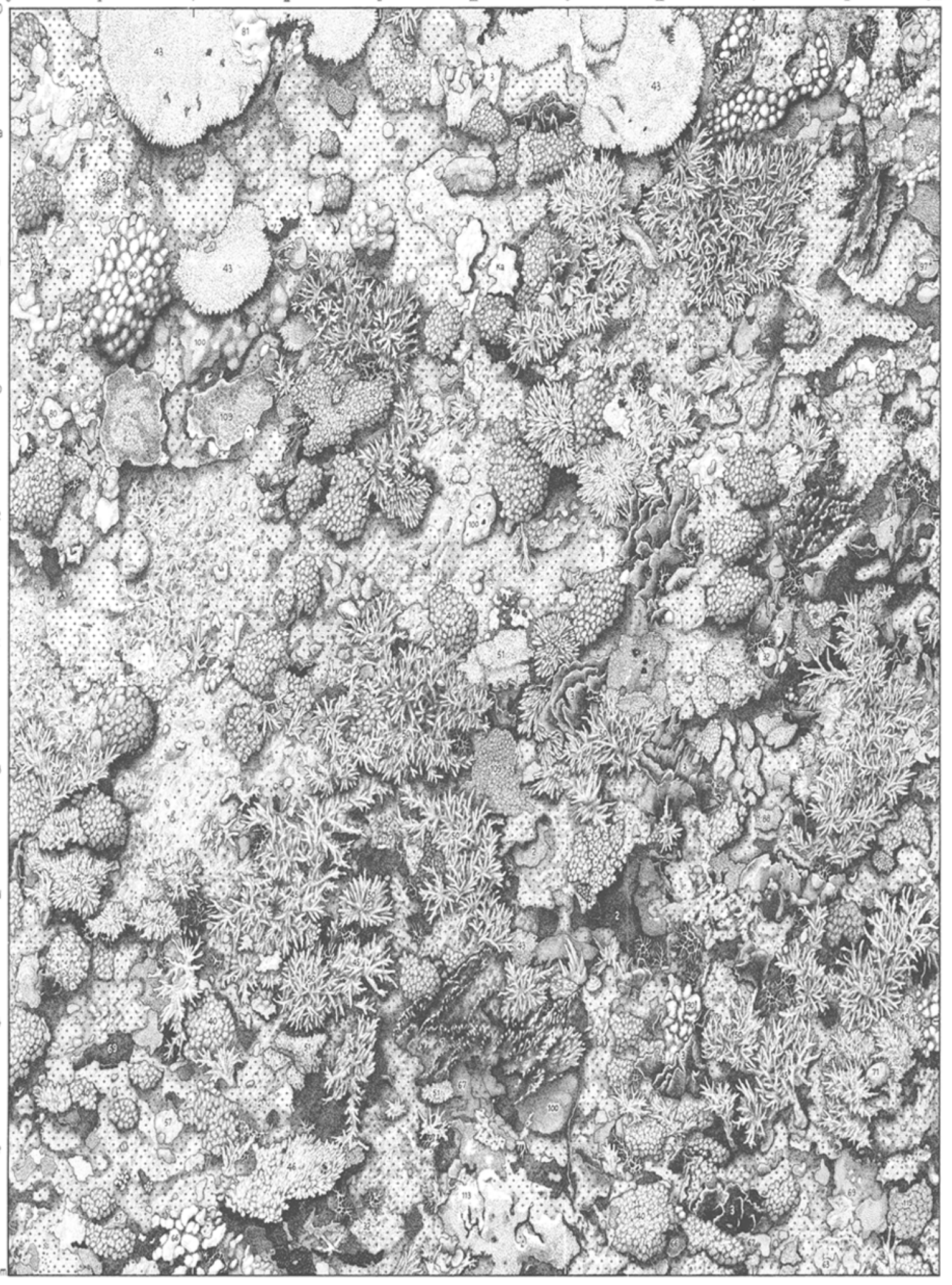

Abb. 10. Struktureller Aufbau der Korallengemeinschaft in Testareal IV am Sanganeb-Atoll.

Maßstab 1:25. Die angegebenen Artnummern beziehen sich auf Tab. 2 
bis $10 \mathrm{~m}$ tiefen Lagunenbodens fast immer etwas aufgewühlt und die mittlere Sichtweite unter Wasser beträgt nur etwa $9 \mathrm{~m}$.

Noch deutlicher als bei TQ I zeigt die zeichnerische Strukturanalyse von Testareal II (Abb. 8) zwei unterschiedlich geneigte Bereiche, deren oberer Teil mit $45^{\circ}$ Hangneigung wesentlich flacher ist als der untere mit $80^{\circ}$. Dieser Unterschied manifestiert sich auch in der Korallenbesiedlung: Die flachere, besser beleuchtete und der Wasserbewegung stärker ausgesetzte, obere Hälfte wird durch ausgedehnte, oft lückenlos zusammengewachsene Kolonien von Lobophyllia corymbosa gekennzeichnet (Abb. 11c). Dazwischen finden sich im wesentlichen nur zahlreiche, gegen die Längsströmung gestellte Fächer der Millepora dichotoma und Bestände von Acropora hemprichi. Unterhalb der Lobophyllia-Flächen öffnen sich im steilen Abschnitt des Areals umfangreiche und tiefe Höhlungen mit einzelnen weit in den freien Raum vordringenden Korallenbauten (Abb. 11d), die vor allem von Porites lutea und $P$. solida-Blöcken gebildet und oft von Montipora effusa und $M$. meandrina überkrustet sind. Am Fuß des Steilhanges breiten sich wiederum Sandflächen mit Korallenschutt aus, vor allem mit den charakteristischen Bruchstücken der Lobophyllia-Kolonien. Bei keinem anderen Testareal lassen sich zwei so deutlich gegeneinander abgegrenzte Zonen unterscheiden (Abb. 8).

An den beiden Ausgängen einer schmalen Passage auf der Ostseite des Atolls, die kleinen Booten das Queren des Riffdaches erlaubt, wurden die restlichen Testquadrate festgelegt, TQ IV auf der Außenseite und TQ III auf der Lagunenseite (Abb. 1, 2 und 3d).

Testareal III, nach SSW ausgerichtet, ist in 7 bis 11,5 m Tiefe (Mittelwert: $9,25 \mathrm{~m}$ ) auf dem lagunenseitigen Riffhang der Ostseite, etwas nördlich ihrer Mitte, gelegen (Abb. 3d und 6b). In dieser Region bleiben auf mehr als $150 \mathrm{~m}$ Länge Riffdach und Riffkante 1-2 m unter der Wasseroberfläche und branden deshalb nur bei hohem Seegang. Infolge der Vertiefung wird bei den vorherrschenden Nordwinden größeren Windwellen der Durchzug vom offenen Meer der Außenseite in die Atollagune ermöglicht.

Zum Lagunenboden fällt die östliche Riffbarriere im weiteren Untersuchungsbereich meist senkrecht auf 10 und mehr Meter Tiefe ab (Abb. 4 und 5), wobei die Korallenfelswände oft stark zerklüftet oder sogar, wie im TQ III, pfeilerartig abgetrennt sein können. Vor allem im Nordostteil der Atollagune finden sich schluchtartig tiefeingeschnittene und weitverzweigte Canyonsysteme. Am Testareal III weist der bearbeitete Innenhang zwischen 7 und $9 \mathrm{~m}$ Tiefe $80^{\circ}$ Hangneigung auf und darunter bis zum Fußende in $11,5 \mathrm{~m}$ Tiefe $105^{\circ}$. Von hier ab neigt sich der Lagunenboden mit 10 bis $5^{\circ}$ nur noch sehr geringfügig zur Lagunenmitte hin. Er besteht teils aus Grob- und Mittelsanden mit einzelnen Korallenfelshorsten, welche mit ihrem Lebendbewuchs die weiten Sandflächen mit bis zu $0,8 \mathrm{~m}$ Höhe überragen, teils aus Korallenfelsflächen mit eingelagerten kleinen Sandinseln. Bei etwa $60 \mathrm{~m}$ Entfernung von TQ III zur Lagunenmitte und 22 bis $23 \mathrm{~m}$ Tiefe fällt der Korallenfelsboden abrupt über eine arenaförmig-halbrund angeordnete, mehrfach gegliederte und tief zerklüftete Steilstufe mit 80 bis $85^{\circ}$ Hangneigung auf $31 \mathrm{~m}$ Tiefe ab. Erst dann setzt sich der Lagunenboden wieder mit geringem Gefälle (bis $5^{\circ}$ ) als feinsandige Ebene fort.

Die zeichnerische Strukturanalyse des Testareals III (Abb. 9) läßt seinen gegenüber den übrigen TQs deutlich abweichenden strukturellen Aufbau sofort erkennen: Zwar bestimmen im oberen Drittel der Untersuchungsfläche bis etwa 8,5 m Tiefe, vor allem im östlichen (rechten) Teil, der dem Einfluß der über das Riffdach hinweglaufenden 
Windwellen (und des von ihnen erzeugten vertikalen Wasseraustausches) am stärksten ausgesetzt ist, zumeist Steinkorallen diesen Aufbau: Größere Bestände von Acropora hemprichi, umfangreiche Krusten von Echinopora gemmacea, Symphyllia erythraea, Fächerkolonien von Millepora dichotoma u. a. Aber im größten Teil des Testareals herrschen Weichkorallen mit $2 / 3$ des gesamten Cnidaria-Bestandes vor, darunter allein Sinularia polydactyla mit einem Drittel dieses Bestandes und Dendronephthya hemprichi mit einem Zehntel. Dazu kommen noch weitere Sinularia-Arten (mit fast $15 \%$ ) und Parerythropodium fulvum (mit $4 \%$ des Bestandes). Vor allem am senkrechten Riffhang links oben und in den ganzen unteren Zweidritteln der hier überhängenden Riffwand dominieren fast nur Weichkorallen. Unaufgepumpt hängen vor allem die großlappigen Dendronephthya-Stöcke schlaff herab und überdecken z. T. andere sedentäre Besiedler der Riffwand. Zusammen mit den ebenfalls bei Tag stark kontrahierten SinulariaKolonien (Abb. 11e) "verschleiern" sie den Hartstrukturcharakter der Riffwand, so daß sich dieses Testareal nur schwer mit den Strukturbesonderheiten der drei übrigen vergleichen läßt. Trotzdem treten wie bei TQ I auch hier mehrere, von den wenigen Steinkorallen aufgebaute Querrippen hervor, wenn auch nicht so ausgeprägt simsartig wie im ersten Beispiel und vorwiegend aus totem Korallenfels bestehend. Sand- und Schutthalden sind an dieser größtenteils überhängenden Riffwand natürlich nicht zu finden, sondern nur an ihrer Basis in $12 \mathrm{~m}$ Tiefe. Die Ubersichtsaufnahme Abbildung $6 \mathrm{~b}$ gibt nochmals einen Eindruck von der besonderen Lage und Struktur des Testareals III.

Testareal IV ist zwischen 8 und $11,5 \mathrm{~m}$ Tiefe (Mittelwert: 9,75 m) auf dem Außenhang der Ostseite gelegen, ungefähr am Ostende der vom TQ III ostnordostwärts über das Riffdach führenden Bootspassage und etwa $120 \mathrm{~m}$ von diesem entfernt (Abb. 3d). Im engeren Untersuchungsbereich zeigt der nach ONO ausgerichtete Riffhang oben eine Neigung von nur $25^{\circ}$, unten jedoch von $40^{\circ}$ (Abb. $6 \mathrm{c}$ ). Ab $13 \mathrm{~m}$ Tiefe fällt das Riff bei nur 2,5 m horizontaler Abweichung auf $52 \mathrm{~m}$ Tiefe ab. Von dort ist das untere Ende des $35^{\circ}$ geneigten Schutthanges aus Grobsand, Korallenbruch und Muschelschill trotz einer UW-Sichtweite von mehr als $35 \mathrm{~m}$ nicht mehr zu erkennen. Der riesige Steilabfall (Abb. 4) selbst besteht aus drei großen Überhängen, nämlich zwischen 16 und $23 \mathrm{~m}$ Tiefe mit $105^{\circ}$ Neigung, zwischen 24 und $29 \mathrm{~m}$ mit $95^{\circ}$ und zwischen 43 und $52 \mathrm{~m}$ mit $100^{\circ}$. Die Zwischenstrecken weisen eine Hangneigung von jeweils $85^{\circ}$ auf, nur von schmalen Korallenfelsbändern und schrägen Simsen unterbrochen. Bei 23 bis $24 \mathrm{~m}$ Tiefe zeigt der eindrucksvolle Riffabsturz eine $2 \mathrm{~m}$ breite Stufe, möglicherweise eine Brandungsterrasse bei eiszeitlichem Meerestiefstand.

Das oben beschriebene Riffprofil zum Testareal IV (Abb. 5) zeigt deutliche biophysiographische Zonenbildungen (Begriffserläuterung bei Mergner \& Schuhmacher, 1974): Die Sedimenthalde unterhalb $52 \mathrm{~m}$ Tiefe läßt kaum Lebendbewuchs erkennen. Auf der Korallenfelswand darüber bis etwa $39 \mathrm{~m}$ findet sich nur eine lückenhafte Besiedlung durch krustenförmig wachsende Kalkrotalgen, lappig-flächige Montiporaund Echinopora-Arten, Xeniiden, Poriferen und feinfiederige Antipatharien. Weiter oben bis annähernd $24 \mathrm{~m}$ bilden Gorgonarien (wahrscheinlich Acabaria spp.) mit ihren gegen die Rifflängsströmung gestellten Fächern dichte Wälder. Zu ihnen kommen wiederum Antipatharien, während die Riffwand selbst mit ausgedehnten Krusten von Rotalgen, Schwämmen und Steinkorallen bedeckt ist. Darüber treten bis $16 \mathrm{~m}$ vermehrt die auffallenden Cirripathes-Peitschen auf, aber auch schon oft kandelaberartige Kolonien von Acropora, Pocillopora, Porites, mehrere Weichkorallenarten wie Sinularia und 
Xeniiden und erste Millepora-Fächer. Zwischen 16 und $7 \mathrm{~m}$ Tiefe überwiegen umfangreiche Tische von Acropora corymbosa und A. hyacinthus sowie ausgedehnte Bestände von $A$. hemprichi und $A$. superba. Letztere wurde mit gewisser Zurückhaltung so identifiziert; denn seit Klunzinger (1879) wurde die Art nicht mehr nachgewiesen (vgl. Scheer \& Pillai, 1983). Außerdem charakterisieren auch zahlreiche Pocillopora verrucosa-Kolonien und Millepora-Stöcke diesen Tiefenbereich. Den obersten Riffhang und die Riffkante beherrschen Porites lutea, Pocillopora verrucosa, geweihförmig verzweigte Acropora-Arten wie $A$. hemprichi und $A$. superba und vor allem mehrfach gestaffelte Barrieren gegen die Brandung gestellter Millepora dichotoma-Fächer. Derart zusammengesetzte Korallengemeinschaften begleiten auch die von kräftigen Strömungen durchzogene und von oft hohen Windwellen überlaufene, 1 bis $3 \mathrm{~m}$ tiefe Bootspassage über das Riffdach. Sie gehen fast lückenlos über in die dicht ineinander verfilzten Acropora-Gebüsche des obersten Riffinnenhanges oberhalb von Testareal III und können geradezu als Indikator gelten für Bereiche ausgiebiger, aber nur selten zerstörender Wasserbewegung.

Einen ersten Eindruck von dieser charakteristischen Zweigkorallenlandschaft und der Lage des Testareals IV in ihr gibt die Übersichtsaufnahme Abb. 6c. Sie zeigt vor allem die dichte Erfüllung des Siedlungsraumes mit verzweigten Acropora-Arten auf dem hell beleuchteten oberen Außenhang des Ostriffs, unmittelbar über seinem Absturz auf $52 \mathrm{~m}$ Tiefe. Die zeichnerische Strukturanalyse (Abb. 10) des TQ IV macht offenbar, daß $75 \%$ aller hier siedelnden Steinkorallen zu den Zweigkorallen gehören, insbesondere zu tisch-, kandelaber- und geweihförmigen Acropora-Arten (Abb. 11f), blumenkohlförmig gedrungenen Pocillopora-Stöcken (Abb. 11g) und in Reihen stehenden bzw. ineinander geschachtelten Millepora-Kolinien (Abb. 11h). Erst bei genauerem Studium lassen sich Anzeichen einer wenigstens teilweise gesetzmäßigen Anordnung des Bewuchses erkennen: Ein leichter Knick in der Hangneigung zwischen einem oberen flacheren Teil $\left(25^{\circ}\right)$ und einem unteren steileren Teil $\left(40^{\circ}\right)$ zieht sich schräg durch das Areal von rechts oben nach links unten. Er trennt zwei unterschiedlich helle Bereiche voneinander und wird zusätzlich markiert durch Konzentrationen parallel stehender Millepora-Fächer, deren Breitseite nach rechts unten gegen die Hauptwasserbewegungsrichtung aus NNO gewendet ist. Sie bestätigen damit die Ausrichtung der Profillinie, längs der die Testquadrate festgelegt wurden, durch das Atoll von SSW nach NNO. Von allen vier Testquadraten finden sich in diesem die geringsten Flächenanteile an unbelebtem Substrat, also totem Korallenfels und Sediment. Lediglich im oberen Teil gibt es einige zusammenhängende Korallenfelspartien mit kleineren KorallenschuttAnsammlungen.

Bei allen Riffprofilen des Sanganeb-Atolls wurden mehr oder weniger deutlich ausgeprägte Knickstellen oder Stufen in ungefähr $23 \mathrm{~m}$ Tiefe festgestellt. Geringe Tiefendifferenzen von höchstens $2 \mathrm{~m}$ sind wahrscheinlich nur in der unterschiedlichen Genauigkeit des verwendeten Tiefenmessers begründet. Möglicherweise sind alle diese auffälligen Marken im Unterwasserprofil als Brandungshohlkehlen bzw. Brandungsterrassen eines eiszeitlichen Tiefstandes des Meeresspiegels zu deuten. Oder sie sind eine Folge spättertiärer epirogenetischer Bewegungen des Untergrundes bzw. der bei der Bildung des sog. "Erythräischen Grabens" entstandenen Horste. Auch am SW-Pfeiler des Wingate-Barriereriffs wurde ein deutlicher Profilknick in gleicher Tiefe gefunden. 

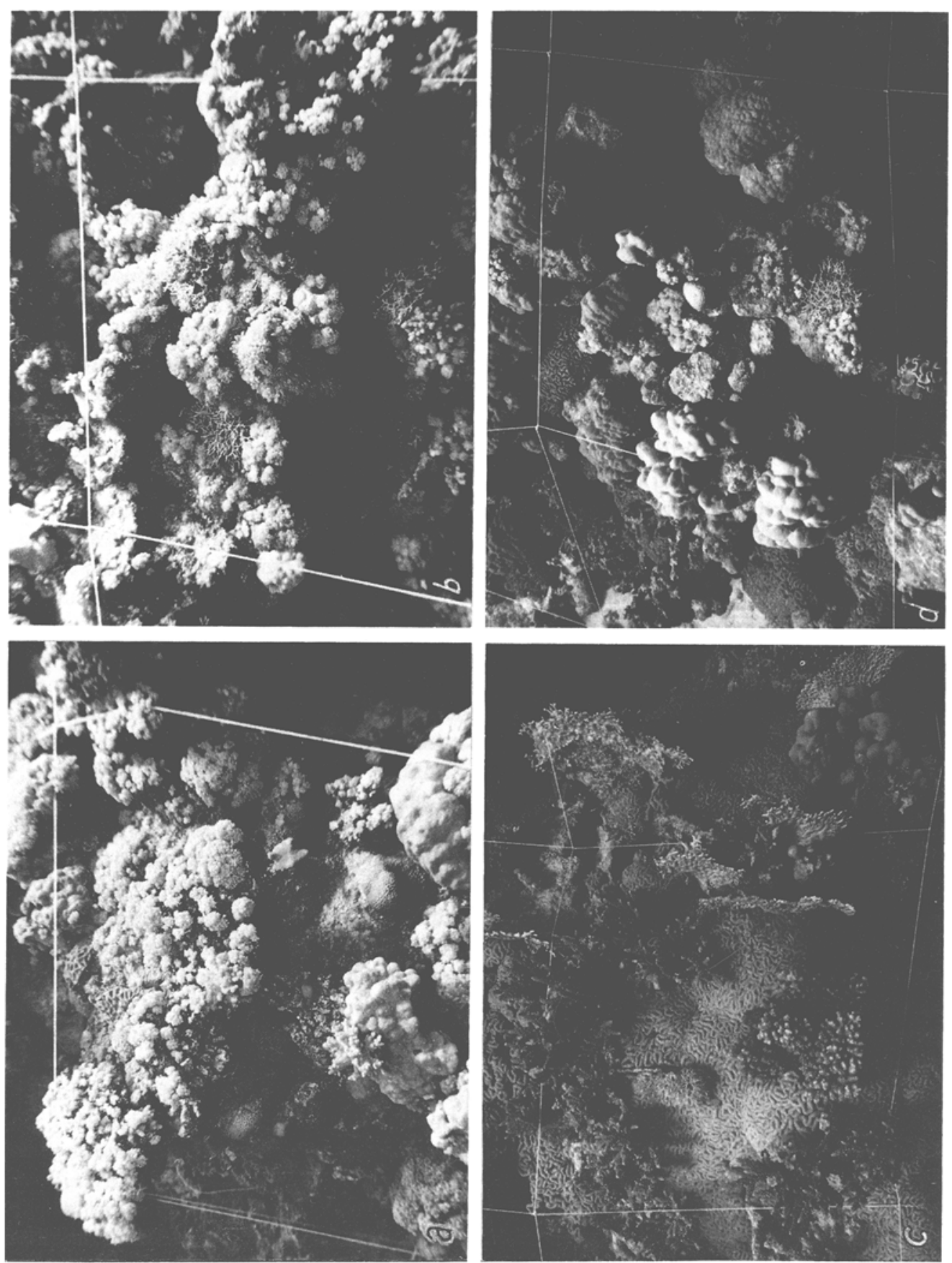

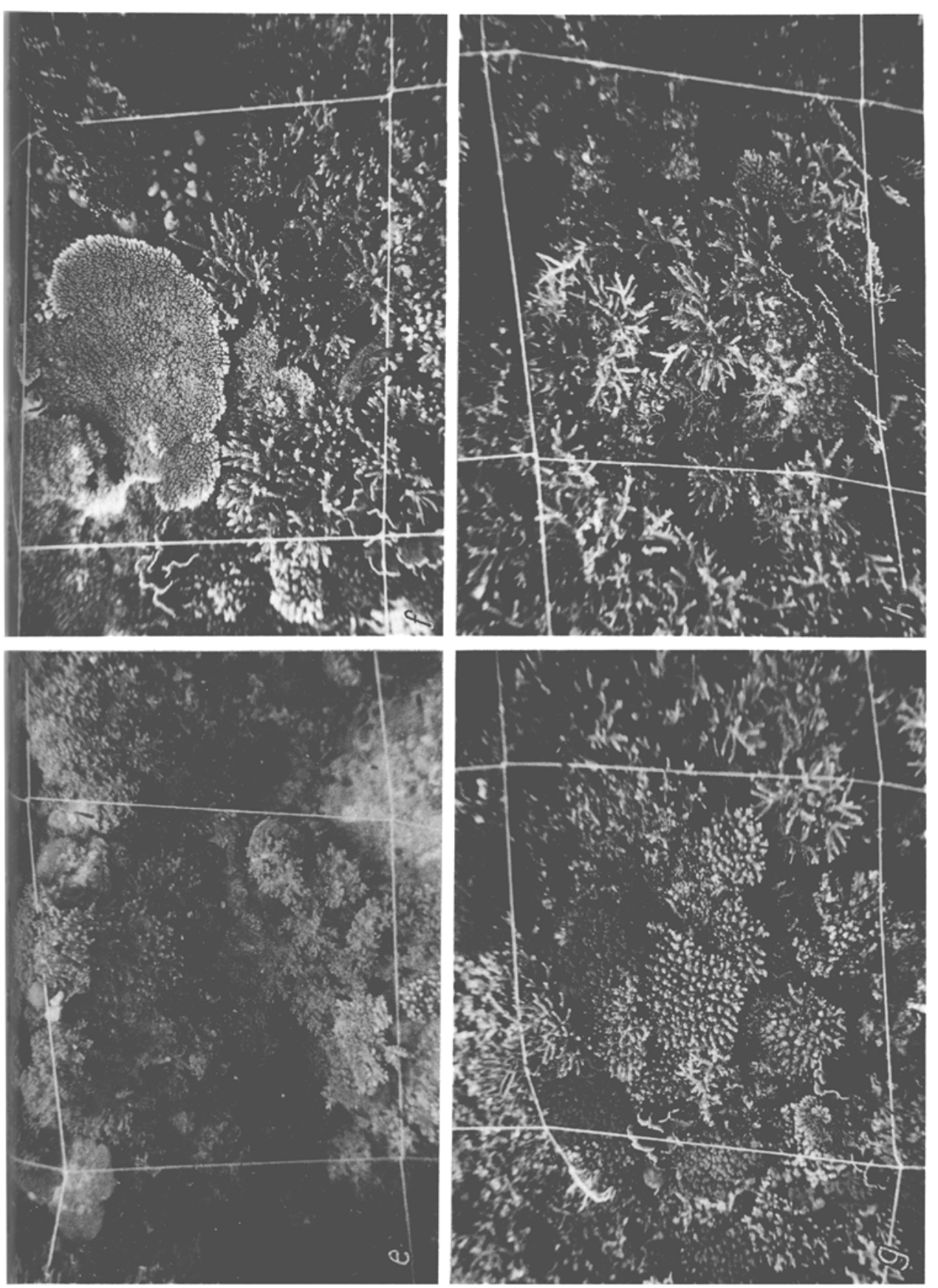

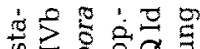
क人 웡

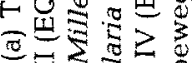
政

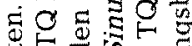
可证可

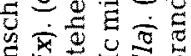

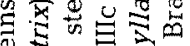
wo

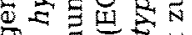

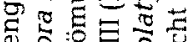
$=$ 녕 옹

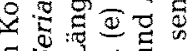
我

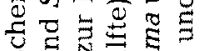

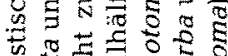
을

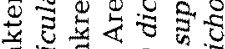
중

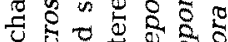

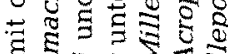
전

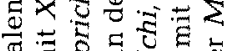
व 可 क⿺辶万 凷 园这 눙 o g वि ब $=$ मे 这的 음 대 5 .

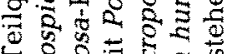
$\circ$. 政 5 용 \% 주의응

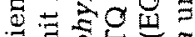
프응 c. 0 으응

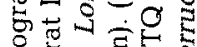

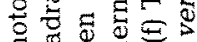

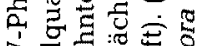

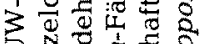
5 중 可思 - 西

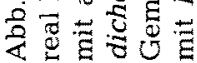




\section{Quantitative Analyse der Cnidaria-Besiedlung in den Testarealen}

Im vorangegangenen Abschnitt der topographischen und physiographischen Charakterisierung der vier Testareale entlang des Sanganeb-Profils wurde ein Zusammenhang zwischen Häufigkeit und Verlauf des Wasseraustausches und dem Vorherrschen bestimmter Stein- und Weichkorallen deutlich. Das nachfolgende Zahlenmaterial betrifft die sehr unterschiedliche Zusammensetzung und Diversität der Artenbestände, ihre Wuchsformen-Dominanz, ihre Verteilung und Bestandsbildung, das Hervortreten von Leitarten und biophysiographischen Zonen sowie Angaben zur Wuchsfläche einiger Gruppen der sedentären Begleitfauna und -flora wie Kalkrotalgen, Porifera und Bivalvia. Von über 3800 ausgewerteten Daten enthalten die Tabellen in beiden Teilarbeiten über 2600, anhand derer sich die quantitativen Analysen fast immer eindeutig absichern lassen.

\section{Artenbestand und Artenaufteilung}

Insgesamt wurden in den vier Testarealen $124^{*}$ Cnidaria-Arten aus 54 Gattungen festgestellt, nämlich 4 Arten (aus 2 Gattungen) der Hydrocorallia, 28 (15) der Octocorallia und 92 (37) der Hexacorallia (Tab. 2 und 3). Unter letzteren befinden sich allein 90 Arten der Scleractinia aus 35 Gattungen. Dazu kamen in unmittelbarer Umgebung der Testareale fast regelmäßig noch folgende Steinkorallen vor, die in keiner der tabellarischen Ubersichten enthalten sind: hermatypisch: Plerogyra sinuosa, Turbinaria mesenterina, ahermatypisch: Balanophyllia gemmifera, Tubastraea aurea, Tubastraea micranthus.

Unter den riffbildenden Scleractinia fanden sich, verglichen mit den Angaben von Head (1980), Kühlmann (1983) und Scheer \& Pillai (1983), für das mittlere Rote Meer 11 sichere und 2 fragliche Neufunde von Arten und der einer Varietät. Für das gesamte Rote Meer waren 3 Arten und die Varietät neu (vgl. Tab. 2).

Die 124 Arten der Cnidaria waren auf 2649 Kolonien bzw. Individuen verteilt. Von ihnen entfielen auf die einzelnen Testareale:

TQ I: 847 Kolonien mit $10,57 \mathrm{~m}^{2}$ Fläche $=42,4 \%$ von $25 \mathrm{~m}^{2}$;

TQ II: 652 Kolonien mit $14,15 \mathrm{~m}^{2}$ Fläche $=56,6 \%$ von $25 \mathrm{~m}^{2}$;

TQ III: 530 Kolonien mit $12,27 \mathrm{~m}^{2}$ Fläche $=49,1 \%$ von $25 \mathrm{~m}^{2}$;

TQ IV: 620 Kolonien mit $13,15 \mathrm{~m}^{2}$ Fläche $=52,8 \%$ von $25 \mathrm{~m}^{2}$.

Diese Kolonien verteilten sich in den Testarealen auf die wichtigsten CnidariaGruppen (Scleractinia, Alcyonaria, sonstige Cnidaria) wie in Tabelle 4 dargelegt.

\section{Begleitfauna und -flora}

Cnidaria bilden also mit einer mittleren Bestandsdichte von 662 Kolonien pro Testareal, einer mittleren Bodenbedeckung von $12,54 \mathrm{~m}^{2}$ und einem mittleren Gesamtflächenanteil von $50,2 \%$ das absolut überwiegende Element des Lebendbewuchses. Daneben spielt die sedentäre Begleitfauna und flora zumeist eine vergleichsweise geringe Rolle: So konnten nur gelegentlich auftretende kleine Kolonien von Ascidien,

* Unter den 124 Arten werden die Nominatform der Steinkorallenart Echinopora gemmacea und ihre Varietät $E$. gemmacea fruticulosa gesondert aufgeführt und gezählt, weil sie unterschiedlichen Kategorien von Wuchsformen, krustig-massigen bzw. verzweigten, angehören. Bei Angaben zur Gesamtartenzahl von Scleractinia und umfassenderen Taxa ist diese Doppelzählung zu berücksichtigen. 
Tab. 2. Besiedlung der Testareale des Sanganeb-Atolls durch Cnidaria

\begin{tabular}{|c|c|}
\hline HYDROZOA & ANTHOZOA, HEXACORALLIA \\
\hline Hydroida & Scleractinia \\
\hline Milleporidae & Thamnasteriidae \\
\hline 1 Millepora dichotoma Forskål & 33 Psammocora haimeana \\
\hline 2 Millepora exaesa Forskål & Milne Edwards \& Haime \\
\hline 3 Millepora platyphylla Ehrenberg & 34 Psammocora nierstraszi v. d. Horst* \\
\hline Stylasteridae & Astrocoeniidae \\
\hline 4 Distichopora violacea (Pallas) & 35 Stylocoeniella armata (Ehrenberg) ${ }^{*}$ \\
\hline ANTHOZOA, OCTOCORALLIA & $\begin{array}{l}\text { Pocilloporidae } \\
36 \text { Stylophora pistillata (Esner) }\end{array}$ \\
\hline Stolonifera & 37 Seriatopora caliendrum Ehrenberg * \\
\hline Tubiporidae & 38 Seriatopora hystrix Dana \\
\hline 5 Tubipora musica Linnaeus & 39 Pocillopora damicornis (Linnaeus) \\
\hline Alcyonaria & 40 Pocillopora verrucosa (Ellis \& Solander) \\
\hline Alcyoniidae & Acroporidae \\
\hline 6 Lobophytum pauciflorum (Ehrenberg) & 41 Astraeopora myriophthalma (Lamarck) \\
\hline 7 Parerythropodium fulvum (Forskål) & 42 Acropora capillaris (Klunzinger) \\
\hline 8 Sarcophyton ehrenbergi v. Marenzeller & 43 Acropora corymbosa (Lamarck) \\
\hline 9 Sarcophyton elegans Moser & 44 Acropora cf. haimei \\
\hline 10 Sinularia candidula & (Milne Edwards \& Haime) \\
\hline Verseveldt \& Benayahu & 45 Acropora hemprichi (Ehrenberg) \\
\hline 11 Sinularia dactyloclados & 46 Acropora humilis (Dana) \\
\hline Verseveldt \& Benayahu & 47 Acropora hyacinthus (Dana) \\
\hline 12 Sinularia flabelliclavata & 48 Acropora pharaonis \\
\hline Verseveldt \& Benayahu & (Milne Edwards \& Haime) \\
\hline 13 Sinularia gardineri (Pratt) & 49 Acropora squarrosa (Ehrenberg) ${ }^{*}$ \\
\hline 14 Sinularia leptoclados (Ehrenberg) & 50 Acropora superba (Klunzinger) \\
\hline 15 Sinularia minima Verseveldt & 51 Acropora variabilis (Klunzinger) \\
\hline 16 Sinularia notanda Tixier-Durivault & 52 Acropora sp. \\
\hline 17 Sinularia polydactyla (Ehrenberg) & 53 Montipora effusa (Dana) \\
\hline 18 Sinularia querciformis (Pratt) & 54 Montipora ehrenbergi Verrill \\
\hline 19 Sinularia schuhmacheri & 55 Montipora granulosa Bernard \\
\hline Verseveldt \& Benayahu & 56 Montipora meandrina (Ehrenberg) \\
\hline Nephtheidae & 57 Montipora monasteriata (Forskål) \\
\hline 20 Nephthea laevis Kükenthal & 58 Montipora stilosa (Ehrenberg) \\
\hline 21 Dendronephthya hemprichi & 59 Montipora tuberculosa (Lamarck) \\
\hline (Klunzinger) & 60 Montipora venosa (Ehrenberg)" \\
\hline 22 Stereonephthya cundabiluensis & 61 Montipora verrucosa (Lamarck)* \\
\hline Verseveldt & 62 Montipora sp. \\
\hline 23 Lithophyton arboreum Kükenthal & Agariciidae \\
\hline 24 Paralemnalia eburnea Kükenthal & 63 Pavona clavus (Dana) \\
\hline 25 Paralemnalia thyrsoides (Ehrenberg) & 64 Pavona divaricata Lamarck \\
\hline Xeniidae & 65 Pavona explanulata (Lamarck) \\
\hline 26 Xenia macrospiculata Gohar & 66 Pavona maldivensis (Gardiner) \\
\hline 27 Xenia umbellata Lamarck & 67 Pavona varians Verrill \\
\hline 28 Heteroxenia fuscescens (Ehrenberg) & 68 Leptoseris mycetoseroides Wells \\
\hline 29 Anthelia fishelsoni Verseveldt & 69 Gardineroseris planulata (Dana) \\
\hline 30 Anthelia glauca Lamarck & Siderastreidae \\
\hline 31 Sympodium caeruleum Ehrenberg & 70 Coscinaraea monile (Forskål) \\
\hline Gorgonaria & Fungiidae \\
\hline Gorgoniidae & 71 Fungia echinata (Pallas) \\
\hline 32 Clathraria rubrinodis Gray & 72 Fungia fungites (Linnaeus) \\
\hline
\end{tabular}




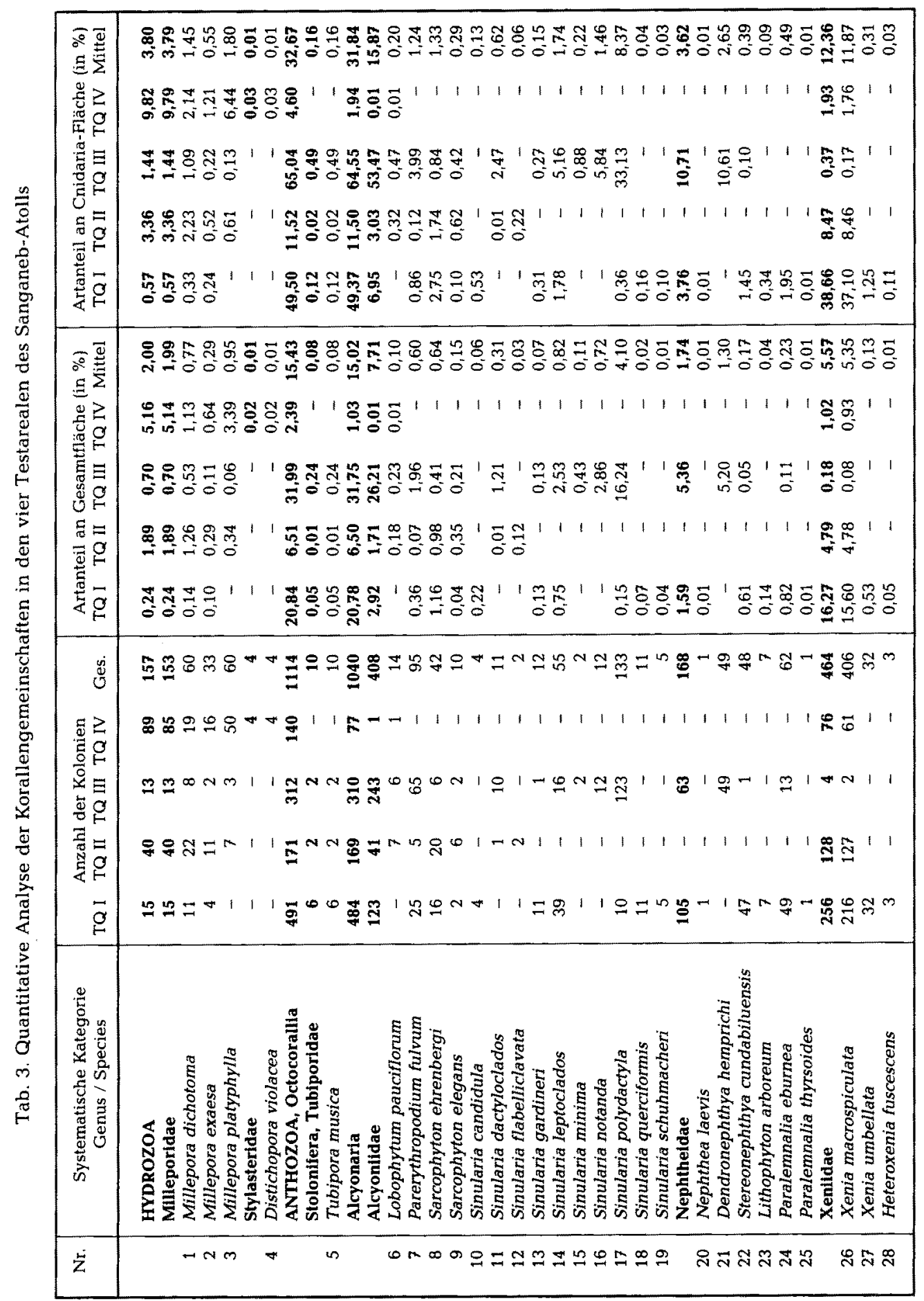




\begin{tabular}{|c|c|}
\hline 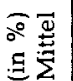 & 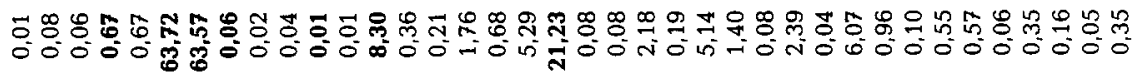 \\
\hline 营导 & 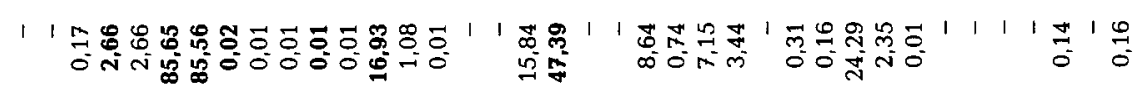 \\
\hline 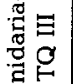 & 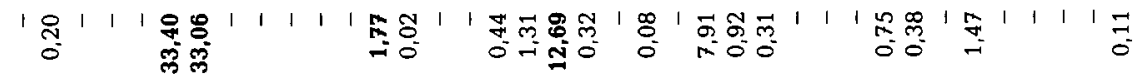 \\
\hline 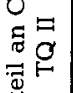 & 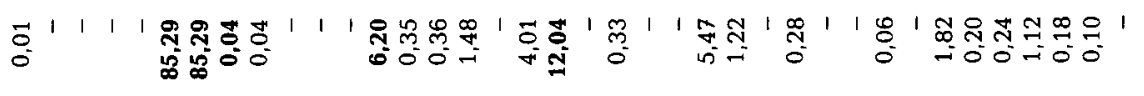 \\
\hline 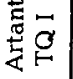 & 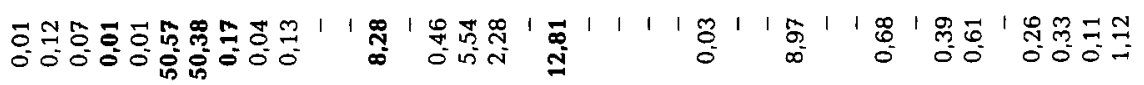 \\
\hline 尺导 & 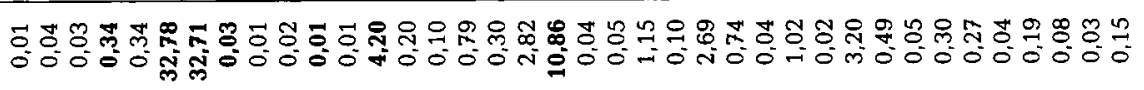 \\
\hline 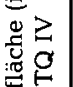 & 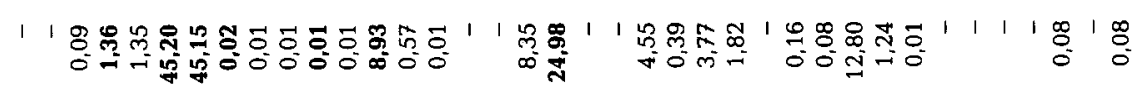 \\
\hline 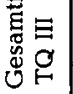 & 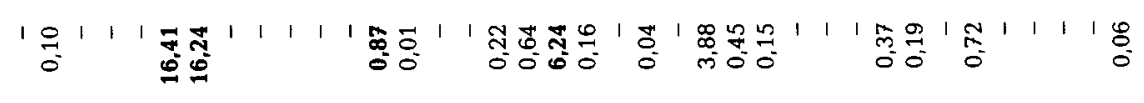 \\
\hline 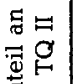 & 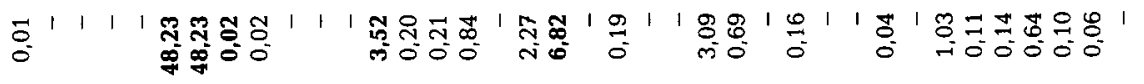 \\
\hline 焉 $\vec{g}$ & 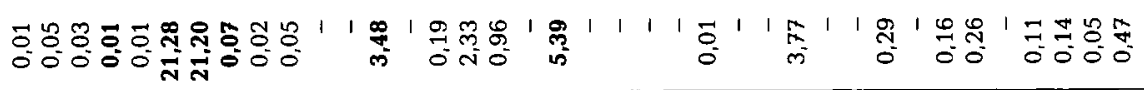 \\
\hline$\stackrel{\dot{d}}{0}$ & 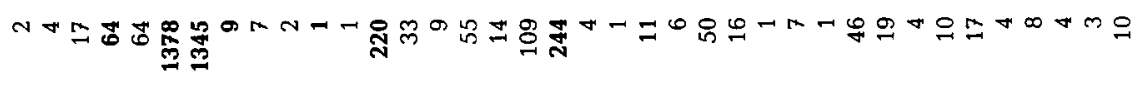 \\
\hline. & 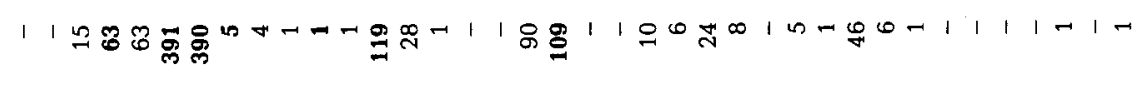 \\
\hline 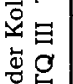 & 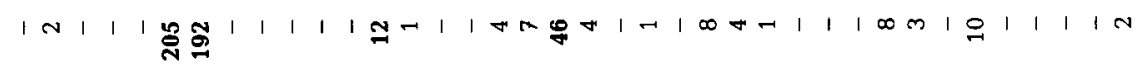 \\
\hline 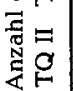 & 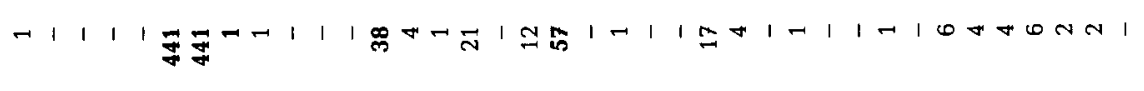 \\
\hline$\stackrel{\vec{\alpha}}{\stackrel{a}{*}}$ & 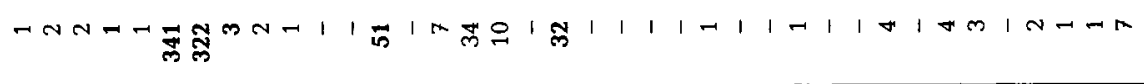 \\
\hline 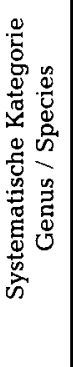 & 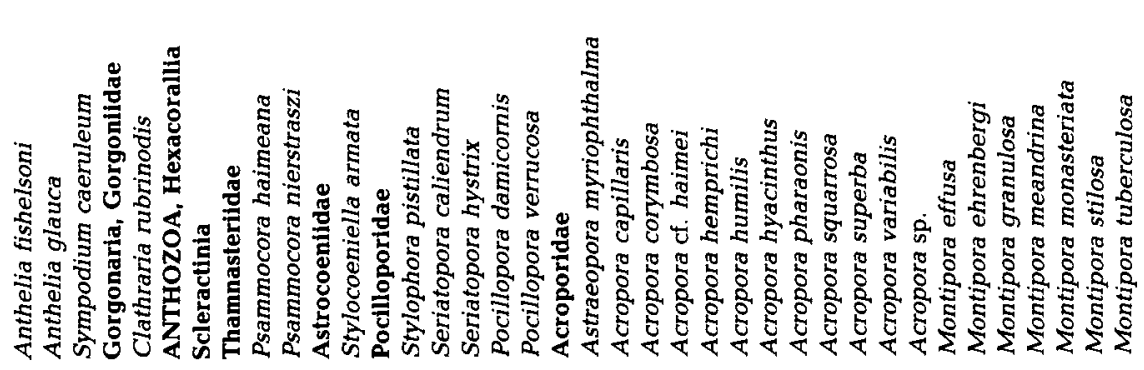 \\
\hline$\dot{\mathrm{z}}$ & 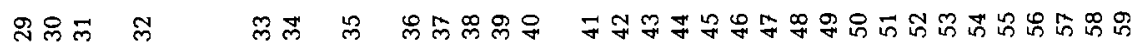 \\
\hline
\end{tabular}




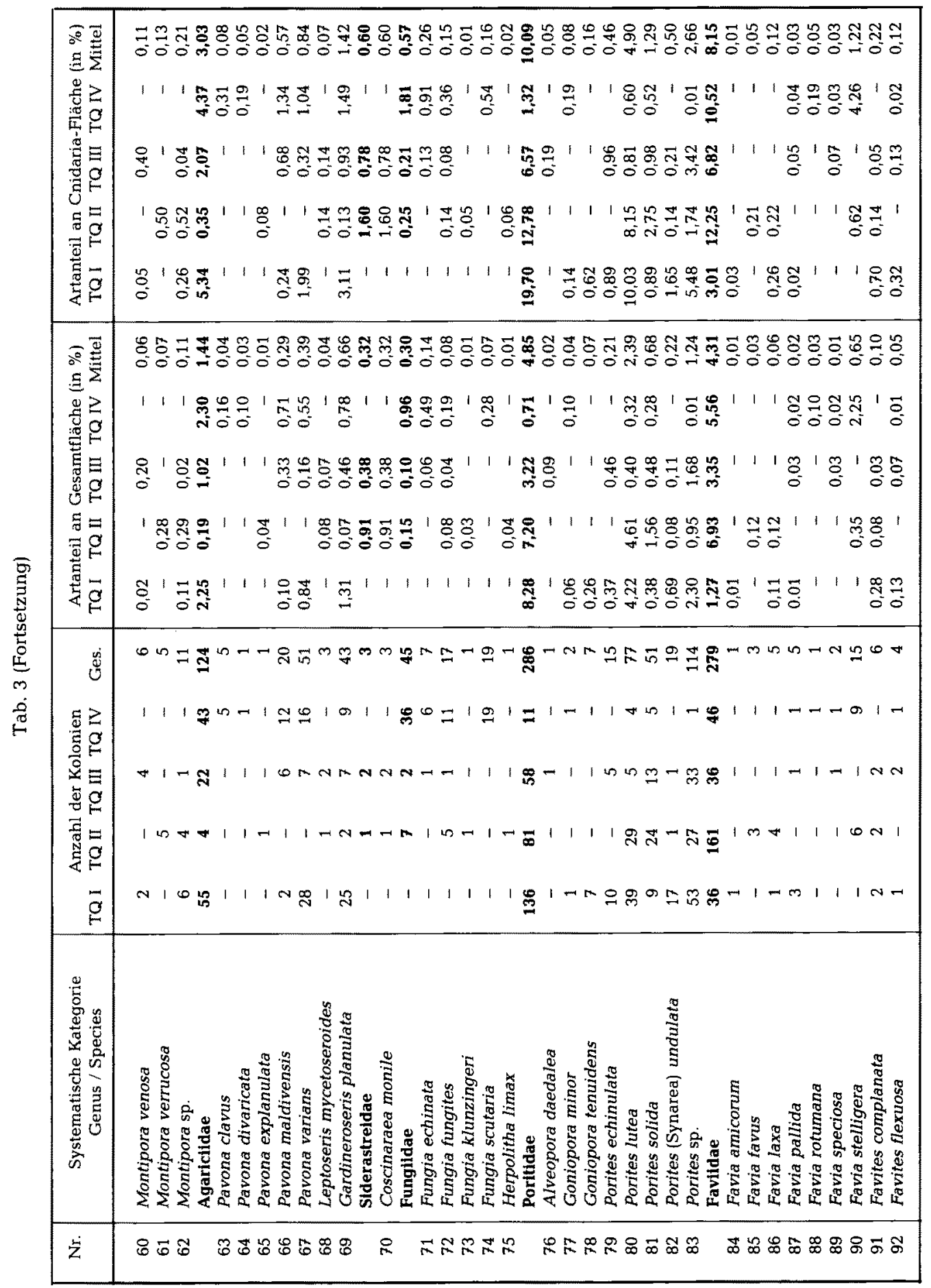




\begin{tabular}{|c|c|}
\hline 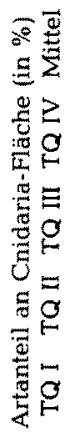 & 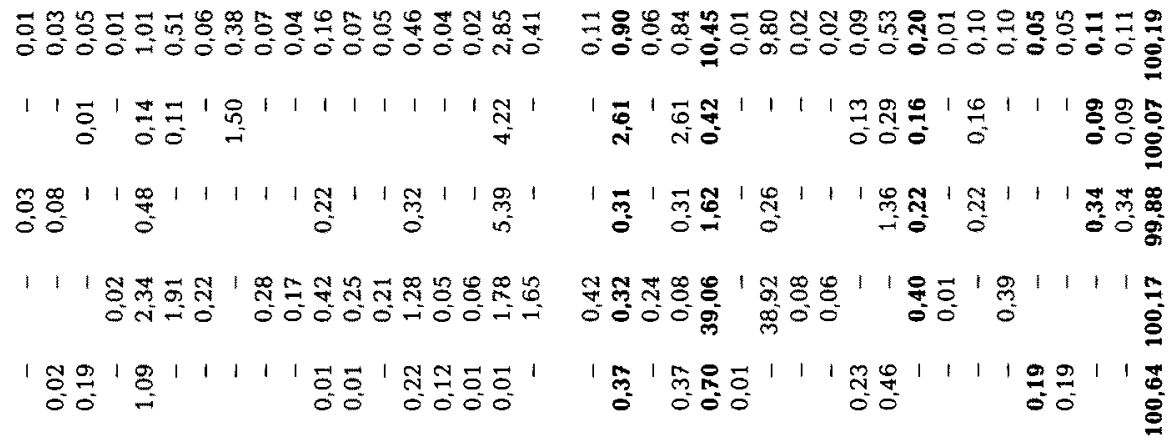 \\
\hline 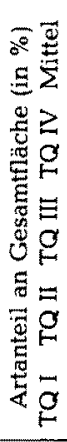 & 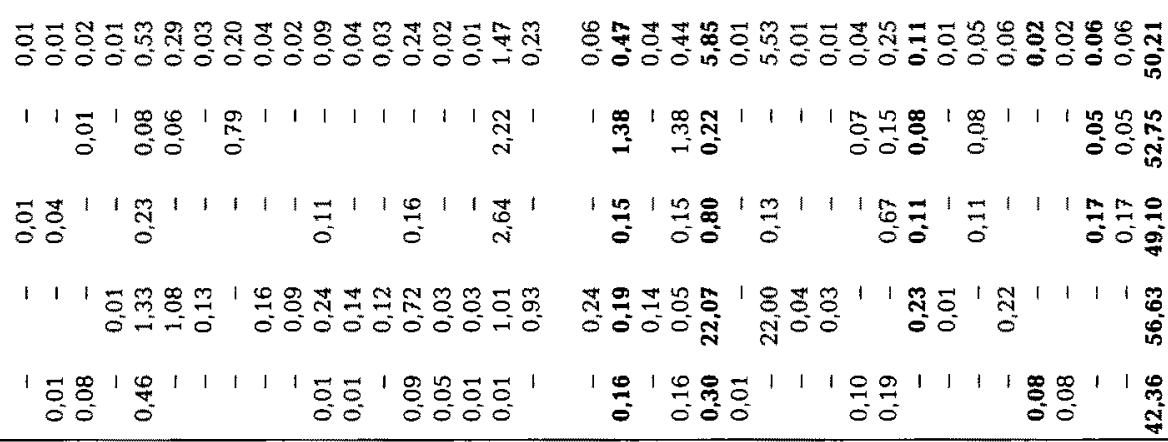 \\
\hline$\stackrel{\mathscr{J}}{\tilde{J}}$ & 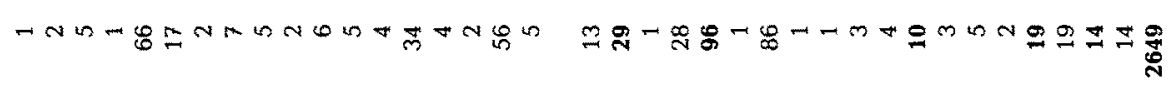 \\
\hline 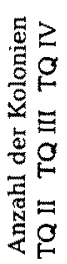 & 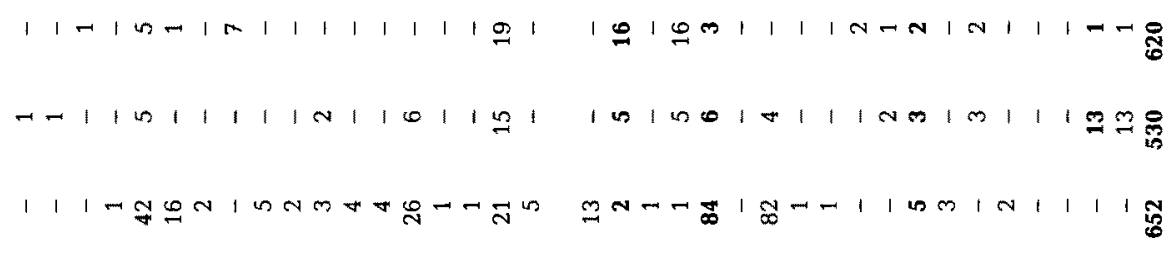 \\
\hline$\vec{g}$ & $10100+1|1+\pi| 1 \mid 10911 \frac{0}{0}$ \\
\hline 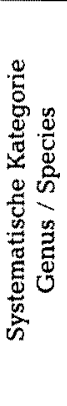 & 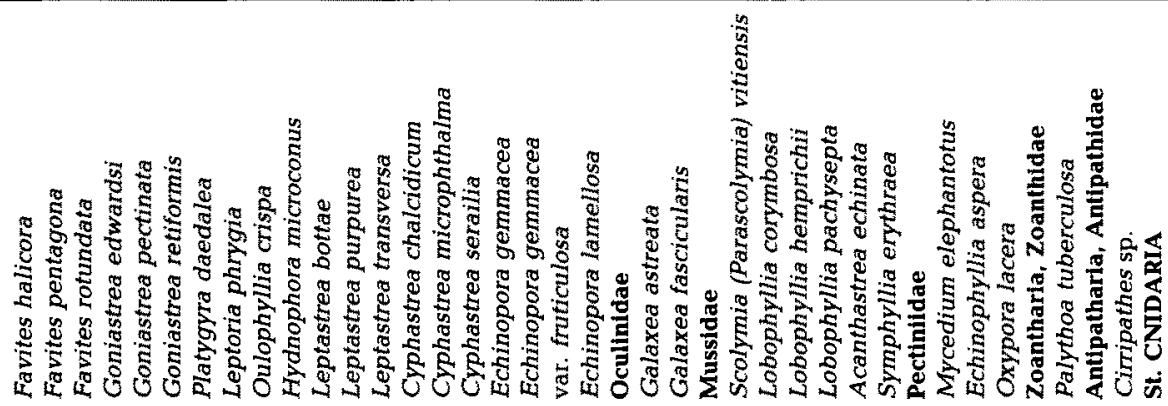 \\
\hline 之 & 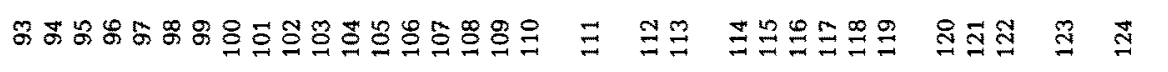 \\
\hline
\end{tabular}


Tab. 4. Besiedlung der vier Testareale des Sanganeb-Atolls durch Scleractinia, Alcyonaria und sonstige Cnidaria (* inkl. 1 Varietät)

\begin{tabular}{|c|c|c|c|c|c|c|c|}
\hline \multirow{2}{*}{$\begin{array}{l}\text { TQ- } \\
\text { Nr. }\end{array}$} & \multirow[t]{2}{*}{ Kategorie } & \multicolumn{3}{|c|}{ Anzahl der } & \multirow{2}{*}{$\begin{array}{l}\text { Bodenbe- } \\
\text { deckung } \\
\left(\text { in } \mathrm{m}^{2}\right)\end{array}$} & \multicolumn{2}{|c|}{ Prozentanteil von } \\
\hline & & Arten & $\begin{array}{l}\text { Gat- } \\
\text { tungen }\end{array}$ & $\begin{array}{l}\text { Kolo- } \\
\text { nien }\end{array}$ & & $\begin{array}{c}\text { Testareal- } \\
\text { Fläche }\end{array}$ & $\begin{array}{l}\text { Cnidaria- } \\
\text { Fläche }\end{array}$ \\
\hline \multirow[t]{3}{*}{ I } & Scleractinia & 44 & 19 & 322 & 5,29 & 21,2 & 50,4 \\
\hline & Alcyonaria & 20 & 11 & 484 & 5,19 & 20,8 & 49,4 \\
\hline & Sonstige & 5 & 4 & 41 & 0,09 & 0,4 & 0,9 \\
\hline \multirow[t]{3}{*}{ II } & Scleractinia* & 55 & 26 & 441 & 12,05 & 48,2 & 85,3 \\
\hline & Alcyonaria & 8 & 6 & 169 & 1,62 & 6,5 & 11,5 \\
\hline & Sonstige & 4 & 2 & 42 & 0,48 & 1,9 & 3,4 \\
\hline \multirow[t]{3}{*}{ III } & Scleractinia & 41 & 22 & 192 & 4,06 & 16,2 & 33,1 \\
\hline & Alcyonaria & 15 & 9 & 310 & 7,94 & 31,8 & 64,6 \\
\hline & Sonstige & 5 & 3 & 28 & 0,28 & 1,1 & 2,3 \\
\hline \multirow[t]{3}{*}{ IV } & Scleractinia & 43 & 21 & 390 & 11,25 & 45,2 & 85,6 \\
\hline & Alcyonaria & 3 & 3 & 77 & 0,26 & 1,0 & 1,9 \\
\hline & Sonstige & 6 & 4 & 153 & 1,64 & 6,6 & 12,6 \\
\hline
\end{tabular}

Tab. 5. Besiedlung der vier Testareale des Sanganeb-Atolls durch Kalkrotalgen (Co) und Poriferen (Po)

\begin{tabular}{|c|c|c|c|c|c|c|}
\hline \multirow[t]{2}{*}{ TQ-Nr. } & \multicolumn{2}{|c|}{$\begin{array}{l}\text { Anzahl der Kolonien } \\
\text { bzw. Bewuchsflächen }\end{array}$} & \multicolumn{2}{|c|}{$\begin{array}{l}\text { Bodenbedeckung } \\
\text { (in } \mathrm{m}^{2} \text { ) }\end{array}$} & \multicolumn{2}{|c|}{$\begin{array}{l}\text { Anteil an Gesamtfläche } \\
\text { (in \%) }\end{array}$} \\
\hline & $\mathrm{Co}$ & Po & Co & Po & $\mathrm{Co}$ & Po \\
\hline I & 38 & 87 & 0,84 & 0,31 & 3,4 & 1,2 \\
\hline II & 17 & 105 & 0,09 & 0,18 & 0,3 & 0.7 \\
\hline III & 20 & 214 & 0,53 & 0,25 & 2,1 & 1,0 \\
\hline IV & 53 & 149 & 0,47 & 0,13 & 1,9 & 0,5 \\
\hline
\end{tabular}

\section{Bodenbedeckung}

Insgesamt besteht die lebende Bodenbedeckung der vier Testareale aus 3337 Kolonien bzw. Bewuchsflächen, die sich gemäß Tabelle $6 a$ aufteilen.

Der Bodenbedeckung der Testareale mit lebenden Kolonien und Bewuchsflächen steht ihr Anteil an toten Substratflächen gegenüber. Hierhin gehören einerseits tote Korallenkolonien bzw. Kolonieteile, deren Artzugehörigkeit aber noch eindeutig zu erkennen ist, Korallenfelsflächen und grobes Korallenfelsgeröll, andererseits Sedimente wie abgebrochene Korallenästchen, Korallenfeinschutt, Korallensand und Kalkmulm. Für die unbelebten Substratflächen wurden die in Tabelle $6 \mathrm{~b}$ ausgewiesenen Anteile errechnet.

Die Gesamtarealbedeckung aus Lebendbewuchs und unbelebtem Substrat sollte pro Testareal $25 \mathrm{~m}^{2}$ Fläche und $100 \%$ ausmachen (Tab. 6c). Abweichungen davon müssen als Ungenauigkeit bei der Arealerfassung durch Ausmessen bzw. bei der Auswertung angesehen werden. 
Tab. 6. Lebendbewuchs und unbelebtes Substrat in den vier Testarealen des Sanganeb-Atolls

\begin{tabular}{|c|c|c|c|c|c|}
\hline \multirow[b]{2}{*}{ Bodenbedeckung } & \multicolumn{4}{|c|}{ Testquadrate } & \multirow[b]{2}{*}{ Mittelwert } \\
\hline & I & II & III & IV & \\
\hline \multicolumn{6}{|l|}{ (a) Lebendbewuchs: } \\
\hline Anzahl der Kolonien & 972 & 774 & 764 & 822 & 833 \\
\hline Bodenbedeckung $\left(\mathrm{m}^{2}\right)$ & 11,72 & 14,42 & 13,05 & 13,75 & 13,24 \\
\hline Prozent von Areal & 46,96 & 57,63 & 52,20 & 55,15 & 52,99 \\
\hline \multicolumn{6}{|l|}{ (b) Unbelebtes Substrat: } \\
\hline Bodenbedeckung $\left(\mathrm{m}^{2}\right)$ & 13,33 & 11,28 & 11,32 & 10,97 & 11,73 \\
\hline Prozent von Areal & 53,33 & 45,12 & 45,26 & 43,88 & 46,89 \\
\hline \multicolumn{6}{|l|}{$\begin{array}{l}\text { (c) Lebendbewuchs + un- } \\
\text { belebtes Substrat: }\end{array}$} \\
\hline Bodenbedeckung $\left(\mathrm{m}^{2}\right)$ & 25,05 & 25,70 & 24,37 & 24,72 & 24,97 \\
\hline Prozent von Areal & 100,29 & 102,75 & 97,46 & 99,03 & 99,88 \\
\hline
\end{tabular}

\section{Diversität}

Aus der vorgefundenen großen Zahl von Cnidaria-Arten und Kolonien leitet sich eine hohe Diversität her. Diese wurde für die vier Testquadrate in derselben Weise ermittelt, wie von Mergner \& Schuhmacher (1981) für ein Vorriffareal bei Aqaba. Ein Vergleich der Diversitätswerte vom Sanganeb-Atoll mit den sehr hohen Werten von Aqaba wird von Schuhmacher \& Mergner (1985) angestellt. Hier werden zunächst die Diversität und Eveness der vier Sanganeb-Quadrate angegeben (Tab. 7).

\section{Wuchsformen und Riffbildner}

Unter den riffbildenden Cnidaria lassen sich im wesentlichen drei Wuchsformen unterscheiden, nämlich verzweigte, krustenförmige und massige. $\mathrm{Zu}$ den sich verzweigenden Steinkorallen zählen u. a. alle Pocilloporidae und Acropora, zu den krustenför-

Tab. 7. Diversität (H') und Eveness (J) der Cnidaria-Gruppen in den vier Testarealen des SanganebAtolls

\begin{tabular}{|lccccc}
\hline & \multicolumn{7}{c|}{ Testquadrate } \\
Diversität & I & II & II & IV & Mittelwert \\
\hline Hydrocorallia & \multicolumn{7}{c}{ Diversität } \\
Octocorallia & 0,69 & 0,87 & 0,72 & 0,89 & 0,79 \\
Hexacorallia & 1,19 & 0,92 & 1,64 & 0,82 & 1,14 \\
Cridaria gesamt & 2,60 & 2,25 & 2,66 & 2,50 & 2,50 \\
& 2,34 & 2,55 & 2,64 & 2,77 & 2,58 \\
Hydrocorallia & 0,63 & 0,79 & 0,65 & 0,64 & 0,68 \\
Octocorallia & 0,39 & 0,42 & 0,61 & 0,59 & 0,50 \\
Hexacorallia & 0,70 & 0,57 & 0,74 & 0,66 & 0,67 \\
Cnidaria gesamt & 0,56 & 0,62 & 0,66 & 0,70 & 0,64 \\
\hline
\end{tabular}


mig wachsenden besonders Psammocora, Montipora, Pavona, Coscinaraea, Echinopora und die Pectiniidae und zu den massig wachsenden Gardineroseris, alle Poritidae, Faviidae (ohne Echinopora), Oculinidae und Mussidae (ohne Scolymia). Diesen Riffbildnern stehen als wichtigste Nichtriffbildner die Alcyonaria gegenüber. Im einzelnen verteilten sich die Wuchsformen in den vier Testarealen wie in Tabelle 8 angegeben.

Tab. 8. Wuchsformen, riffbildende und nicht-riffbildende Cnidaria in den vier Testarealen des Sanganeb-Atolls

\begin{tabular}{|c|c|c|c|c|c|c|}
\hline Besi & siedlungsstruktur in TQ-Nr.: & 1 & II & III & IV & Mittelwert \\
\hline \multirow[t]{5}{*}{ (a) } & Verzweigte Riffbildner: & & & & & \\
\hline & Anzahl der Kolonien & 68 & 96 & 48 & 299 & 128 \\
\hline & Flächenanteil $\left(\mathrm{m}^{2}\right)$ & 1,92 & 2,55 & 1,64 & 9,55 & 3,92 \\
\hline & Flächenanteil (\%) & 7,7 & 10,2 & 6,5 & 38,3 & 15,7 \\
\hline & Anteil an Cnidaria (\%) & 18,8 & 18,1 & 13,3 & 72,7 & 30,7 \\
\hline \multirow{5}{*}{ (b) } & Massige Riffbildner: & & & & & \\
\hline & Anzahl der Kolonien & 210 & 293 & 99 & 66 & 167 \\
\hline & Flächenanteil $\left(\mathrm{m}^{2}\right)$ & 2,83 & 8,59 & 1,39 & 1,60 & 3,60 \\
\hline & Flächenanteil $(\%)$ & 11,4 & 34,3 & 5,6 & 6,3 & 14,4 \\
\hline & Anteil an Cnidaria (\%) & 27,0 & 60,7 & 10,4 & 12,2 & 27,6 \\
\hline \multirow{5}{*}{ (c) } & Krustenförmige Riffbildner: & & & & & \\
\hline & Anzahl der Kolonien & 84 & 94 & 60 & 115 & 88 \\
\hline & Flächenanteil $\left(\mathrm{m}^{2}\right)$ & 0,65 & 1,40 & 1,27 & 1,41 & 1,19 \\
\hline & Flächenanteil (\%) & 2,5 & 5,6 & 5,1 & 5,6 & 4,7 \\
\hline & Anteil an Cnidaria (\%) & 5,6 & 9,9 & 10,3 & 10,6 & 9,1 \\
\hline \multirow{5}{*}{ (d) } & Riffbildende Cnidaria: & & & & & \\
\hline & Anzahl der Kolonien & 362 & 483 & 207 & 480 & 383 \\
\hline & Flächenanteil $\left(\mathrm{m}^{2}\right)$ & 5,40 & 12,54 & 4,30 & 12,56 & 8,70 \\
\hline & Flächenanteil (\%) & 21,6 & 50,1 & 17,2 & 50,2 & 34,8 \\
\hline & Anteil an Cnidaria (\%) & 51,7 & 88,7 & 35,0 & 95,5 & 67,4 \\
\hline \multirow{5}{*}{ (e) } & Nicht-riffbildende Cnidaria: & & & & & \\
\hline & Anzahl der Kolonien & 485 & 169 & 323 & 140 & 279 \\
\hline & Flächenanteil $\left(\mathrm{m}^{2}\right)$ & 5,20 & 1,62 & 7,98 & 0,61 & 3,85 \\
\hline & Flächenanteil (\%) & 20,8 & 6,5 & 31,9 & 2,4 & 15,4 \\
\hline & Anteil an Cnidaria (\%) & 49,4 & 11,5 & 64,9 & 4,7 & 32,6 \\
\hline
\end{tabular}

Über den Zusammenhang zwischen Wuchsformenverteilung einerseits und den Einzelfaktoren der Wasserbewegung anderseits wird an späterer Stelle noch berichtet.

\section{Bestandsbildner, Leit- und Indikatorarten, biophysiographische Zonen}

Das Hervortreten von Leitarten durch quantitatives oder (und) optisches Überwiegen über andere Arten des gleichen Areals und die Bildung biophysiographischer Zonen durch lokal umschriebene Bestände dieser Leitarten wurden ermittelt, indem aus Tabelle 3 die Arten mit den höchsten Koloniezahlen pro Testareal und der größten Flächenbedeckung in $\mathrm{m}^{2}$, vor allem aber mit den größten Prozentanteilen an der durch Cnidaria besiedelten Fläche herausgezogen werden. Die hierbei gewonnene Artenauswahl (Tab. 9) tritt auch optisch in den Abbildungen 12-15 hervor. 
Tab. 9. Bestandsbildende Cnidaria-Arten in den vier Testarealen des Sanganeb-Atolls (mit Artnummern von Tabelle 2)

\begin{tabular}{|clrrr|}
\hline TQ- & Gattung, Art (Art-Nr.) & $\begin{array}{c}\text { Kolonie- } \\
\text { anzahl }\end{array}$ & $\begin{array}{c}\text { Boden- } \\
\text { bedeckung } \\
\left(\mathrm{m}^{2}\right)\end{array}$ & $\begin{array}{r}\% \text {-Anteil von } \\
\text { Cnidaria- } \\
\text { Fläche }\end{array}$ \\
\hline \multirow{2}{*}{ I } & Xenia macrospiculata (26) & 216 & 3,90 & 37,1 \\
& Porites lutea (80) & 39 & 1,05 & 10,0 \\
II & Xenia macrospiculata (26) & 127 & 1,20 & 8,5 \\
& Acropora hemprichi (45) & 17 & 0,77 & 5,5 \\
& Porites lutea (80) & 29 & 1,15 & 8,2 \\
& Lobophyllia corymbosa (115) & 82 & 5,50 & 38,9 \\
III & Sinularia leptoclados (14) & 16 & 0,63 & 5,2 \\
& Sinularia notanda (16) & 12 & 0,72 & 5,8 \\
& Sinularia polydactyla (17) & 123 & 4,06 & 33,1 \\
& Dendronephthya hemprichi (21) & 49 & 1,30 & 10,6 \\
& Acropora hemprichi (45) & 8 & 0,97 & 7,9 \\
& Echinopora gemmacea (109) & 15 & 0,66 & 5,4 \\
IV & Millepora platyphylla (3) & 50 & 0,85 & 6,4 \\
& Pocillopora verrucosa (40) & 90 & 2,09 & 15,8 \\
& Acropora corymbosa (43) & 10 & 1,14 & 8,6 \\
& Acropora hemprichi (45) & 24 & 0,94 & 7,2 \\
& Acropora superba (50) & 46 & 3,20 & 24,3 \\
& Echinopora gemmacea (109) & 19 & 0,56 & 4,2 \\
\hline
\end{tabular}

Einige Arten bilden also Bestände in mehreren Testarealen, wie etwa Acropora hemprichi in 3 Testquadraten sowie Xenia macrospiculata, Porites lutea und Echinopora gemmacea in je 2. Außer den oben aufgeführten 13 bestandsbildenden Arten können noch einige andere erwähnt werden, die in größerer Koloniezahl auftreten oder kleinere Arealflächen bedecken. Doch bilden sie keine Bestände wie die vorgenannten Arten. Es sind: Millepora dichotoma (1) in den Testarealen II und IV, Parerythropodium fulvum (7) in Testareal III, Seriatopora hystrix (38) in Testareal I, Porites sp. (83) in Testareal I sowie Favia stelligera (90) in Testareal IV.

Als Leitarten mit hohen Prozentanteilen an der gesamten Siedlungsfläche der Cnidaria treten in den einzelnen Testarealen hervor: TQI: Xenia macrospiculata $(+)$ mit über $37 \%$, Porites lutea mit $10 \%$; TQ II: Lobophyllia corymbosa (t) mit fast $39 \%$, Porites lutea mit über $8 \%$; TQ III: Sinularia polydactyla (+) mit über $33 \%$, Dendronephthya hemprichi mit fast $11 \%$; TQIV: Acropora superba (+) mit über $24 \%$, Pocillopora verrucosa mit fast $16 \%$.

In jedem der vier Testareale dominiert also e ine Art $(+)$ eindeutig über alle anderen, und zwei Arten bedecken zusammen stets schon über $40 \%$ der gesamten von Cnidariern besiedelten Arealfläche, obwohl doch in dieser zwischen 51 und 68 Arten vorkommen.

Inwieweit die Leitarten auch Indikatorarten für bestimmte Umweltbedingungen darstellen, etwa für die Häufigkeit des Wasseraustausches oder gewisser Wasserbewegungsformen, soll später noch untersucht werden. Auffallend ist zumindest, daß in 


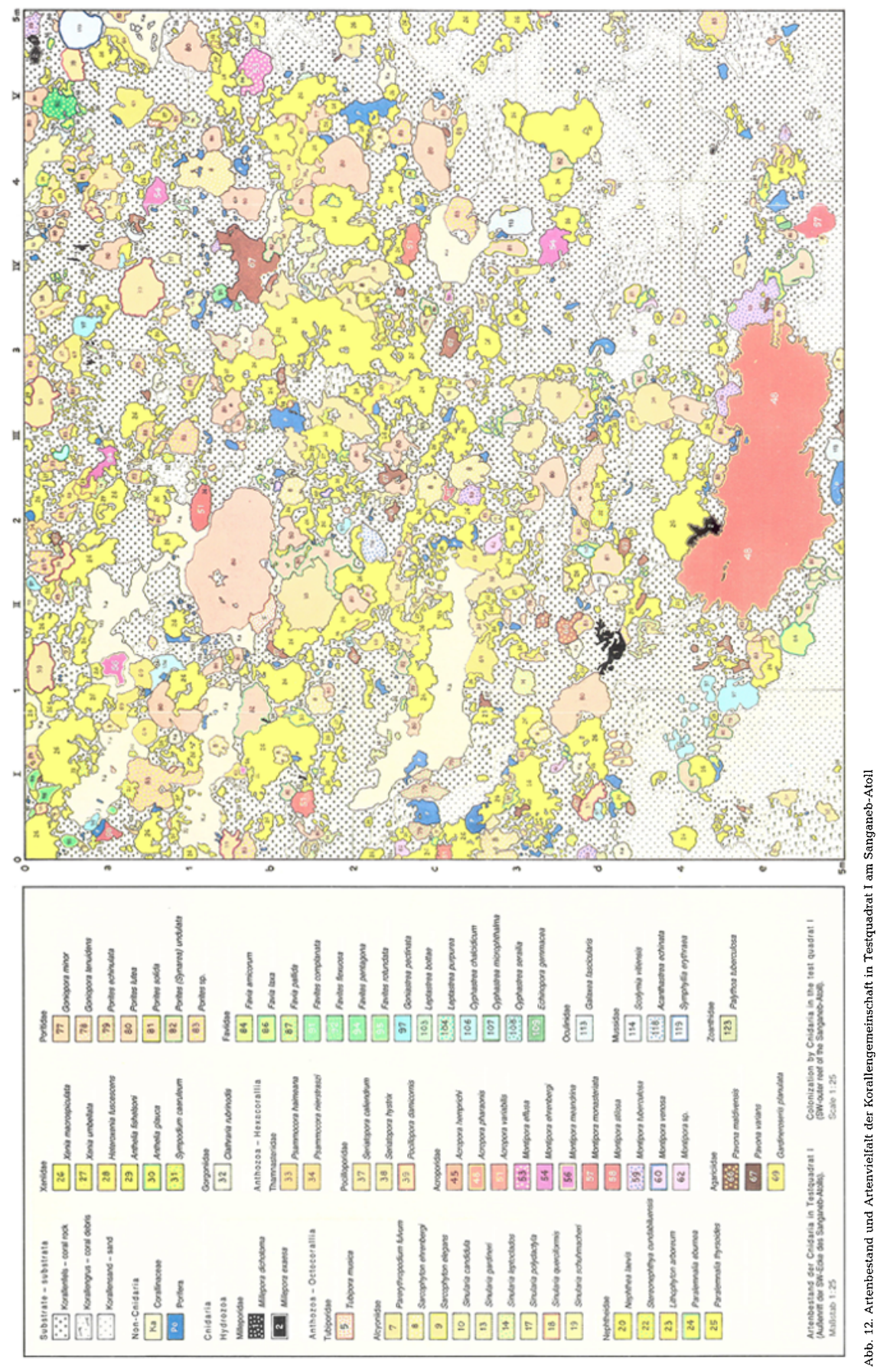



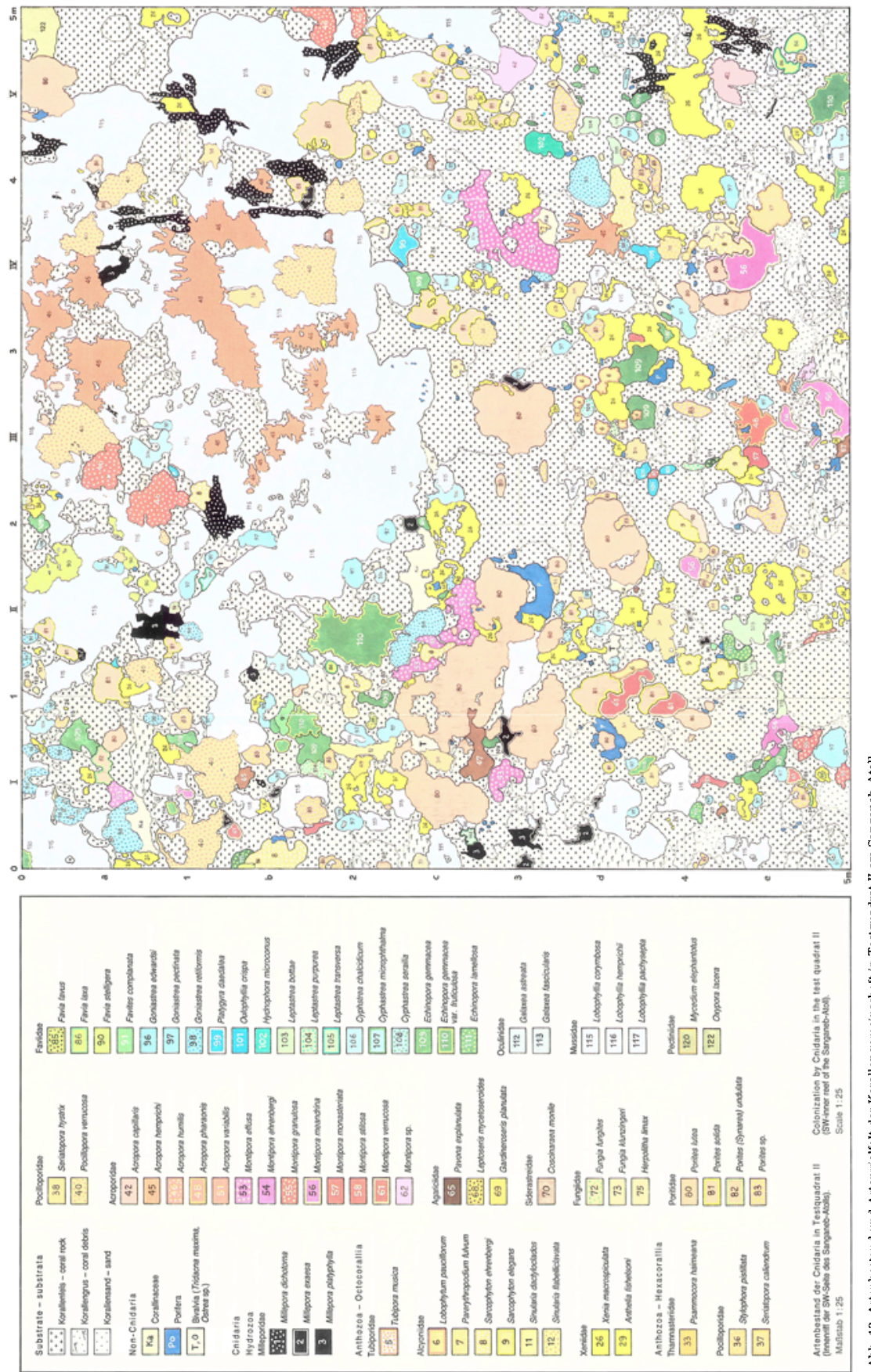


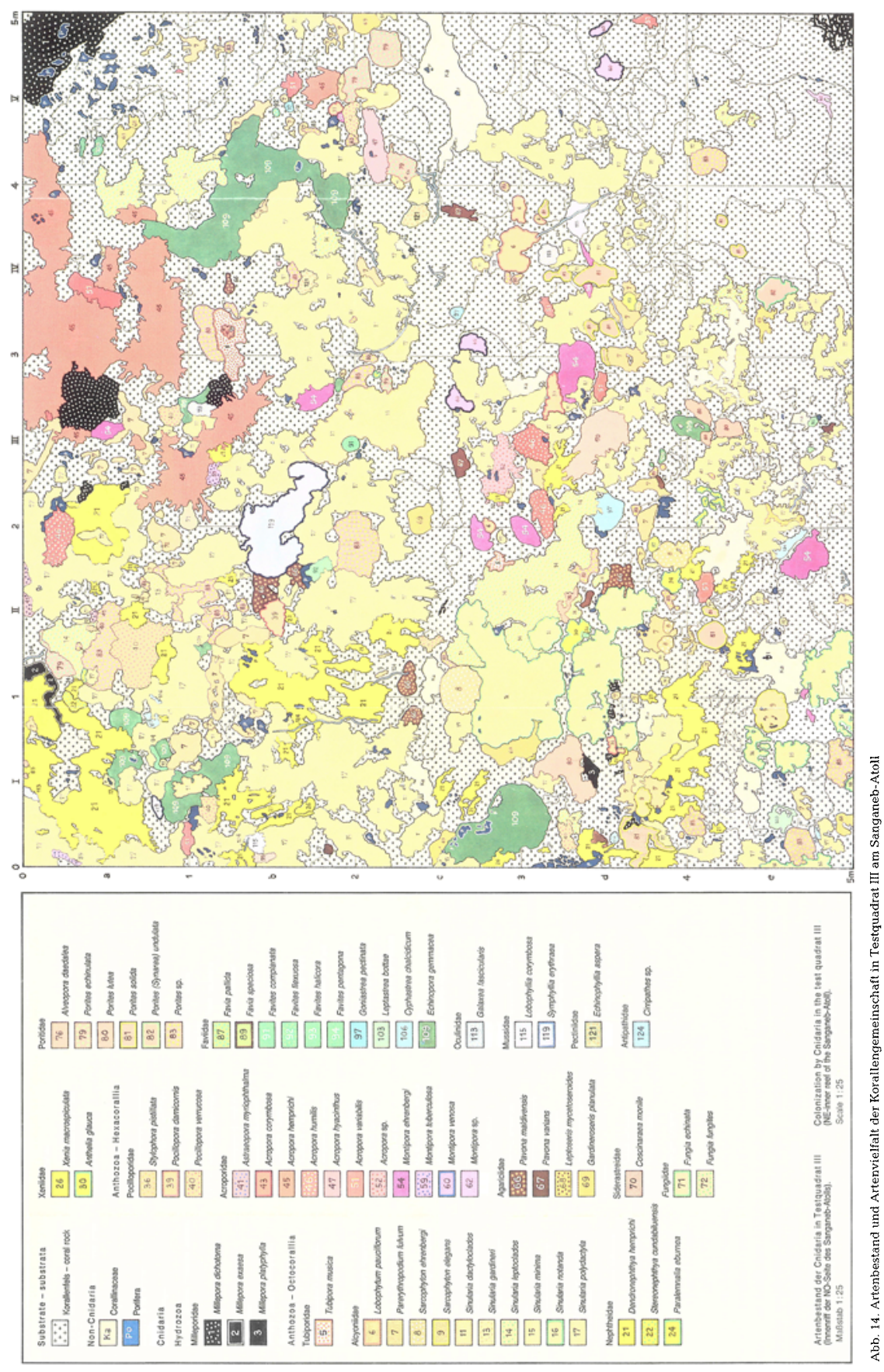




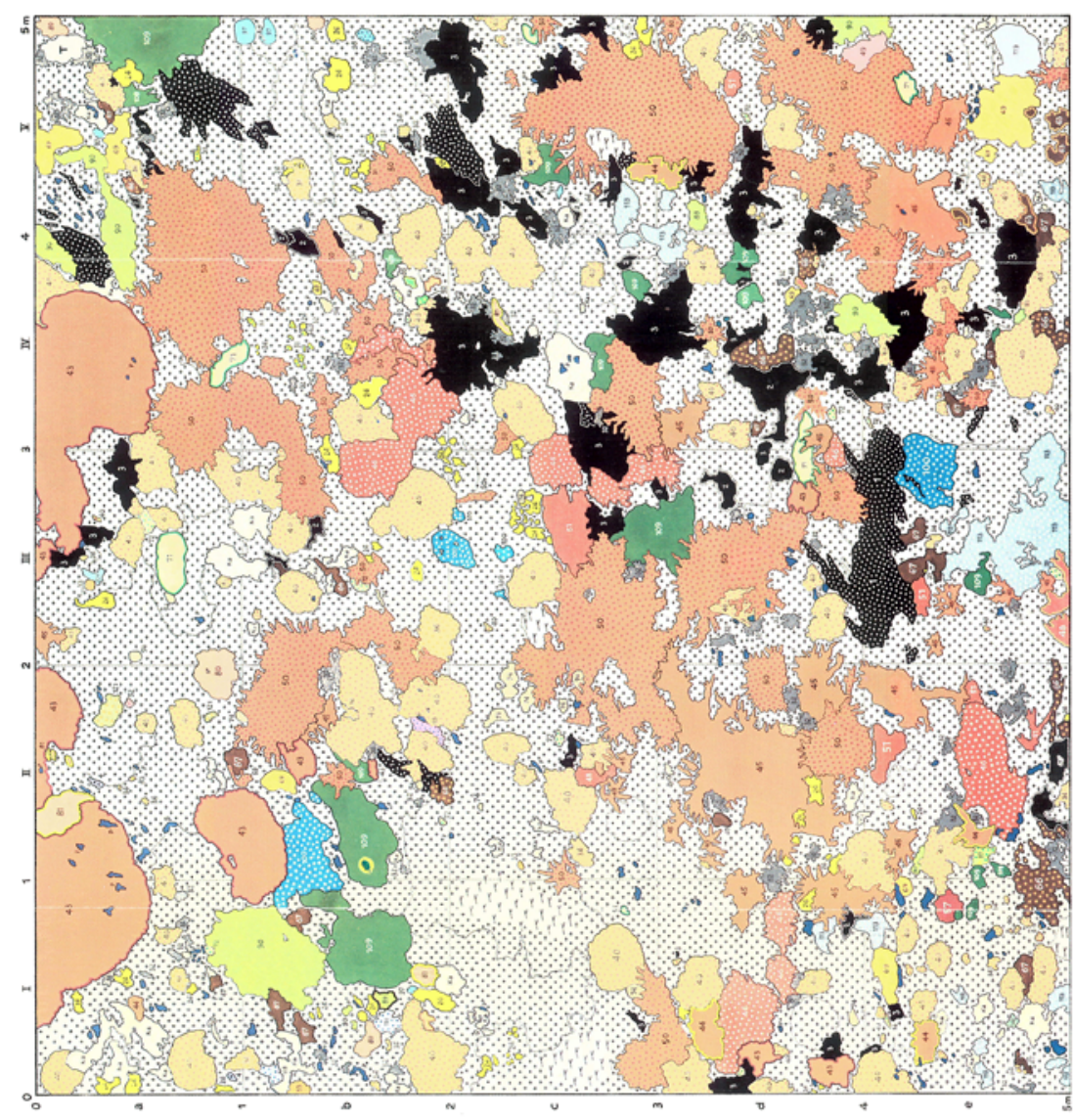

after page 404

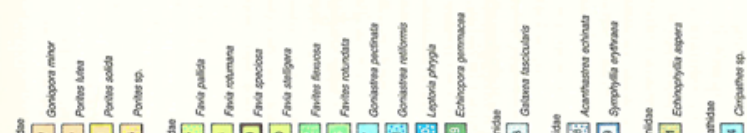

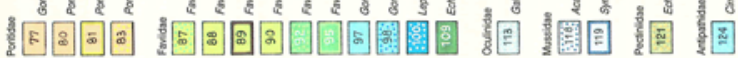

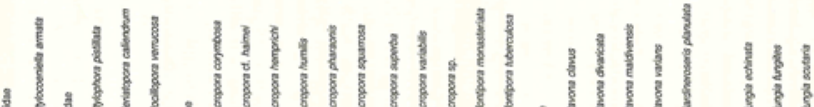

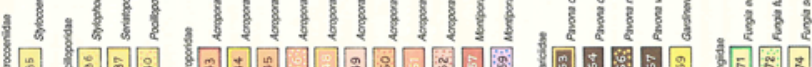

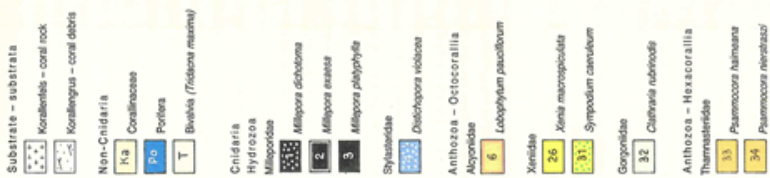


jedem einzelnen Testareal, d.h. auf jedem der vier Riffhänge des Sanganeb-Atolls, andere Leitarten bzw. -kombinationen auftreten.

Schließlich ist zu prüfen, ob die Leitarten in den einzelnen Testarealen auch bestimmte biophysiographische Zonen charakterisieren und ob diese die ganze Arealfläche oder nur Teilbereiche davon erfassen (Tab. 9).

In Testa real I (Abb. 12) bildet Xenia macrospiculata mit ihren 216 Kolonien und $37,1 \%$ Anteil an der von Cnidariern bedeckten Arealfläche die unbestrittene Leitart. Doch sind ihre niedrig-rasenwüchsigen Bestände nur locker und unregelmäßig über die ganze Arealfläche verteilt und fügen sich auch optisch nicht zu einer klar umschriebenen Zone zusammen. Vornehmlich in der unteren Arealhälfte finden sich 5 Porites-Arten ( $P$. echinulata, $P$. Iutea, $P$. solida, $P$. undulata und $P$. sp.) mit zusammen 128 Kolonien und 18,9\% Anteil an der Cnidaria-Fläche. Sie könnten - aber nur mit Vorbehalt - eine freilich stark mit Xenia macrospiculata durchsetzte Porites-Zone charakterisieren.

In Testareal I (Abb. 13) stellt Lobophyllia corymbosa mit 82 meist großen Kolonien und 38,9\% Anteil an der Cnidaria-Fläche eindeutig die Leitart und bildet auch in der oberen Arealhälfte eine geschlossene biophysiographische Zone gleichen Namens. In der unteren Hälfte bestimmen dagegen wieder 3 Porites-Arten ( $P$. lutea, $P$. solida und $P$. sp.) die Zonierung und bilden mit 80 Kolonien und $12,6 \%$ Anteil an der Cnidaria-Fläche eine - wenn auch nicht eindeutig charakterisierte und klar umschriebene - Porites-Zone.

Leitarten des T e sta r e a ls III (Abb. 14) sind Sinularia polydactyla und Dendronephthya hemprichi mit zusammen 172 Kolonien und 43,6\% Anteil an der von Cnidariern besiedelten Arealfläche. Wegen der starken Untermischung von $S$. polydactyla mit äußerlich ähnlichen anderen Sinularia-Arten ( $S$. dactyloclados, $S$. leptoclados und $S$. notanda) mit zusammen 38 Kolonien und 13,5\% Flächenanteil sollte jedoch nur allgemein von einer Sinularia-Dendronephthya hemprichi-Zone gesprochen werden. Sie tritt vor allem im linken (nordwestlichen) oberen und mittleren Tiefenbereich des Areals hervor.

In $\mathrm{T}$ e sta rea l IV (Abb. 15) stellen die beiden Leitarten Acropora superba und Pocillopora verrucosa mit zusammen 136 Kolonien 40,1\% der Cnidaria-Fläche. Zu ihnen gesellen sich 8 andere, teilweise ähnliche Acropora-Arten (A. corymbosa, cf. haimei, hemprichi, humilis, pharaonis, squarrosa, variabilis und sp.) mit zusammen 61 Kolonien und 22,8\% Flächenanteil. Daher sollte diese biophysiographische Zone als AcroporaPocillopora verrucosa-Zone bezeichnet werden.

\section{DISKUSSION}

Wie in zahlreichen Untersuchungen nachgewiesen, darunter bei Wood-Jones (1910), Marshall (1931), Hiatt (1957), Bradbury \& Young (1981) und speziell für das Rote Meer bei Mergner (1967) und später ausführlich bei Mergner \& Schuhmacher (1974), spielen Lage und Ausrichtung eines Riffareals zur vorherrschenden Wasserbewegung eine bedeutende Rolle für seine Besiedlungsstruktur. Vor allem werden durch die Intensität der Wasserbewegung (Häufigkeit des Wasseraustausches pro Volumen- und Zeiteinheit) und ihre Formen (Brandung, Restwellen, Rücklauf, Vertikaloszillation, Rifflängsströmung und Horizontaloszillation) sowie durch beider Kombination entschei- 
dende Einflüsse auf die jeweilige Riffbiozönose ausgeübt. Sie betreffen ihre Artenzusammensetzung, ihre Diversität, ihre horizontale und - zusammen mit Einflüssen der Beleuchtung - ihre vertikale Zonierung sowie ihre kurz- und langfristigen Veränderungen im Gefolge des Auf-, Um- und Abbaues des Riffs. Bei Berücksichtigung dieser Erkenntnisse durch Auswahl möglichst genau gegen die Hauptwasserbewegungsrichtung gelegener Untersuchungsareale sind also auch entsprechend differenzierte Beobachtungsergebnisse zur Besiedlungsstruktur zu erwarten.

Als besonders geeignet für eine vergleichende Diskussion bieten sich die Korallengemeinschaften der vier Riffhänge eines Atollprofiles an, wenn dieses der genannten Hauptrichtung folgt. Hierzu können nunmehr die Ergebnisse der strukturellen und der quantitativen Analyse der Korallengemeinschaften in den vier Testarealen entlang eines SSW-NNO-Profiles durch das Sanganeb-Atoll herangezogen werden. Dabei soll die Frage im Vordergrund stehen, inwieweit die Wasserbewegung als Verursacher bestimmter Besiedlungsstrukturen nachzuweisen ist.

Schon bei kurzer Beurteilung lassen sich je zwei Testquadrate zusammenordnen, nämlich TQ II und TQ IV sowie TQ I und TQ III. Die beiden mit ihrer Fläche gegen Nordnordosten und damit gegen die gemittelte Hauptrichtung der Wasserbewegung gestellten Testquadrate II und IV fallen sofort durch das eindeutige Überwiegen der charakteristischen Hartstrukturen riffbildender Cnidaria gegenüber den Weichkorallen auf. So treten auf den zeichnerischen Strukturdarstellungen (Abb. 8 und 10) wie auch auf den farbigen Bestandsplänen (Abb. 13 und 15) bestimmte Hydrokorallen und Steinkorallen als Leitarten schon optisch hervor und die riffbildenden Cnidaria insgesamt als dominierendes Element. Die quantitative Analyse bestätigt diesen Eindruck:

In TQII stellen 441 Kolonien von 54 Scleractinia-Arten auf $12,05 \mathrm{~m}^{2}$ Bodenfläche $85,3 \%$ aller hier siedelnder Cnidaria, während nur $11,5 \%$ den 169 Kolonien von 8 Alcyonaria-Arten mit $1,62 \mathrm{~m}^{2}$ Fläche zuzurechnen sind. Leitart ist Lobophyllia corymbosa mit fast $39 \%$.

In TQIV bedecken 390 Kolonien von 42 Scleractinia-Arten $11,25 \mathrm{~m}^{2}$ Fläche und stellen auch $85,6 \%$ aller Cnidaria. Demgegenüber spielen nur 3 Alcyonaria-Arten mit 77 Kolonien auf $0,26 \mathrm{~m}^{2}$ Fläche und einem Anteil von nur 1,9\% des Cnidaria-Bestandes kaum eine Rolle. Als Leitarten sind hier Acropora superba mit über $24 \%$ und Pocillopora verrucosa mit fast $16 \%$ Anteil zu nennen.

Ganz anders liegen die Strukturverhältnisse in den beiden gegen SSW ausgerichteten und von der Hauptrichtung der Wasserbewegung abgewandten Testarealen I und III. Weichkorallen bestimmen schon optisch beim Betrachten der Strukturzeichnungen (Abb. 7 und 9) und farbigen Pläne (Abb, 12 und 14) das Bild der Cnidariergemeinschaft. Dieser Eindruck wird durch die Ergebnisse der quantitativen Analyse unterstrichen:

In TQ I sind die Anteile beider Cnidaria-Ordnungen weitgehend ausgeglichen. Hier stellen nämlich 20 Alcyonaria-Arten mit 484 Kolonien auf $5,19 \mathrm{~m}^{2}$ Bodenfläche $49,4 \%$ aller Cnidaria, während der Anteil von 43 Scleractinia-Arten mit 322 Kolonien auf $5,29 \mathrm{~m}^{2}$ Fläche $50,4 \%$ ihres Bestandes erreicht. Leitart ist mit über $37 \%$ die Alcyonarie Xenia macrospiculata.

In TQIII überwiegen jedoch die Weichkorallen eindeutig. So stehen den 15 Alcyonaria-Arten mit 310 Kolonien auf fast $8 \mathrm{~m}^{2}$ Bodenfläche und einem Anteil von $64,6 \%$ des Cnidaria-Bestandes nur 33,1\% von 39 Scleractinia-Arten mit 192 Kolonien auf $4,06 \mathrm{~m}^{2}$ Fläche gegenüber. Leitarten sind die beiden Alcyonarien Sinularia polydactyla mit über 
$33 \%$ aller von Cnidariern besiedelten Flächen und Dendronephthya hemprichi mit fast $11 \%$.

Insgesamt unterscheiden sich also jeweils zwei Testareale mit einem überwiegenden Anteil von Steinkorallen von zwei anderen mit einem solchen von Weichkorallen. Die Ursache für den Unterschied muß zunächst bei den maßgeblichen abiotischen Faktoren gesucht werden: Beleuchtung und Wasserbewegung. In einer Wassertiefe von 5 bis $10 \mathrm{~m}$ erbringen zooxanthellenhaltige Korallen ihre höchste Produktivität im Vertikalvergleich (z. B. Barnes \& Taylor, 1973; Mergner \& Svoboda, 1977; Svoboda, 1978). Selbst unter Berücksichtigung dessen, daß die Beleuchtung an den vier Testquadraten leicht differiert - die beiden nach NO gerichteten TQII und TQIV erhalten bei niedrigem Sonnenstand mehr streifendes Licht, die beiden nach SW gerichteten TQI und TQ III hingegen liegen steiler, was vor allem bei dem teilweise überhängenden TQ III bei hohem Sonnenstand zu einem ausgeprägten Licht-Schatten-Muster führt -, ist aus diesen Unterschieden allein keinesfalls die differierende Besiedlung abzuleiten. Mit Ausnahme von Dendronephthya hemprichi sind im übrigen alle bestandsbildenden Hydro-, Stein- und Weichkorallen mit Zooxanthellen assoziiert und daher prinzipiell gleichermaßen auf Licht angewiesen.

Hieraus ist zu schließen, daß die entscheidenden Einflußnahmen auf Artenzusammensetzung und Bestandsbildung in den vier Testarealen von der Wasserbewegung (und den hiervon abhängenden Parametern Temperatur, Sauerstoffgehalt, Planktonzufuhr und Sedimentation) ausgehen.

Das an der Außenseite des Sanganeb-Ostriffes gelegene T e stareal IV ist am stärksten gegen den vorherrschenden Seegang aus Nordost exponiert. Dieser war im Untersuchungszeitraum mit einer durchschnittlichen Mindesthöhe von 1,1 m stärker als in allen anderen Testarealen. Dem entspricht auch die Besiedlungsstruktur der hier vorgefundenen Korallengemeinschaft: Wie an allen ähnlich stark exponierten Riffabschnitten in der Region um Port Sudan, etwa nach den Beobachtungen von Mergner in den Jahren 1975 und 1979 an der Nordostfront des North Towartit-Barriereriffes und des Wingate-Barriereriffes, überwiegen auch in Areal IV verzweigte Wuchsformen.

Unter den riffbildenden Cnidaria nehmen sie mit fast 300 Kolonien (von 480) auf $9,55 \mathrm{~m}^{2}$ Bodenfläche (von 12,56 $\mathrm{m}^{2}$ ) einen Anteil von $72,7 \%$ ein (von $95,5 \%$ ). Demgegenüber stellen nicht-riffbildende Cnidaria nur 140 Kolonien auf $0,61 \mathrm{~m}^{2}$ mit einem Anteil von $4,7 \%$. Unter den verzweigten Steinkorallen fallen wiederum die großen Bestände geweihförmig wachsender Acropora-Arten auf (Abb. 6c, 10,11h, 15). Allein A. hemprichi und $A$. superba bilden zusammen 70 Kolonien auf $4,14 \mathrm{~m}^{2}$ Bodenfläche mit einem Anteil von 31,5\% aller Cnidaria dieses Areals. Untermischt sind die Acropora-Bestände mit nicht weniger als 90 Kolonien von Pocillopora verrucosa auf $2,1 \mathrm{~m}^{2}$ mit einem Anteil von $15,8 \%$ der Cnidaria (Abb. 11g). Aber auch die verzweigten Millepora dichotoma und $M$. platyphylla spielen mit zusammen 69 Kolonien auf $1,13 \mathrm{~m}^{2}$ Fläche und $8,6 \%$ Anteil noch eine wichtige Rolle.

Hinzuweisen ist auch auf eine Besonderheit des Steinkorallenbestandes in TestarealIV (Tab. 10): Von insgesamt 11 am Sanganeb-Atoll gesammelten Acropora-Arten kommen hier nicht weniger als 9 mit zusammen 107 Kolonien vor, aber von 10 Montipora-Arten nur 2 mit je zwei Kolonien. Das Verhältnis der Gattungsabundanz (Artenzahl $\times$ Koloniezahl) zwischen Acropora und Montipora beträgt also $963: 4$. Schon jetzt sei festgestellt, daß sich dieses Verhältnis am Testareal I mit seinem viel geringeren 
Wasseraustausch umkehrt. Dieser Befund ist geradezu charakteristisch für den Steinkorallenbewuchs in Areal IV: Gegenüber den verzweigten Wuchsformen treten die krustenförmig wachsenden Arten mit insgesamt nur 115 Kolonien auf $1,41 \mathrm{~m}^{2}$ Bodenfläche mit einem Anteil von 10,6\% aller Cnidaria und die massig wachsenden Arten mit nur 66 Kolonien auf $1,6 \mathrm{~m}^{2}$ und einem Anteil von $12,2 \%$ weit zurück.

Tab. 10. Vorkommen der 11 Acropora- und 10 Montipora-Arten in den vier Testarealen des Sanganeb-Atolls (mit Gattungsabundanz)

\begin{tabular}{|lrrrrc|}
\hline \multicolumn{1}{c}{ TQ-Nr. } & \multicolumn{1}{c}{ I } & II & III & IV & Mittelwert \\
\hline Acropora & & & & & \\
Anzahl der Arten & 3 & 5 & 6 & 9 & 5,75 \\
Anzahl der Kolonien & 6 & 24 & 25 & 107 & 40,5 \\
Gattungsabundanz & 18 & 120 & 150 & 963 & 312,75 \\
Montipora & & & & & \\
Anzahl der Arten & 8 & 8 & 4 & 2 & 5,5 \\
Anzahl der Kolonien & 26 & 33 & 17 & 2 & 19,5 \\
Gattungsabundanz & 208 & 264 & 68 & 4 & 136,0 \\
Acropora:Montipora & $18: 208$ & $120: 264$ & $150: 68$ & $963: 4$ & $312,75: 136,0$ \\
Gattungsabundanz & & & & & \\
\hline
\end{tabular}

Offensichtlich begünstigt die starke Wasserbewegung in Testareal IV die raschwüchsigen Acropora-Arten durch ihren ausgiebigen Wasseraustausch, mit dem eine reichlichere Sauerstoff- und Nahrungsversorgung gewährleistet wird, aber auch ein rascherer Abtransport des anfallenden Sediments als an den anderen Untersuchungsstellen. Vom senkrecht hängenden Areal III abgesehen, in dem sich natürlich kein Sediment ansammeln kann, weist nämlich TQIV mit nur 1,42\% Anteil am Gesamtareal die kleinsten Sand- und Feinschuttflächen auf. Dabei ist die Arealneigung mit durchschnittlich $32^{\circ}$ gegenüber der anderer Areale (I und II je $62^{\circ}$, III über $90^{\circ}$ ) weitaus geringer und sollte daher noch am ehesten Sedimentablagerungen begünstigen. Insgesamt ist der Anteil unbelebter Substratflächen (abgestorbene Korallenstöcke, Korallenfelsflächen, Grobschutt und Feinsedimente) mit $10,97 \mathrm{~m}^{2}=43,9 \%$ unter allen Arealen am geringsten und der des lebenden Bewuchses nach TQII $(57,6 \%)$ mit $55,2 \%$ am zweithöchsten. Beide Werte zusammengenommen sprechen dafür, daß in TQIV die ökologischen Bedingungen für riffbildende Cnidaria besser sind als in den anderen Arealen. Entsprechend ist die Diversität riffbildender Hydro- und Hexakorallen hoch, die der Oktokorallen hingegen sehr gering (Tab. 7).

Auch Leitarten sind nur unter den verzweigten Steinkorallen zu finden: So erreichen in TQIV die 9 Arten der Gattung Acropora zusammen 47,1\% des gesamten Cnidaria-Bestandes, darunter allein $A$. superba $24,3 \%$, und Pocillopora verrucosa fast $16 \%$. Diese beiden Steinkorallen treten als Leitarten klar hervor und spielen auch an anderen Riffabschnitten in der Umgebung von Port Sudan mit ähnlich intensiver, aber nur selten zerstörender Wasserbewegung eine bedeutende Rolle. So dominieren $A$. superba in 8 bis $12 \mathrm{~m}$ Tiefe am North Towartit-Barriereriff und $P$. verrucosa am Küsten- 
saumriff unmittelbar nördlich des Hafens, wo gerade diese Art die Struktur der Riffkante über weite Strecken hinweg bestimmt.

Auch auf Entstehung und Umfang biophysiographischer Zonen übt die Intensität der Wasserbewegung entscheidenden Einfluß aus. Natürlich charakterisieren in TestarealIV die beiden dominierenden Leitarten Acropora superba und Pocillopora verrucosa auch eine entsprechende biophysiographische Zone. Jedoch müssen zusätzlich noch die beiden Acropora-Arten corymbosa und hemprichi mit einbezogen werden, um eine klar umgrenzte Acropora-Pocillopora verrucosa-Zone (Abb. 6c, 10 und 15) mit nunmehr zusammen $56 \%$ Anteil am Gesamtbestand der Cnidaria zu erhalten.

Der hohe Anteil von Pocillopora verrucosa kennzeichnet stark brandungsexponierte Riffe ("rough water type" - Pichon, 1978b). Im sudanesischen Küstenbereich tritt diese Zone nur an besonders weit vorgelagerten Riffen (Sanganeb-Atoll, North TowartitBarriereriff) auf, nach Beobachtungen von Mergner aus den Jahren 1975 und 1979 aber auch an vorspringenden Abschnitten des Küstensaumriffs nördlich der Hafeneinfahrt von Port Sudan. Von Head (1980), der weniger exponierte, küstennahe Riffe (vor allem das Harvey-Riff) untersucht hatte, wurde diese Zone nicht angetroffen. Nur in den rein ozeanischen Riffen der pazifischen Atolle findet sich eine Zone noch höherer Brandungsbelastung, hier werden Korallen durch einen Kalkalgenrücken ("algal ridge", hauptsächlich aus Porolithon) ersetzt.

Schließlich ist noch die Frage nach typischen Indikatorarten für bestimmte Wasserbewegungsfaktoren zu stellen. Wie schon erwähnt, können die geweihartigen Kolonien einiger Acropora-Arten, etwa von $A$. hemprichi und $A$. superba, als Indikatoren für einen ausgiebigen Wasseraustausch ohne zerstörerische Brandungsdrucke an exponierten Stellen des Riffhanges in 5 bis $15 \mathrm{~m}$ Tiefe gelten. Tatsächlich herrschen in diesen Riffregionen nur selten ruhige Pendelbewegungen, aber auch keine verheerenden Brandungsbrecher. Demselben Wuchstyp ("staghorn-corals') gehören Acropora formosa und $A$. cervicornis an, die im Pazifik bzw. in der Karibik die entsprechenden strömungsexponierten Riffzonen beherrschen. Im Gegensatz zum Roten Meer können dort jedoch die "staghorn"-Bestände durch gelegentliche schwere Wirbelstürme zerstört werden (Stoddart, 1963; Maragos et al., 1973; Woodley et al., 1981).

Während also geweihförmig wachsende Acropora-Arten oft auf eine bestimmte Intensität der Wasserbewegung schließen lassen, sind als Indikatoren der Wasserbewegungsrichtung vor allem die fächerförmigen Kolonien von Millepora dichotoma bekannt geworden (u. a. Mergner, 1967, 1971, 1977; Mergner \& Schuhmacher, 1974). Stets stehen sie mit ihrer Breitseite gegen die Hauptwasserbewegungsrichtung, unabhängig davon, ob diese von Rifflängsströmungen, auftreffenden Windwellen oder dem Brandungsrücklauf bestimmt wird. Daher zeigen sie auch an Tagen mit ruhiger Wasserbewegung deren Ausrichtung an. So bilden in Testareal IV Kolonien von Millepora dichotoma und mit Einschränkung auch von $M$. platyphylla eine mehrfach gestaffelte Barriere schräg durch die Testfläche und genau rechtwinkelig zur Nordnordostrichtung, aus der die Hauptwasserbewegung auftrifft (Abb. 10).

In der Diskussion wurde zunächst die überragende Bedeutung der Wasserbewegung für die einzelnen Aspekte der Besiedlungsstruktur erkannt und am Beispiel des Testareals IV präzisiert. Damit lassen sich nun Vergleiche zu den entsprechenden Verhältnissen in den übrigen Testquadraten ziehen, zunächst zum anderen "Steinkorallenareal" II und anschließend zu den beiden "Weichkorallenarealen" I und III. 
War Testareal IV auf dem östlichen Außenriff des Atolls am stärksten gegen die Hauptrichtung der Wasserbewegung aus NNO exponiert, so ist ihr Testareal II weit weniger ausgesetzt. Zwar ist seine Fläche auf dem südwestlichen Innenriff ebenfalls gegen Nordosten gerichtet, aber wegen der kurzen Anlaufstrecke innerhalb der Atollagune nur Windwellen mittlerer Höhe ausgesetzt. So betrug die Seegangshöhe während der Untersuchungszeit 1,05 $\mathrm{m}$ und dürfte in den Sommermonaten bei etwa der Hälfte liegen. Da das TQ II nicht so tief liegt wie TQ IV, unterliegt es im oberen Bereich teilweise noch einem vertikalen Wasseraustausch, obwohl Brandung und Brandungsrücklauf geringer als am TQ IV sind. Hinzu kommt eine kräftige Längsströmung, die durch die tiefe Westpassage des Atolls hindurch am Testareal entlang nach Südosten zieht. Diese Strömungssituation ist wiederum durch verzweigte Acropora-Kolonien charakterisiert, die sich bezeichnenderweise alle im obersten Bereich des Testquadrates befinden: 5 Acropora-Arten mit 7,4\% Anteil an der Cnidaria-Fläche, darunter allein 17 Kolonien von $A$. hemprichi mit 5,5\%. Daneben und darunter anschließend herrscht jedoch Lobophyllia corymbosa mit 82 Kolonien und $39 \%$ Anteil an der Cnidaria-Fläche vor (Abb. 8,11c und 13). Diese Art kennzeichnet Standorte mit meist kräftiger Längsströmung, wie seit Crossland (1938) und Mergner (1984) von den monospezifischen Lobophyllia corymbosa-Beständen ("harbour reefs") im Uferkanal zwischen der Biologischen Station von Al-Ghardaqa und den vorgelagerten Plattformriffen bekannt ist. In der unteren Hälfte des Sanganeb-Areals II überwiegen die drei Porites-Arten Iutea, solida und sp. mit zusammen 80 Kolonien und 12,64\% Anteil am Cnidaria-Bewuchs. Dazu kommen innerhalb des Areals 33 Millepora dichotoma- und $M$. platyphylla-Kolonien mit zusammen $2,84 \%$ Anteil.

Im Gegensatz zu TestarealIV, wo verzweigte Arten das beherrschende Element unter den riffbildenden Cnidariern darstellen, machen sie in TQ II mit 96 Kolonien (von 483) auf $2,55 \mathrm{~m}^{2}$ Bodenfläche (von $12,54 \mathrm{~m}^{2}$ ) und $18,1 \%$ Anteil nur etwa ein Fünftel der $87 \%$ Riffbildner aus. Das weist auf einen schon herabgesetzten Vertikalaustausch der Wasserbewegung hin. Andererseits herrschen massig wachsende Arten vor, vor allem die Leitarten Lobophyllia corymbosa und Porites mit zusammen 293 Kolonien auf $8,59 \mathrm{~m}^{2}$ und einem Anteil von $60,7 \%$. Sie wiederum belegen die Bedeutung der kräftigen Längsströmung für den horizontalen Wasseraustausch. Das wird auch durch die stets senkrechte Ausrichtung der Millepora dichotoma-Fächer unterstrichen, die auch hier ein guter Indikator für die Hauptrichtung der Wasserbewegung sind (Abb. 11d). Den weit überwiegenden riffbildenden Cnidaria stehen, ähnlich wie in TQ IV, sehr viel weniger Nichtriffbildner gegenüber, nämlich nur 169 Kolonien auf $1,62 \mathrm{~m}^{2}$ Fläche, was $11,5 \%$ Anteil an der Cnidaria-Fläche entspricht. Davon spielt lediglich Xenia macrospiculata mit 127 Kolonien auf $1,2 \mathrm{~m}^{2}$ und mit $8,5 \%$ Anteil im unteren Bereich des Areals eine gewisse Rolle. Bezeichnend für den herabgesetzten vertikalen Wasseraustausch und seinen Ersatz durch horizontale Komponenten scheint auch das völlig umgekehrte Verhältnis zwischen Acropora und Montipora zu sein (Tab. 10), das sich mit 120:264 schon dem in TQ I annähert.

Im Verhältnis von Lebendbewuchs zu totem Substrat erweist sich Testareal II als ähnlich günstig wie TQ IV: 57,6\% der Fläche sind mit lebenden Kalkrotalgen, Poriferen, Cnidariern und Muscheln bewachsen, während 45,1\% unbelebten Substraten angehören. Vor allem in der unteren Arealhälfte machen toter Korallenfels und Grobschutt mit nur $38,83 \%$ den geringsten Anteil von allen Arealen aus. Erst an der 
Arealbasis sind mit 6,3\% Anteil auch Feinschutt und Sandflächen zu finden. Diese Befunde für Bewuchs und Substrat machen deutlich, daß auch von einer leicht herabgesetzten vertikalen, aber kräftigen horizontalen Wasserbewegung ausgehende Einflüsse für den Steinkorallenbewuchs immer noch günstig sind, nicht zuletzt auch für sein Dominieren über die nicht-riffbildenden Weichkorallen.

Diese Feststellung wird auch durch die zugehörigen Diversitätswerte belegt, die für Steinkorallen und Weichkorallen ähnlich weit auseinanderliegen wie bei TQ IV. Das verdeutlicht einerseits die Vergleichbarkeit der Lebensbedingungen in den Testarealen II und IV, andererseits den geringen Stellenwert der Weichkorallenbesiedlung in beiden Arealen gegenüber dem der Steinkorallen.

Auf die überragende Stellung von Lobophyllia corymbosa und einigen PoritesArten wurde bereits hingewiesen. Sie zeigt sich in deren Auftreten als Leitarten entsprechender biophysiographischer Zonen und, zumindest bei Lobophyllia, als Indikator für vorherrschende starke Längsströmung. Insgesamt erweist sich Testareal II im Vergleich mit TQ IV bei ausgiebigem Wasseraustausch als nahezu ähnlich günstig für riffbildende Steinkorallen, wenn auch deren Wuchsformen, Leitarten und Zonenbildner infolge der differierenden Wasserbewegungsformen andere als in TQ IV sind.

Deutlich abweichende Wasserbewegungsverhältnisse werden an den beiden "Weichkorallenarealen" I und III angetroffen, deren Front von der Hauptrichtung aus Nordnordost abgewandt und gegen Südsüdwest gerichtet ist:

So treten im T e st a re a I I nur gelegentlich hohe Windwellen aus Südwesten und Brandungsbrecher mit starkem Rücklauf auf; meist überwiegt Rifflängsströmung nach Südost bis Süd. Auch im Untersuchungszeitraum war der Seegang mit $0,65 \mathrm{~m}$ Höhe schwächer als an den übrigen Testarealen, und gelegentlich erwies sich die Südwestecke des Sanganeb-Atolls als der Bereich mit der ruhigsten Wasserbewegung. Das wird schon aus dem fast völligen Fehlen von Indikatorarten für vertikale Wasserbewegungsformen (wie Brandung und Brandungsrücklauf) offensichtlich: So bedeuten insgesamt 6 Kolonien von nur 3 Acropora-Arten für ein 25- $\mathrm{m}^{2}$-Testareal das Minimum, und selbst die im Brandungsbereich sonst weithin verbreitete $A$. hemprichi ist nur mit einer einzigen Kolonie vertreten.

Insgesamt bedecken in TQ I riffbildende Steinkorallen mit 322 Kolonien auf $5.29 \mathrm{~m}^{2}$ und einem Anteil von 50,4\% nur noch reichlich die Hälfte der Cnidaria-Siedlungsfläche, während Weichkorallen mit 484 Kolonien auf 5,19 $\mathrm{m}^{2}$ Fläche und mit $49,4 \%$ Anteil fast ebenso stark vertreten sind. Die Bestände riffbildender und nicht-riffbildender Arten sind also in TQ I ausgeglichen. Unter den Riffbildnern finden sich wiederum, wie schon in TQ II, mehr massig wachsende als verzweigte Scleractinia, nämlich 210 Kolonien der massigen Wuchsform auf $2,83 \mathrm{~m}^{2}$ mit einem Anteil von $27 \%$ gegenüber 68 Kolonien der verzweigten Arten auf $1,92 \mathrm{~m}^{2}$ mit $18,8 \%$ Anteil.

Dazu fehlen wichtige Riffbildner aus Bereichen mit kräftiger Wasserbewegung völlig, wie etwa Pocillopora verrucosa, oder weitgehend, wie Millepora dichotoma. Dagegen kommt ein sicherer Anzeiger für Bereiche ruhiger Wasserbewegung, die zartgliedrig verzweigte Seriatopora, allein in diesem Testquadrat mit zwei Drittel aller in den vier Arealen festgestellten Kolonien vor. Auch andere, gleichermaßen ausgerichtete Indikatorarten sind in TQ I zahlreich vertreten: So stellen die beiden Xenia-Arten macrospiculata und umbellata zusammen mehr als die Hälfte aller Weichkorallen und bedecken $4 / 5$ ihrer Grundfläche (Abb. 7, 11a, 12). X. macrospiculata bildet zugleich die 
hauptsächliche Leitart des Areals mit 216 Kolonien auf $3,9 \mathrm{~m}^{2}$ Fläche und einem Anteil von $37,1 \%$ der Cnidaria-Fläche. Alle diese Befunde weisen auf eine gegenüber TQ IV und II geringere hydrodynamische Beanspruchung in Testareal I hin.

Auch im Verhältnis zwischen der Häufigkeit von Acropora und der von Montipora hat sich mit $18: 208$ gegenüber den beiden anderen Arealen eine weitere Verschiebung ergeben (Tab. 10). Ein zusätzlicher Hinweis auf die veränderten Lebensbedingungen infolge der spezifischen Wasserbewegungsverhältnisse in Testareal I ist das ungünstigere Verhältnis zwischen lebender Bedeckung und unbelebtem Substrat: Obwohl die Zahl der Kolonien und lebenden Bewuchsflächen mit 972 unter allen Arealen am höchsten ist (darunter auch die Cnidaria mit 847 Kolonien), ist ihr Anteil mit $11,72 \mathrm{~m}^{2}$ und knapp $47 \%$ der Gesamtfläche doch der geringste. Das bedeutet durchschnittlich kleinere Einzelflächen pro Kolonie, offensichtlich als Folge verschlechterter Lebensbedingungen. Als Ursachen werden verringerter Wasseraustausch, Sedimentationsbelastung und auch Überwachsen durch Xenia-Kolonien angesehen. Dementsprechend größer ist der Anteil des unbelebten Substrates: Mit 13,33 $\mathrm{m}^{2}$ und 53,33\% übertrifft er den aller anderen Areale. An den toten Flächen sind Korallenfels und Grobschutt mit $45,73 \%$ sowie Feinschutt und Sand mit 7,6\% beteiligt. Beide Anteile stellen wiederum Höchstwerte dar.

Die große Anzahl von Kolonien (847) und Arten (68) in Testareal I sollte für die Cnidaria insgesamt eine hohe Diversität ergeben (Tab. 7). Tatsächlich ist der Index $\mathrm{H}^{\prime}$ mit 3,19 auch der höchste unter allen Arealen. Vielleicht wird hiervon sogar die Bildung biophysiographischer Zonen beeinflußt; denn trotz eines umfangreichen Bestandes von 216 Kolonien auf $3,9 \mathrm{~m}^{2}$ und eines Anteils von immerhin $37,1 \%$ an der Siedlungsfläche der Cnidaria formiert Xenia macrospiculata doch keine klar umgrenzte und in sich homogene Zone (Abb. 12).

Auch am zweiten "Weichkorallenareal", Testareal III, erscheint der Wasseraustausch insgesamt reduziert. Zwar laufen bei hohem Seegang von der Riffaußenseite her größere Windwellen über das Riffdach und erzeugen am Innenriff oberhalb des Testquadrats vertikale Pendelbewegungen. Doch liegt dieses mit 9,25 m mittlerer Tiefe so weit darunter, daß nur seine obersten Teile von der verstärkten Wasserbewegung profitieren. Tatsächlich finden sich dort auch nahezu alle Millepora dichotoma-Fächer und die meisten der 25 Acropora-Kolonien von 6 Arten, darunter vor allem sämtliche 8 A. hemprichi-Kolonien (auf $0,97 \mathrm{~m}^{2}$ und fast $8 \%$ der Cnidaria-Siedlungsfläche). Beide Arten können stets als Indikator kräftiger vertikaler Wasserbewegung dienen, Millepora außerdem noch deren Hauptrichtung anzeigen.

Noch klarer als in Testareal I dominieren in TQ III die Weichkorallen (Abb. 9 und 14). Dies spricht für das Vorherrschen meist ruhiger Strömungsbewegung. Während hier von den riffbildenden Cnidaria, in erster Linie den Scleractinia, nur 207 Kolonien auf $4,3 \mathrm{~m}^{2}$ mit einem Anteil von $35,0 \%$ an der Siedlungsfläche aller Cnidaria festgestellt werden können, sind es bei den nichtriffbildenden Arten, zumeist Alcyonaria, 323 Kolonien auf fast $8 \mathrm{~m}^{2}$ mit nahezu $65 \%$ Anteil, also knapp doppelt so viele. Allein die wichtigste Leitart, die Alcyonarie Sinularia polydactyla, nimmt mit 123 Kolonien auf $4,1 \mathrm{~m}^{2}$ und $33 \%$ Anteil die Hälfte der von Weichkorallen bedeckten Fläche ein. Zusammen mit den beiden anderen häufigen Arten S. leptoclados und $S$. notanda besiedelt sie sogar $44 \%$ (Abb. 11e). Gemeinsam mit der zweiten Leitart, Dendronephthya hemprichi, stellen diese vier Arten insgesamt 200 Kolonien auf $6,7 \mathrm{~m}^{2}$ und einen Anteil von fast 
$54 \%$ aller Cnidaria. Weichkorallen erreichen in diesem Areal ihre höchste Diversität von allen (Tab. 7).

Das Auftreten von Dendronephthya hemprichi an einem seichten, voll belichteten Standort ist ungewöhnlich. Die azooxanthellaten (zooxanthellenfreien) Dendronephthya-Arten finden sich gewöhnlich in Schattenbereichen, in geringer Tiefe unter Überhängen und im tieferen Vorriff auch freistehend. Der in TQ III vorgefundene Dendronephthya-Bestand dürfte demnach einen metastabilen Zustand darstellen. Unabhängig von seiner Entstehung verdankt er seinen Erhalt höchstwahrscheinlich den vergleichsweise guten Emährungsbedingungen an diesem Standort. Dieser erlaubt nämlich den ausschlieBlich heterotrophen Kolonien das Plankton aufzufangen, das nachts vom Riffdach aufsteigt und herangeschwemmt wird. Die Kolonien, die verzweigten Schläuchen ähneln, ragen dann weit in den freien Wasserraum hinaus. Tagsüber hängen sie hingegen schlaff herab, schatten den Korallenfelsuntergrund ab und verhindern das Aufwachsen von zooxanthellaten Raumkonkurrenten.

Unter den Steinkorallen sind die Wuchsformen ziemlich gleichmäßig verteilt: So treffen auf verzweigte Arten 48 Kolonien auf $1,6 \mathrm{~m}^{2}$ Fläche mit einem Anteil von etwa $13 \%$ an der Cnidaria-Fläche, auf krustenförmige Arten 60 Kolonien mit $1,27 \mathrm{~m}^{2}$ Fläche und 10,3\% Anteil und auf massig wachsende Arten 99 Kolonien mit 1,4 $\mathrm{m}^{2}$ Fläche und $10,4 \%$ Anteil. Auch das Verhältnis Acropora zu Montipora (Tab. 10) ist mit 150:68 ausgeglichener als in den anderen Testarealen, vor allem in I und IV, und daher nicht so aussagekräftig.

Das gleiche gilt für das Verhältnis von lebenden Bewuchsflächen zu unbelebtem Substrat: Lebendbewuchs bedeckt mit 764 Einzelflächen und Kolonien, darunter nur 530 der Cnidaria, aber 149 der Porifera (beide Zahlen stellen Extremwerte dar), rund $13 \mathrm{~m}^{2}$ und damit einen Anteil von $52,2 \%$. Ihnen stehen an unbelebtem Substrat $11,3 \mathrm{~m}^{2}$ mit einem Anteil von $45,3 \%$ gegenüber. Diese Fläche besteht ausschließlich aus Korallenfels, da sich in der teilweise überhängenden Wand Schutt und Sand natürlich nicht ansammeln können.

Faßt man die Ergebnisse noch einmal zusammen, so erscheinen die Innen- und Außenriffe auf den ersten Blick bemerkenswert ähnlich: Die Cnidaria-Bedeckung schwankt nur zwischen 42,4 und $56,6 \%$, und die Diversitätswerte liegen zwischen $\mathrm{H}^{\prime}=2,34$ und 2,77 beieinander. Die detaillierte quantitative Analyse ermöglicht jedoch eine genauere Auflösung dieses zunächst groben und daher unscharfen Bildes.

Differenziert in Hydro-, Stein- und Weichkorallen, ergeben sich nämlich aufschlußreiche Unterschiede: Bedeckungsrate und Diversität der hermatypischen (riffbildenden) Formen sind auf den hydrodynamisch exponierten Riffseiten deutlich größer als auf den geschützten Seiten. Dieser Befund stimmt mit dem Ergebnis der ComputerSimulation des Wirkungsspektrums abiotischer Faktoren in einem idealisierten Riff (Chappell, 1980) überein. Gleichzeitig bestätigen das zahlenmäßige Dominieren und die größere Diversität der Weichkorallen in hydrodynamisch geschützten Riffpartien die entsprechenden Aussagen von Schuhmacher (1975) und Dinesen (1983). Zu den wenigen Arbeiten zur Riffbesiedlung, die neben Steinkorallen auch Weichkorallen berücksichtigen, gehört die Untersuchung von 8 Vorriffabschnitten an der Nordwestküste des Golfes von Aqaba (Benayahu \& Loya, 1977). Die Bedeckungsraten von Steinund Weichkorallen wie auch das zugrunde liegende hydrodynamische Regime sind dort nicht so unterschiedlich ausgeprägt wie am Sanganeb-Atoll, weswegen wohl auch 
der ursächliche Zusammenhang beider Erscheinungen in dieser Arbeit nicht zum Ausdruck kommt.

Die im wesentlichen hydrodynamisch begründete unterschiedliche Besiedlung durch Stein- und Weichkorallen zieht letztlich noch weitergehende Folgerungen nach sich: Da zum Riffwachstum definitionsgemäß nur hermatypische Formen beitragen, folgt hieraus, daß sich der Riffring des Sanganeb-Atolls gegen die vorherrschende Windrichtung verbreitern sollte. Diese Schlußfolgerung steht im Gegensatz zu Angaben von Davies \& Kinsey (1977), die am One Tree-Reef (ein atollähnliches Plattformriff im südlichen Großen Barriereriff) eine Ansammlung von Kalk vornehmlich auf der Leeseite, vor allem in der Lagune, festgestellt haben. Welchen Anteil hierbei Sedimente, die über das Riff gespült werden, haben, bleibt an beiden Orten noch zu untersuchen. Eine generalisierende Aussage zum bevorzugten lateralen Wachstum ozeanischer Riffe scheint demnach noch nicht möglich.

Die vorgelegte quantitative Auswertung der Testquadrate ist bei weitem nicht erschöpfend; sie soll vielmehr zunächst die charakteristischen Grundzüge der Besiedlungsgemeinschaften an den vier Riffhängen des einzigen Atolls im Roten Meer darstellen. Weitere Analysen des Karten- und Tabellenmaterials zu speziellen Aspekten der Besiedlungsstrukturen - insbesondere eine Vertiefung der statistischen Auswertung und deren Diskussion vor dem Hintergrund der vorliegenden biophysiographischen Erfassung - werden noch folgen. Darüber hinaus kann das vorgelegte Material als Grundlage für populationsdynamische Untersuchungen über die Zeit hinweg dienen. Schließlich sind die mit der Quadratmethode gewonnenen Daten sehr gut für den Vergleich von Korallengemeinschaften verschiedener geographischer und topographischer Lagen geeignet. Dafür wird im II. Teil der Gesamtarbeit (Schuhmacher \& Mergner, 1985) zum ersten Mal ein Beispiel vorgelegt.

Danksagungen. Wir danken der Deutschen Forschungsgemeinschaft für die finanzielle Unterstützung unseres Sanganeb-Projektes, unseren beiden Mitarbeitern cand. rer. nat. F. Nobbe und L. Schillak (Ruhr-Universität Bochum) für ihren unermüdlichen Einsatz bei den Vor- und Nachbereitungen der Forschungsreise, bei der Erfassung der ökologischen Daten, beim Aufsammeln der Belegstücke und bei der täglichen Wartung und Instandhaltung des Gerätes sowie den sudanesischen Dienststellen für die Förderung unserer Untersuchungen, insbesondere dem National Council for Research and Technical Development für die Genehmigung des Forschungsprojektes und der erforderlichen Aufsammlungen, und dem Survey Department, Khartoum, für die Abgabe einer Anzahl von Luftbildern der interessierenden Riffregion und die Erlaubnis zu ihrer Veröffentlichung. Besonderer Dank gebührt dem Direktor des Institute of Oceanography Port Sudan, Professor Dr. J. Schroeder, und den Institutsangehörigen für ihre Hilfe bei den Zollformalitäten, bei der praktischen Durchführung des Projektes und der Ausstattung mit zusätzlich erforderlichem Gerät sowie der Besatzung des Sanganeb-Leuchtturmes für ihre freundliche Aufnahme und Gastlichkeit. Bei der Auswertung der Untersuchungsergebnisse haben in dankenswerter Weise vor allem Herr H. Löseke durch Erfassen des Datenmaterials in Tabellenform, cand. rer. nat. B. Dittrich durch Berechnen der Diversitätsindices und Herr J. Rawlinson durch Herstellen der Photounterlagen mitgewirkt. Unser herzlicher Dank gilt Herrn F. Theissen für die mit großer Sorgfalt und außerordentlichem Einfühlungsvermögen angefertigten Schwarzweiß-Zeichnungen der Besiedlungsstruktur und Farbpläne der Artenvielfalt in den Testarealen. Schließlich möchten wir den Kollegen unseren besonderen Dank aussprechen, die taxonomisch schwierige Belegexemplare bestimmten bzw, unsere Determinationen überprüften: Dr. J, Verseveldt (Alcyonaria), Dr. G. Scheer und Dr. M. Wijsman-Best (Scleractinia). Die Herstellung der Farbdrucke wurde aus Titel 54794, RUB, finanziert. 


\section{LITERATUR}

Barnes, D. J. \& Taylor, D. L., 1973. In situ studies of calcification and photosynthetic carbon fixation in the coral Montastrea annularis. - Helgoländer wiss. Meeresunters. 24, 284-291.

Barnes, J., Bellamy, D. J., Jones, D. J., Whitton, B. A., Drew, E. A., Kenyon, L., Lythgoe, J. N. \& Rosen, B. R., 1971. Morphology and ecology of the reef front of Aldabra. -Symp. zool. Soc. Lond. $28,87-114$.

Benayahu, Y. \& Loya, Y, 1977. Space partitioning by stony corals, soft corals and benthic algae on the coral reefs of the northern Gulf of Eilat (Red Sea). - Helgoländer wiss. Meeresunters. 30, 362-382.

Bohnsack, J, A, 1979. Photographic quantitative sampling of hard-bottom benthic communities, Bull. mar. Sci. 29, 241-252.

Bouchon, C., 1981. Quantitative study of the scleractinian coral communities of a fringing reef of Réunion Island. - Mar. Ecol. Prog. Ser. 4, 273-288.

Bradbury, R. H. \& Young, P. C., 1981. The effects of a major forcing function, wave energy, on a coral reef ecosystem. - Mar. Ecol. Prog. Ser. 5, 229-241.

Braithwaite, C. J. R., 1982. Patterns of accretion of reefs in the Sudanese Red Sea.-Mar. Geol. 46, $297-325$.

Chappell, J., 1980. Coral morphology, diversity and reef growth. - Nature, Lond. 286,249-252.

Cloud, P. E., 1957. Nature and origin of atolls. - Proc. 8th Pacif. Sci. Congr. 3a, 1009-1036.

Crossland, C., 1907. Reports on the marine biology of the Sudanese Red Sea. IV. The recent history of the coral reefs of the mid-west shores of the Red Sea. - J. Linn. Soc. 31, 14-30.

Crossland, C., 1911. Reports on the marine biology of the Sudanese Red Sea. XVIII. A physical description of Khor Dongonab, Red Sea. - J. Linn. Soc. 31, 265-286.

Crossland, C., 1913. Desert and water gardens of the Red Sea. Univ. Press Cambridge, $158 \mathrm{pp}$.

Dahl, A. L., 1973. Surface area in ecological analysis: quantification of benthic coral-reef algae. Mar. Biol. 23, 239-249.

Darwin, C., 1842. The structure and distribution of coral reefs. Smith, Elder \& Co., London, $214 \mathrm{pp}$.

Davies, P. J. \& Kinsey, D. W., 1977. Holocene reef growth - One Tree Island, Great Barrier Reef. Mar. Geol. 24, M1-M11.

Dinesen, Z. D., 1983. Patterns in the distribution of soft corals across the central Great Barrier Reef. Coral Reefs $1,229-236$.

Girdler, R. W., 1969. The Red Sea - a geophysical background. In: Hot brines and recent heavy metal deposits in the Red Sea. Ed. by E. T. Degens \& D. A. Ross. Springer, Heidelberg, 38-58.

Gohar, H. A. F., 1954. The place of the Red Sea between the Indian Ocean and the Mediterranean. Hidrobiol. Publ. Fak. Sci. Univ. Istanbul (B) 2, 47-82.

Handbuch für das Rote Meer und den Golf von Aden, 1963. Deutsches Hydrographisches Institut, Hamburg, 2034, 1-569.

Head, S. M., 1980. The ecology of corals in the Sudanese Red Sea. Thesis, Cambridge Univ., $471 \mathrm{pp}$.

Hiatt, R. W., 1957. Factors affecting the distribution of corals on the reefs of Arno Atoll, Marshall Islands. - Proc. 8th Pacif. Sci. Congr. 3 A, $929-970$.

Klunzinger, C. B., 1879. Die Korallthiere des Rothen Meeres. 2: Die Steinkorallen. 1. Die Madreporaceen und Oculinaceen. Gutmann, Berlin, $88 \mathrm{pp}$.

Krenkel, E., 1925. Geologie Afrikas. 1: Geologie und Bodenschätze Afrikas. Borntraeger, Berlin, $471 \mathrm{pp}$.

Kühlmann, D. H. H., 1983, Composition and ecology of deep-water coral associations. - Helgoländer Meeresunters. 36, 183-204.

Littler, M. M., 1971. Standing stock measurements of crustose coralline algae (Rhodophyta) and other saxicolous organisms. $-J$, exp. mar. Biol. Ecol. 6, 91-99.

Maragos, J. E., Baines, G. B. K. \& Beveridge, P. J., 1973. Tropical cyclone Bebe creates a new formation on Funafuti Atoll, - Science, N. Y. 181, 1161-1164.

Marshall, P. 1931. Coral reefs - rough water and calm water types. - Rep. Gt Barrier Reef Comm. 8 , $64-72$.

McNeil, F. S., 1972. Physical and biological aspects of atolls in the northern Marshalls. - Proc. Symp. Corals and Coral Reefs $1969,507-567$. 
Mergner, H., 1967. Über den Hydroidenbewuchs einiger Korallenriffe des Roten Meeres. $-\mathrm{Z}$. Morph. Okol. Tiere 60, 35-104.

Mergner, H., 1971. Structure, ecology and zonation of Red Sea coral reefs (in comparison with South Indian and Jamaican reefs). - Symp. zool. Soc. Lond. 28, 141-161.

Mergner, $H ., 1977$. Hydroids as indicator species for ecological parameters in Caribbean and Red Sea coral reefs. - Proc. 3rd int. Coral Reef Symp., Miami 1, 119-125.

Mergner, H., 1979. Quantitative ökologische Analyse eines Rifflagunenareals bei Aqaba (Golf von Aqaba, Rotes Meer). - Helgoländer wiss. Meeresunters. 32, 476-507.

Mergner, H., 1984. The ecological research on coral reefs of the Red Sea. - Deep Sea Res. (A) 31, 855-884.

Mergner, H., 1985. Initial recolonization of Funafuti Atoll coral reefs devastated by hurricane "Bebe". - Atoll Res. Bull. 284, 1-30.

Mergner, H. \& Schuhmacher, H., 1974. Morphologie, Okologie und Zonierung von Korallenriffen bei Aqaba (Golf von Aqqaba, Rotes Meer). - Helgoländer wiss. Meeresunters. 26, 238-358.

Mergner, H. \& Schuhmacher, H. 1981. Quantitative Analyse der Korallenbesiedlung eines Vorriffareals bei Aqaba (Rotes Meer). - Helgoländer Meeresunters. 34, 337-354.

Mergner, H. \& Svoboda, A., 1977. Productivity and seasonal changes in selected reef areas in the Gulf of Aqaba (Red Sea). - Helgoländer wiss. Meeresunters. 30, 383-399.

Molengraaf, G. A. F., 1930. The coral reefs in the East Indian Archipelago, their distribution and mode of development. - Proc. 4th Pacif. Sci. Congr. 2a, 55-89.

Nawab, Z. A., 1984. Red Sea mining: A new era. - Deep-Sea Res. (A) 31, 813-822.

Pichon, M., 1978a. Problems of measuring and mapping coral reef colonies. In: Coral reefs: Research methods. Ed. by D. R. Stoddart \& R. E. Johannes. Unesco, Paris, 219-230.

Pichon, M., 1978b. Recherches sur les peuplements à dominance d'anthozoaires dans les récifs coralliens de Tuléar (Madagascar). - Atoll Res. Bull. 222, 1-447.

Pielou, E. C., 1966. The measurement of diversity in different types of biological collections. J. theor. Biol. 13, 131-144.

Purdy, E. G., 1974. Reef configurations: Cause and effect. - SEPM Spec. Publ. 18, 9-76.

Scheer, G., 1964. Bemerkenswerte Korallen aus dem Roten Meer. - Senckenberg. biol, 45, 612-620.

Scheer, G., 1984. The distribution of reef-corals in the Indian Ocean with a historical review of its investigation. - Deep Sea Res. (A) 31, 885-900.

Scheer, G. \& Pillai, C. S. G., 1983. Report on the stony corals from the Red Sea. - Zoologica, Stuttgart 133, $1-198$.

Schilling, J. G., 1969. Red Sea floor origin: rare-earth evidence, - Science, N. Y. 165, 1357-1360.

Schuhmacher, H., 1975. Die Rolle der Weichkorallen (Alcyonacea, Octocorallia) innerhalb der Riffbiozönosen des Roten Meeres und des australischen Großen Barriereriffs. - Verh. dt. zool. Ges. 67, 380-384.

Schuhmacher, H. \& Mergner, H., 1985. Quantitative Analyse von Korallengemeinschaften des Sanganeb-Atolls (mittleres Rotes Meer) - II. Vergleich mit einem Riffareal bei Aqaba (nördliches Rotes Meer) am Nordrande des indopazifischen Riffgürtels. - Helgoländer Meeresunters. $39,419-440$.

Stoddart, D. R., 1963. Effects of hurricane Hattie on the British Honduras reefs and cays, October 30-31, 1961. - Atoll Res. Bull. 95, 1-142.

Stoddart, D. R., 1969. Ecology and morphology of recent coral reefs. - Biol. Rev. 44, 433-498.

Svoboda, A., 1978. In situ monitoring of oxygen production and respiration in Cnidaria with and without zooxanthellae. In: Physiology and behaviour of marine organisms. Ed by D. S. McLusky \& A. J. Berry. Pergamon Press, Oxford, 75-82.

Vine, P. J. \& Vine, M. P., 1980. Ecology of Sudanese coral reefs with particular reference to reef morphology and distribution of fishes. - Proc. Symp. Coastal and Marine Environment of the Red Sea, Gulf of Aden and Tropical Western Indian Ocean, Khartoum 1, 88-140.

Weinberg, S., 1981. A comparison of coral reef survey methods. - Bijd. Dierk. 51, 199-218.

Wells, J. W., 1954. Recent corals of the Marshall Islands. - Prof. Pap. U.S. geol. Surv. 260-I, 385-486.

Wells, J. W., 1957. Coral reefs. - Mem. Geol. Soc. Am. 67, 600-631.

Wong, K. H. W. \& Degens, E. T., 1980. The Red Sea and the Gulf of Aden: a review, - Proc. Symp. Coastal and Marine Environment of the Red Sea, Gulf of Aden and Tropical Western Indian Ocean, Khartoum 3, 213-266. 
Wood-Jones, F., 1910. Coral and atolls. Reeve, London, $392 \mathrm{pp}$.

Woodley, J. D. et al., 1981. Hurricane Allen's impact on Jamaican coral reefs. - Science, N. Y. 214, $749-755$.

\section{ERRATA}

(Farbtafeln und Legenden)

In allen Legenden muß in den Farbkästchen für Porifera nur $\mathrm{P}$ (nicht $\mathrm{Po}$ ) stehen.

Die wechselnde Verwendung schwarzer oder weißer Artnummern in den Legenden und Kartenplänen je nach Untergrund ist in jedem Fall gleichrangig.

In den Legenden fehlen einzelne Artnummern, die als Artflächen in den Kartenplänen ausgewiesen, aber nicht in Tabelle 3 enthalten sind, so: in Legende zu Abb. 12 Nr. 61 (in Einzelquadrat IIIc, Mitte links); in Legende zu Abb. 13 Nr. 67 (in Ic und IVb, unten rechts); in Legende zu Abb $14 \mathrm{Nr} .117$ (in Ile, oben Mitte); in Legende zu Abb. 15 Nr. 103 (in le, unten links), Nr. 106 (in IIIa, oben Mitte).

In Kartenplänen fehlen einzelne Artflächen, die in den Legenden und in Tabelle 3 aufgeführt sind, so: auf Karte zu Abb. 13 Nr. 117; auf Karte zu Abb. 15 Nr. 124 (als Linie entlang Grenze III/IVe vorhanden, aber nicht farbig ausgeführt).

In Kartenplänen unvollständige oder falsche Darstellung einzelner Artflächen, so auf Karte zu Abb. 13: Auf Artfläche $12 \mathrm{in} \mathrm{Ib/c}$, Mitte, fehlt rote Punktierung; auf Karte zu $\mathrm{Abb}$. 15: Um Artfläche 119 in Ve, unten rechts, fehlt der dunkelviolette Rand; auf Artfläche 37 in Ib, unten links, fehlt die Artnummer 37; Artfläche 36 in IIc, Mitte links, muß die Artnummer 34 erhalten; Artflächen 4 in Ia, unten links; IIa, oben Mitte, und Va, oben links, sind blau mit schwarzer Punktierung, in der Legende jedoch mit weißer Punktierung dargestellt. 